Image Potential states at Metal-Dielectric Interfaces

\author{
by \\ Walter Richardson Merry Jr. \\ Fh.D. Thesis \\ Department of Chemistry \\ University of California \\ and \\ Chemical Sciences Division \\ Lawrence Berkeley Laboratory \\ university of California \\ Berkeley, CA 94720
}

$[B L-32352$

DE92 017019

April, 1992

This work was supported by the Director, Office of Energy Research, office of Basic Energy Sciences, Chemical Sciences Division of the U. S. Department of Energy, under Contract No. DE-ACO3-76SF00098. 
Image Potential states at Metal-Dielectric Interfaces

by

Walter Richardson Merry, Jr.

Abstract

Angle-resolved two-photon laser photoemission was used to observe the image potential electronic states on the (111) face of a silver single cystal. The transient image potential states were excited from the occupied bulk bands with photons whose energy was tunable around $4 \mathrm{eV}$. Photoemission of the image potential states was accomplished with photons of energy tunable around $2 \mathrm{eV}$. Image potential states were found to persist in the presence of physisorbed adlayers of xenon and cyclohexane. On clean Ag(111), the effective mass of the $n=1$ image potential state was found to be $1.4 \pm 0.1$ times the mass of a free electron $\left(m_{e}\right)$. A binding energy of $0.77 \mathrm{eV}$, measured by earlier workers, was assumed in analysis of the data for the clean surface. On $\mathrm{Ag}(111)$ at $75 \mathrm{~K}$ covered by one monolayer of xenon, the binding energy of the $n=1$ image potential state was unchanged relative to its value on the clean surface. An effective mass of $(1.00 \pm 0.05) \cdot m_{e}$ was obtained. On Ag(111) at $167 \mathrm{~K}$, covered by one monolayer of cyclohexane, the binding energy of the $n=2$ member of the image potential series was $0.30 \pm 0.05 \mathrm{ev}$. The energy of the $n=1$ state was again unchanged by deposition of the adsorbate. The effective masses of both states were $(0.90 \pm 0.1) \cdot m_{e}$ " The 
experimental results are compared to a model based on the multiple reflection formalism. The ultra-high vacuum chamber designed for this experiment is described extensively. The sample, held in an angle-resolving manipulator, was cooled through a flexible copper rope connected to a stationary liquid helium flow cryostat. A cavity-dumped, synchronously pumped dye laser producing pulses of 1.0 ps duration at a repetition rate of 1 or $2 \mathrm{MHz}$ was used as a tunable light source. The UV light needed for excitation of the image potential states was generated as the second harmonic of the visible laser output. 
I dedicate this work

to my parents and grandparents. 


\section{Table of contents}

Table of Figures . . . . . . . . . . . . . . . . . . v v

Introduction . . . . . . . . . . . . . . . . viii

Acknowledgements . . . . . . . . . . . . . . . . . . . xi

1. Backgrouna . . . . . . . . . . . . 1

1.1 Theory of Image Potential States... . 1

1.1.1 Classical Image Potential. . . . 1

1.1.2 Hydrogenic Model . . . . . . . 6

1.1.3 Multiple Reflection Theory . . . . 14

1.1.3.1 Introduction......... 14

1.1 .3 .2 Literature......... 15

1.1.3.3 Derivation.......... 17

1.1.3.4 Effective Mass....... . 39

1.1.4 Other Theories . . . . . . . . 44

1.1.5 Adsorbate Theories . . . . . . 47

1.2 Experiments on Image Potential States . 49

1.2.1 Inverse Photoemission . . . . . . 49

1.1 .2 Two-Photon Photoemission . . . . 53

1.3 Electronic properties of Silver (111) • 63

Experimental . . . . . . . . . . . 77 
2.1 U1tra-High Vacuum system . . . . . . 78

2.1.1 Original Chamber . . . . . . . 78

2.1 .2 New Chamber... . . . . . 80

2.1 .3 Bakeout............. 89

2.1.4 Manipulator . . . . . . . . 91

2.1 .5 Sample Holder... . . . . . . 94

2.1.6 Sample cooling . . . . . . . 107

2.2 Sample Preparation . . . . . . . . 111

2.3 Laser System . . . . . . . . . 118

2.4 Time-of-Flight Analysis . . . . . . 121

2.4 .1 Literature . . . . . . . . 122

2.4 .2 Detector . . . . . . . . 127

2.4 .3 Jacobian Effect. . . . . . 135

2.4.4 Error Analysis . . . . . . 136

2.5 Contact Potential Difference... . . 140

3 Results and Discussion . . . . . . . . 148

3.1 Clean Silver (111). . . . . . . 148

3.2 Xenon-Covered Silver(111) . . . . . 173

3.3 Cyclohexane-Covered Silver (111) • • 193

4 Conclusion . . . . . . . . . . . . 208

References . . . . . . . . . . . . . . . 212 


\title{
Table of Ficures
}

Figure:

Page:

1 Classical image potential 3

2 Energy levels of image potential 9

3 Probability densities of image potential state in hydrogenic approximetion

4 Diagram of multiple reflection of image potential state amplitude flux

\author{
3
}

9

like bands used in multiple reflection theory

6 Complex wave vector used in inultiple reflection theory

Calculation of effective masses of reflection theory

8 Bulk and surface Brillouin zones for (111) face of FCC lattice silver

10 Projected bulk band structure of silver(111)

11 Assembly view of ultra-high vacuum chamber

12 Schematic drawing of vacuum chamber showing important rotation axes

13 Detail of angle-resolved sample manipulator

14 Illustration of angle-resolved manipulator showing action of mechanism 
15 Illustration of sample holder with cooling appendage attached

16 Laue pattern of silver(111) showing orientation of surface Brillouin zone

17 Exploded view of microchannelplate assembly

Electrical schematic of two-photon photoemission experiment with timeof-flight detection

19 Contact potential diagrain for timeof-Flight detector and clean silver(111)

20 Time-of-fight distribution of twophoton photoelectrons from clean silver(111)

21 Kinetic energy distributions of twophoton photoelectrons from clean silver (111)

22 Laser wavelength survey of twophoton photoelectron excitations of clean silver(111)

23 Diagram of mechanisms whereby twophoton photoelectrons may be excited

24 Two-photon photoelectron kinetic energy distributions at a series of sample rotation angles

25 Experimentally determined dispersion of $n=1$ image potential state on silver(11.1)

Map of bujk electronic bands of silver used in multiple reflection theory calculation

Phase shifts calculated for $n=1$ image potential state on clean silver (111)

Langmuir dosing of xenon on silver(111) at $25 \mathrm{~K}$ 
29 Contact potential diagram for sample covered by physisorbed adlayer

Binding energy of $n=1$ image potential state on xenon-covered silver(111)

31

Phase shifts calculated for $n=1$ image potential state on xenoncovered silver(111)

32 Experimentally determined dispersion of $n=1$ image potential state on xenon-covered silver(11.1)

Two-photon phutoemission from silver(111) covered by one monolayer of cyclohexane

Dispersion of $n=1$ and $n=2$ image potential states on cyclohexanecovered silver(111)

Langmuir dosing of cyclohexane on silver(111)

Enlarged view of $n=1$ image potential state following exposure to 2.3 Langmuirs cyclohexane 


\section{Introduction}

The interactions of electrons with surfaces are of considerable importance in nature. Chemisorption, for exzmple, proceeds by the exchange of electrons between a surface and an adsorbed species. Physisorption, while it does not entail wholesale transfer of electrons between the surface and adsorbate, operates by the redistribution of charge within the two. In various forms of surface spectroscopy, such as electron energy loss, Auger and photoelectron spectroscopy, the experimenter gains important information on the bulk and surface properties of a material by juciciously arranging the transfer of energy to or from the electrons which are observed. The revolutionary fields of scanning tunneling microscopy and atomic force microscopy are based fundamentally upon electronic interactions at surfaces.

In the present work, the electronic properties of surfaces have been explored from the vantage point of a unique type of electronic surface state. This is the image potential state. It consists of an electron quantum-mechanically bound in the region of space just outside tile material. This region may extend from a few to several tens of Angstroms outside the surface. The electron is bound to the surface by an energy of up to about an electron volt. Thus the characteristic energy and size scale of the image potential state is that of concern to chemistry. 
The specific system of concern in the present work was a metal surface covered by a thin film of electrically nonconducting material. The behavior of image potential states has been explored in such systems with insulating films whose thicknesses have ranged from a single atom to several molecular layers. Two chief goals have been to examine the sensitivity of the image potential state to the presence of the adsorbate, and to gain novel insight into inicroscopic adsorbate-surface interactions as probed by the image potential state.

Since the interactions between image potenilal states and dielectric films on metal surfaces have been explored for the first time in this work, attention was focussed upon physisorbed systems, where chemical interactions between the adsorbate and substrate are minimized. Physisorbed systems, in fact, have proved to be complicated enough. This work has cast a spotlight upon fundamental questions concerning the physical nature of the adsorbed species and their response to and effect upon the image potential state which probes them. A central question which has emerged is whether the quantum mechanical electronic energy level structure of the adsorbed species or its continum dielectric properties affect the image potential state more strongly. The culmination of this line of inquiry should embody a synthesis of these two points of view.

In a methodological sense, this experimental study has 
drawn upon the techniques of surface physics, laser spectroscopy, and photoelectron spectroscopy. The need to perform the experiments using well-characterized metal surfaces free of contamination has made standard ultra-high vacuum techniques a necessity. substantial effort was expended meeting the demands of photoelectron energy analysis, sample characterization and attainment of sample temperatures at which physisorption of the desired adsorbates was possible. Since image potential. states are normally unoccupied and decay on ultrafast timescales, their creation and observation were carried out by laser excitation. In particular, the technique of two-photon photoemission was central to this work. The implementation of this method was optimized to a considerable degree. A detailed exposition of these aspects of the project is given in the present work. 


\section{Acknowledgements}

Having reached this bend in the stream of life, I would like to offer my thanks to many people. My parents have been a boundiess source of inspiration and support and have always gone out of their way to provide me with every opportunity for growth. It was typical of them to make certain, years ago, that I would have a chance at the best education possible.

I shall always appreciate the invaluable lessons learned from my research director, Professor Charles Harris. He has taught me a creative and original approach to science, as well as the importance of thinking big in intellectual pursuits and in life. I also appreciate his never losing faith in my ability, or at least not appearing to if he actually did. vijaya Narasimian has been a valued friend during my years at Berkeley. For me, graduate school would have been a far bumpier road without her sage advice, frank counsel and superb administrative abilities.

It was extremely rewarding to have worked closely with Robert Jordan and Dave Padowitz on the project described in this tome. Their creativity and tenacity have been invaluable, particularly when mine occasionally flagged. It was my good fortune that Robert and I were originally launched together into the project which eventually transmuted into the experiments described in this work. over the years, our experiences far outgrew the bounds of being coworkers. Although Dave began working with us at a later stage, he has 
more than compensated for this shortcoming by his expertise, insight and wide-ranging interests. These interests have ranged at least as widely as raising baby squid in the laboratory. The patience of Robert and Dave in the face of my occasional outbursts of morosity is worthy of special mention.

over the years, Eric Peterson and I have covered many miles together on our trusty bicycles. The cross-country moving expedition was aiso an experience I shail not forget. To him and Tsurumi, I extend my wishes for a lifetime of happiness. I have long cherished the company of my friends at work on our traditional 2 PM cappucino runs to Roma's. Prominent among these worthies have been Dan Russell, Jennifer Hane, Dee Pennington, Steve Gadd, Eric Haas and occasionally even Karen E. Schultz, who actually despises coffee and thus wins the "you-figure-it-out" award. More recently, the ranks of the Roma's crowd have swelled agreeably to include Jason King, Robert Lingle and Jason MCNeill. I would like to thank all. those with whon I have worked in Professor Harris' laboratory for the erudite, fun-loving and incomparable environment they have created.

Lee Stacy, an excellent friend since grade school, has long been an important person in my life. Together, we are veterans of countless canoe trips and many midnight moped rides. I would also like to mention two other old friends, Paul Arias and Don Hood, who, sadly, are no longer with us to read this. I have been lucky to have shared the infamous, 
rent-controlled apartment at 2609 Ellsworth with three mellow, fun people at different times over the years. Peter Giannousis is a great guy who knew how to keep a reasonable schedule in graduate school. Dan Mullen always had excelient taste in value-priced wines and a heck of a record collection, to boot. Steve carrier is an excellent cook, a brewer of fine coffee, and a holder of intriguing views on the subject of economics.

Many aspects of the experimental work described in this volume would have been impossible without several knowledgeable, skilled and patient people in the college of Chemistry. I have greatly appreciated the expert help of George Weber, Hans Graetsch, Ron Dal Porto, Chuck Souders and Scott Bonilla in the machine shop. Equally valuable has been the help of Henry Chari of the electronics shop and Bob Koch of the instrument repair shop, from whom I have learned much that is practical and valuable.

This work was supported by the United States Department of Energy, Office of Basic Energy Sciences, Chemical sciences Division under contract number DE-ACO3-76SF00098. 
The classical image potential is an important aspect of the electrostatics of surfaces.' As in Figure 1, a plane surface of a dielectric or a conductor is considered, with vacuum existing outside the material. When a point charge $(-q)$ is brought near the surface, the induced flow of free charge in the conductor or the polarization of fixed charge in the dielectric leads to accumulation of a distribution of surface charge whose sign is opposite that of the external point charge.

The detailed nature of the surface charge distribution is such that the electric field existing between it and the point charge in the region outside has the same form as a hypothetical field between point charge $(-q)$ and a putative point charge of opposite sign placed as a mirror image of the first behind the surface plane. The second charge is referred to as the image charge. When it is considered, the actual material giving rise to it is viewed as having been removed from the system.

While the image charge is always opposite in sign to the real, physical charge, its magnitude may be less. Only when the material is a conductor will the two charges be equal in magnitude. On surfaces of dielectrics, the image charge is 
Caption for Figure 1:

a) Charge $-q$ placed distance $z$ above the surface of the material induces an effective image charge $+\beta q$ below the surface plane. The value of $\beta$ is determined by the static dielectric constant of the material.

b) The potential energy due to image charge formation depends only upon $z$ and not upon displacement parallel to the surface plane. The potential is attractive for all materials. 
a)

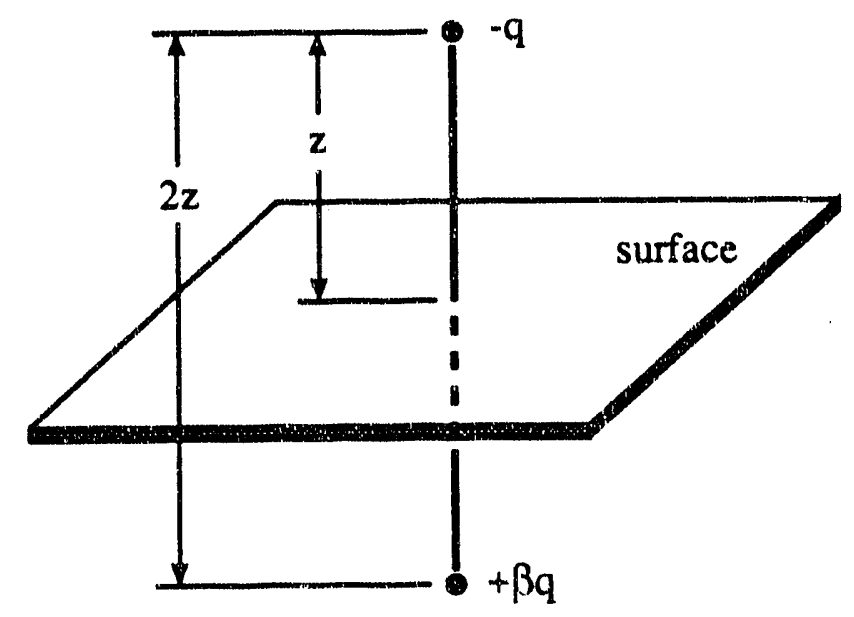

b) potential energy

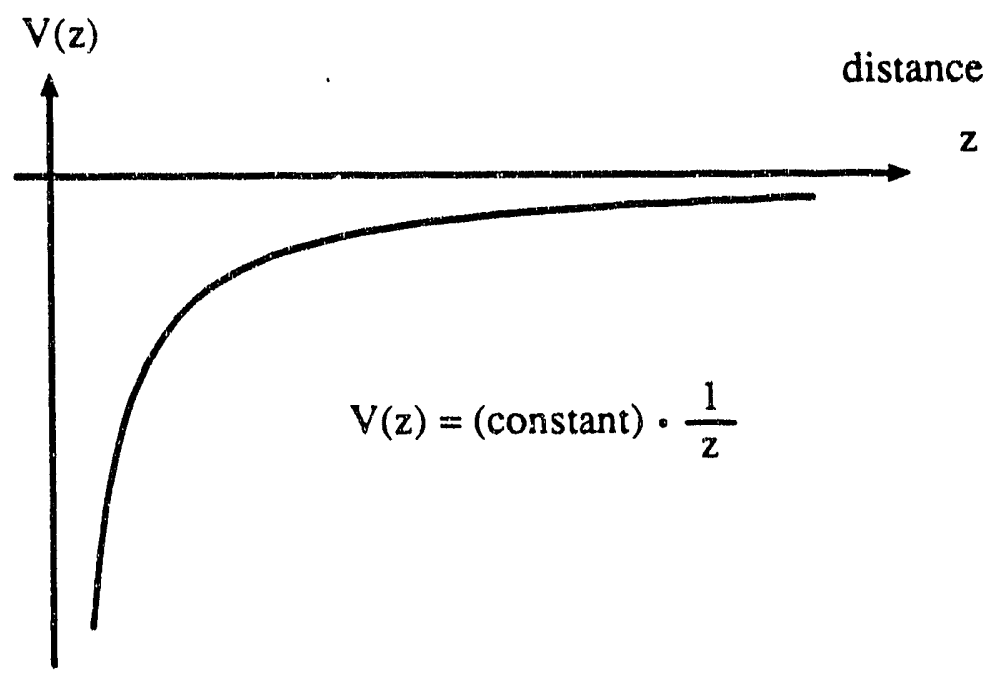

medium

vacuum

Figure 1 
less than the real charge by a factor equal to?

$$
\beta=\left(\frac{e-1}{e+1}\right)
$$

Here, $\epsilon$ is the ratio of the static dielectric constant of the medium to that of vacuum. When its value is equal to $1, \beta$ and the magnitude of the image charge both go to zero. In the limit of $\epsilon$ approaching infinity, which is the case for a metal, $\beta$ approaches 1 .

Referring to the diagram of Figure 1 , the potential energy due to the placement of the point charge $(-q)$ a distance $z$ from the surface has the same form as the potential energy existing between two opposite point charges a distance $2 z$ apart:

$$
V(z)=-\frac{1}{2}\left(\frac{q^{2}}{2 z}\right)\left(\frac{e-1}{\epsilon+1}\right)\left(\frac{1}{4 \pi \epsilon_{0}}\right)
$$

The leading factor of $1 / 2$ is an important but slightly subtle aspect of the problem. While the image force acting upon the real charge $(-q)$ is identical to the force that would exist if the image charge $(+\beta q)$ were a real charge placed $2 z$ away, no such force can be said to be experienced by the image charge, since it has no physical existence. Energy is not stored in the surface charge distibution. Thus, only half as much electrostatic potential energy is contained in the image potential system as would be contained in the corresponding 
arrangement of two real point charges.

An alternate means of understanding why the image potential is exactly half as strong as might be expected requires sonsideration of the energy density stored in the electrostatic image field. For the image potential problem, the distribution of energy density in the half space outside the material is identical to that formed in the equivalent volume between two corresponding point charges having a real physical existence. Integration of the energy density over all space gives the total electrostatic potential energy of the latter system. In the image potential problem, the field terminates at the surface, and there is no energy density stored in the half plane behind the surface. This consideration leads one to conclude that image potential system has half the energy of that in the corresponding system of real charges.

In a special case of particular interest to the present work, the point charge is an electron (whose charge is -e), and the material is a good conductor. Then,

$$
V(z)=-\frac{1}{4 \pi \varepsilon_{0}} \frac{e^{2}}{4 z}
$$

As a demonstration that this image potential function operates on the scale of distance and energy of concern to chemists, one can calculate that an electron an Angstrom from the metal 
surface feels an attraction of 3.6 electron volts.

\subsubsection{Hydrogenic Model}

The theory of bound, quantum mechanical image potential states localized at surfaces has undergone a long evolution as the models used have become more comprehensive and also more complicated. It is worth noting that angular motion does not appear in either the physics or the various formalisms of image potential states. The well-known analogy between image potential states and Rydberg atomic states may lead one to believe that quantized angular motion or at least torsional vibration of the electron may occur due to the attractive image charge residing in the surface. The image charge is in no sense due to a positive hole with an effective mass, but is merely a localization of charge density. It responds instantaneously to lateral motion of the electron occupying the image potential state. No centripetal forces are available in the system to support angular motion.

The hydrogenic model ${ }^{2}$ is the simplest theory of image potential states, and is based on analytic solution of Schrödinger's equation for a one-dimensional analog of the simple hydrogen atom problem. In its simplest form, it. describes a suxface with no layers of adsorbed material. It does not account for or describe penetration of the wavefunction into the surface of the substrate. Thus, it is equally applicable to a variety of systems, but, for the same 
reason, it cannot produce the empirically observed dependence of binding energy upon the crystal nature of the substrate, crystallographic orientation of the surface or momentum of the image potential state in the plane of the surface.

The potential surface used in the hydrogenic model is shown in Figure 2. The substrate is taken to fill the halfspace along the $(-z)$ axis, and the one-dimensional coulomblike image potential is imposed upon the electron outside the surface. The surface provides an infinite barrier to the electron. For the metals considered here, the static dielectric constant is taken as infinite. The wave function obtained is assumed to be separable into $x, y$ and $z$ parts, and the first two are immediately taken to be plane wave solutions. Any periodic potential provided by the surface atoms that may lead to bandgap formation is ignored. No quantum defect ${ }^{3}$ is present in the hydrogenic model.

Schrödinger's equation for the wave function describing motion perpendicular to the surface is, for $z>0$,

$$
-\frac{\hbar^{2}}{2 m_{\theta}} \frac{d^{2} \psi}{d z^{2}}-\frac{z e^{2}}{z} \psi=E \psi
$$

Here, $m_{e}$ is the mass of an electron in free space, $e$ is the magnitude of the electron charge, and, since this problem is that of a one-dimensional hydrogen atom, $z$ plays the role of an effective nuclear charge and has value $1 / 4$ for conductors, which are of interest in the present work. 
Caption for Figure 2:

Energy levels for a single electron outide an ideal conductor whose surface is sharply demarcated at $z=0$. In this hydrogenic approximation, image potential states can be thought of loosely as Rydberg states of the surface. 


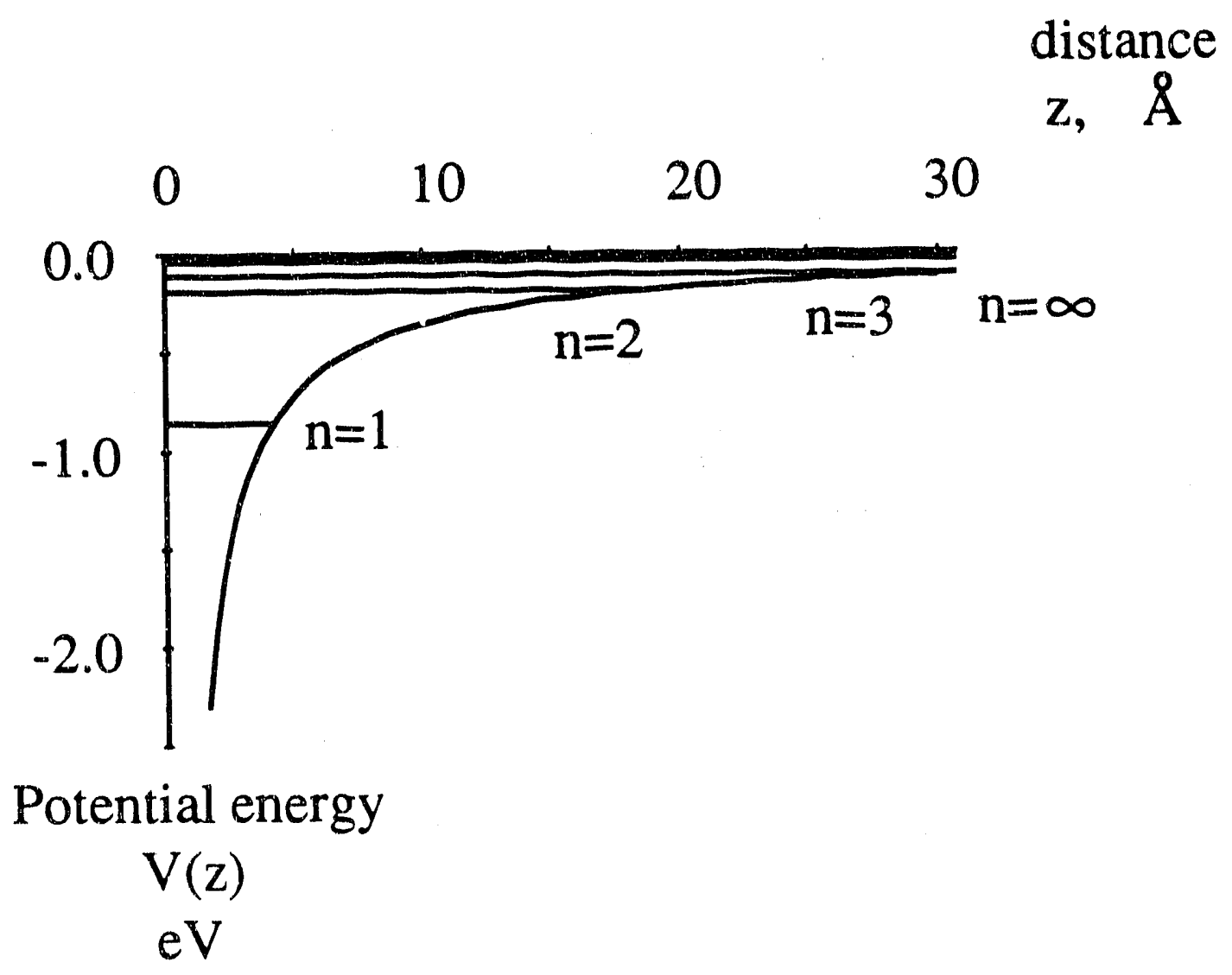

Figure 2 
This equation has the same form as Schrödinger's equation for $P(r)$, which appears in a common solution of the threedimensional hydrogen atom problem. ${ }^{4}$ In this treatment, $P(r)=r \cdot R(r)$, where $r$ is the independent radial variable, and $R(r)$ is the well-known radial part of the hydrogen atom wave function. The variable $z$ can be substituted for $r$, and $R$ is called $\phi / z$. The function $\phi / z$ is then multiplied by $z$ to get the correct expressions for the image potential eigenfunctions. The first three are as follows:

$$
\begin{aligned}
& \Psi_{1}(z)=A_{1} z e^{-\frac{\alpha z}{2}} \\
& \Psi_{2}(z)=A_{2} z e^{-\frac{\alpha z}{2}}(2-\alpha z) \\
& \Psi_{3}(z)=A_{3} z e^{-\frac{\alpha z}{2}}\left(3-\alpha z+\frac{\alpha^{2} z^{2}}{2}\right)
\end{aligned}
$$

where:

$$
\begin{gathered}
A_{n}=\frac{\alpha^{3 / 2}}{n \sqrt{2}} \\
\alpha=\frac{2 Z}{n a_{0}}
\end{gathered}
$$


The quantum number is $n$, and assumes the values $1,2,3 \ldots$ The Bohr length, $a_{0}$, is 0.529 A. The eigenvalues are exactly $1 / 16$ the corresponding values in the hydrogen atom problem, owing to the factor of $\mathrm{z}^{2}$ :

$$
\begin{aligned}
E_{n} & =-\frac{z^{2} m e^{4}}{2 n^{2} n^{2}} \\
& =\frac{-0.85}{n^{2}} \text { electron volts }
\end{aligned}
$$

Using the eigenfunctions obtained, the expectation value of the image potential state electron's distance from the surface for various values of $\mathrm{n}$ is easily calculated. It is simply $6 \mathrm{a}_{0} \mathrm{n}^{2}$. Plots of the probability densities for the electron in the various states are shown in Figure 3 . Expectation values of the electron's distance from the surface as well as the eigenvalues measured relative to the vacuum energy are listed in Table $I$. 
Caption for Figure 3 :

plots of probability densities for the first four image potential states, calculated in the hydrogenic approximation. Arrows denote expectation values of the electron's position, as listed in Table I. 


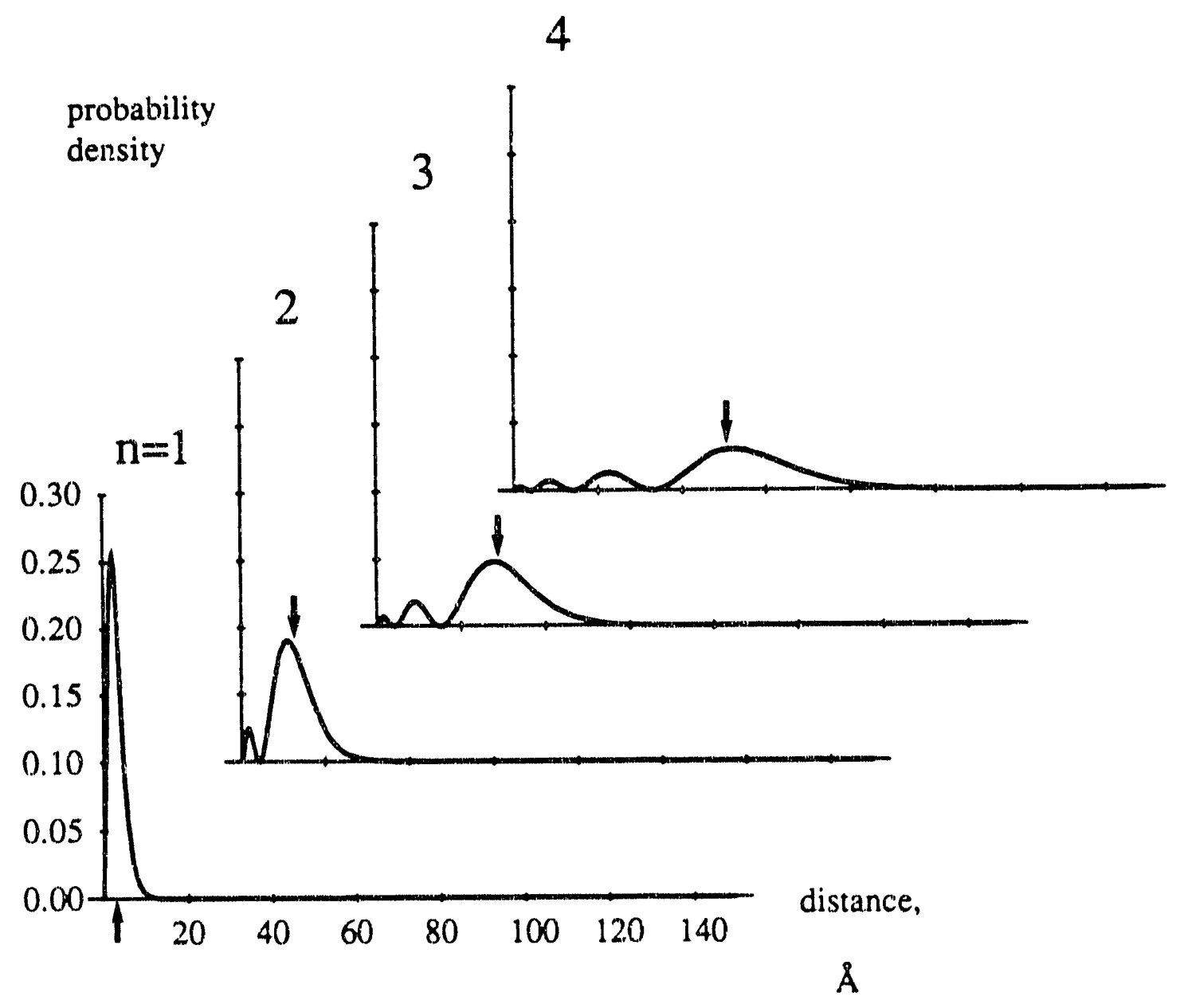

Pigure 3 
Table I Energy levels and expectation values of position in hydxogenic model

\begin{tabular}{|l|l|l|}
\hline $\begin{array}{l}n, \text { quantum } \\
\text { number }\end{array}$ & $\langle z\rangle, \AA$ & $\mathrm{E}_{\mathrm{n}, \mathrm{eV}}$ \\
\hline 1 & 3.18 & -0.850 \\
\hline 2 & 12.70 & -0.210 \\
\hline 3 & 28.58 & -0.094 \\
\hline 4 & 50.80 & -0.053 \\
\hline
\end{tabular}

1.1.3 Multiple Reflection Theory.

\subsubsection{Introduction}

Multiple reflection theory provides the most widely used theoretical description of the behavior of image potential states on metal surfaces. By incorporating penetration of the wavefunction into the surface, it models the effect of the bulk crystal and bulk electronic band structure upon the image potential state. It is useful for obtaining eigenvalues, but lends itself less well to determining the form of the wave function. A further limitation is that it only provides an accurate description of image potential states lying in projected bulk band gaps ${ }^{5}$. Empirically, image potential states can be observed coincident with the continuum of projected bulk bands as well. While multiple reflection theory as it was originally derived applies to surfaces without adsorbed layers, attempts have been made to adapt it 
to treat adsorbate-covered surfaces as well.

\subsubsection{Literature}

Multiple reflection theory for image potential states evolved from the formal treatment of image potential surface resonances, which can be observed in low-energy elecron diffraction experiments. ${ }^{6,7,8,9}$ The use of multiple reflection theory to treat the stationary image potential state was first demonstrated by Echenique and Pendry. ${ }^{10}$ Motivated by the intervening discovery of image potential states by inverse photoemission, 11,12,13, 14,15,16, 17, 18,19 Smith used it several years later to obtain their binding energies on various crystal faces of copper. ${ }^{20}$ Smith's paper also gives early ideas concerning the influence of bulk bands upon the dispersion of image potential states and predicts the existence of energy gaps in the image potential state bands at the surface Brillouin zone ${ }^{21}$ boundary. Subsequently, Smith performed a similar treatment on various faces of nickel ${ }^{22}$ and predicted the existence of a gap in the image potential state band at the boundary of the surface Brillouin zone on $\mathrm{Ni}(110)$. A later paper ${ }^{23}$ focussed specifically on image potential states supported by projected bulk band gaps centered on the zone boundary. Papadia and coworkers ${ }^{24}$ incorporated the effects of surface corrugation into a multiple reflection treatment of image potential states on Al(111). Reviews of the applications of multiple reflection 
theory have been written by $\operatorname{smith}^{25}$ and also by Echenique and Pendry. ${ }^{26}$

Calculations based on multiple reflection theory also appear frequently in the experimental literature concerning image potential states. of particular note is the inverse photoemission work of Hulbert, Johnson and coworkers. They have experimentally studied and applied multiple reflection theory to image potential states on $\mathrm{Ag}(111), 27$ the (111) and (100) faces of copper, 28,29 and $\mathrm{Pd}(111) .{ }^{19}$ Bartynski and coworkers have followed a similar course in studying $\mathrm{Cu}(110) .30$ The (100), (110) and (111) faces of $\mathrm{Ni}, \mathrm{Cu}$ and $\mathrm{Ag}^{31}$ and $\mathrm{Ni}(110)$ with adsorbed $\mathrm{Na}^{32}$ have been examined using inverse photoemission and multiple reflection theory by Dose and coworkers. Lindgren and walldén have used a modified form of multiple reflection theory in explaining data on alkalimetal-induced states on $\mathrm{Cu}(111) .33,34$

Use of multiple reflection theory in explaining twophoton photoemission data on image potential states at metal surfaces is also widespread. Steinmann and coworkers have treated $\mathrm{Cu}(100), 35,50 \mathrm{Ag}(100), 35 \mathrm{Ag}(111), 50$ and $\mathrm{Ni}(111) .35,36$ They have also treated $\mathrm{Cu}(111)$ with adsorbed $\mathrm{Na} \cdot{ }^{37}$ Kubiak and coworkers have combined multiple reflection theory calculations with two-photon photoemission experiments on $\mathrm{Pd}(1.11), 38 \mathrm{Cu}(111)^{39}$ and $\mathrm{Ni}(1.11) \cdot{ }^{40}$ Arguments based on multiple reflection theory are also used in interpretation of data obtained in the present study. 


\subsubsection{Derivation}

In multiple reflection theory (also referred to as phase analysis theory), the image potential state is conceptualized as a plane wave in three dimensions undergoing free propagation in a slab of space between two planar, parallel, perfectly reflecting walls. The reflection process is represented schematically in Figure 4 . One reflecting wall is due to the image potential barrier which prevents the electron from escaping into the vacuum. The other reflecting wall is a barrier to penetration at the surface of the material. In the present case, the material is taken to be a metal crystal. It should be noted that the second wall can only exist when the energy of the electron in the image potential state falls within a projected gap of the bulk electronic band structure. 5 If this condition is not fulfilled, an image potential state may in fact be empirically observable, but multiple reflection theory cannot describe it properly.

since multiple reflection theory treats coupling of image potential states to the bulk lattice, it is necessary to define certain terms of solid state physics before summarizing the derivation of multiple reflection theory. The wave vector $k$ is used as a symbol for the crystal momentum of an electron. This momentum differs from classical momentum by being rigorously conserved only to within an integer multiple of $\mathrm{g}$, the fundamental wave vector of the lattice. For a periodic array of lattice points in one dimension, $g$ is equal to $2 \pi / a$, 
Caption for Figure 4:

schematic drawing depicting the repeated scattering of amplitude flux which is able to generate stationary eigenfunctions in the multiple reflection theory of image potential states. In this drawing, electronic potential energy is plotted along the vertical axis, while position is plotted along the horizontal axis. $\phi_{c}$ is the phase shift suffered by an electron due to the effect of the crystal lattice. $\phi_{B}$ is the phase shift inflicted upon the electron by reflection from the image potential barrier. While the crystal and the barrier are here separated by a free propagation region for the sake of clarity, the width of this region is usually taken as zero. 

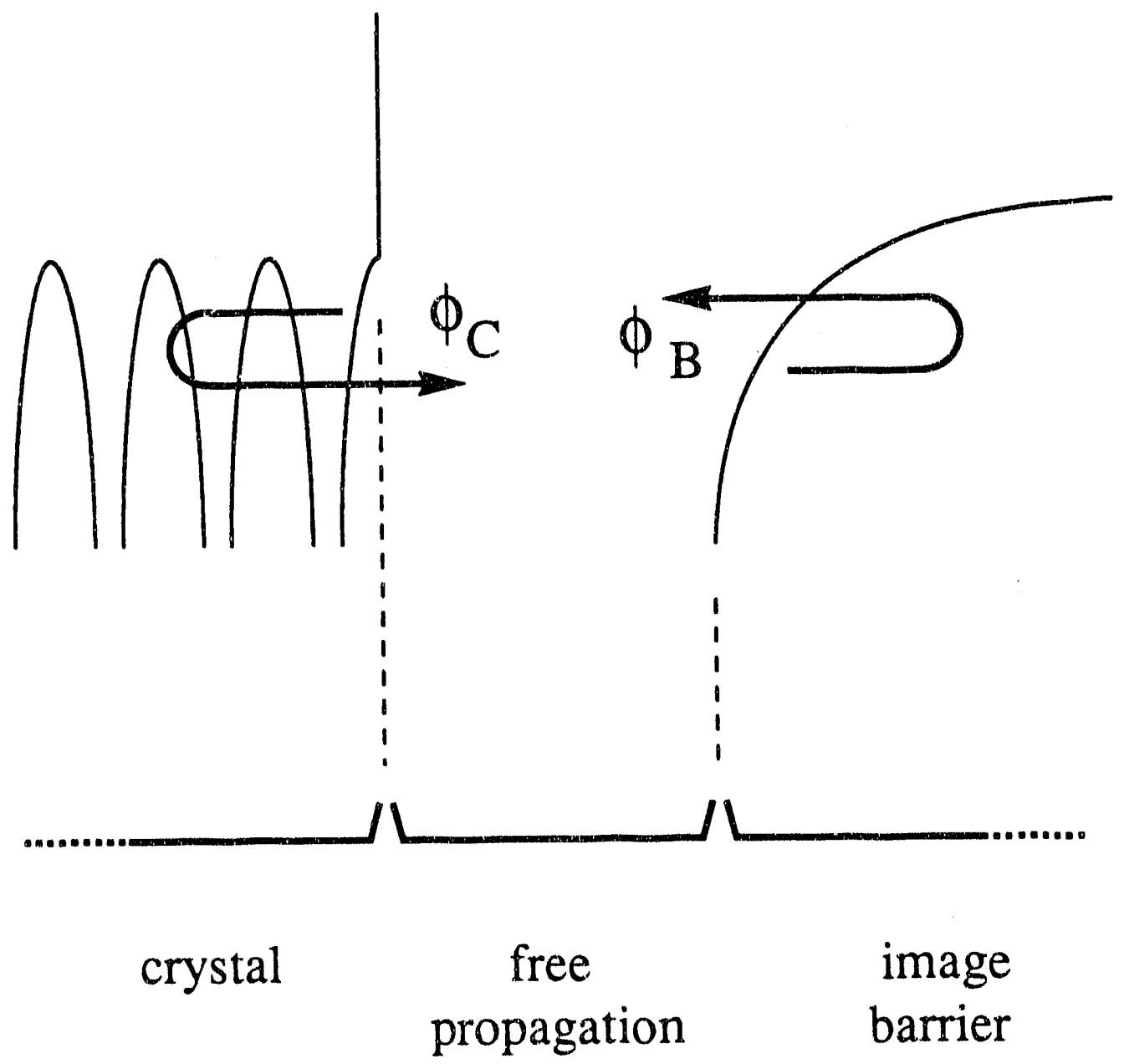

Pigure 4 
where a is the lattice constant. In a solid, $k$ and $g$ are three-dimensional vectors existing in a reciprocal space referred to as $k$-space or momentum space. Also existing in this space is the Brillouin zone, which is a geometrical object whose shape is peculiar to a given type of lattice. The Brillouin zone for the face-centered cubic (FCC) lattice, for example, is shown in Figure 8 on page 66. Electronic eigenvalues form a continuous spectrum over ranges of energy called bands. Gaps in the band structure are created at the boundaries of the Brillouin zone (where $k= \pm g / 2$ ) due to Bragg reflection of the electronic probability waves. points of high symmetry in the Brillouin zone are labelled by upper case letters and correspond to certain directions of low Miller index in the direct lattice. The origin of $\mathrm{k}$-space or, equivalently, the center of the Brillouin zone is labelled $\Gamma$.

Two-dimensional Brillouin zones may be inhabited by electronic states which are localized to a plane surface of a three-dimensional lattice. For such states, the twodimensional wave vector is referred to as momentum in the plane of the surface. It is often given the symbol $k_{1}$. points of high symmetry in the surface Brillouin zone are labelled by upper case letters with a bar overhead. The bulk band structure may be projected into the surface Brillouin zone by integrating over the normal component of the bulk wave vector. A commonly encountered diagram called the projected bulk band structure is generated by plotting electronic energy 
against momentum along a particular line connecting symmetry points in the surface Brillouin zone. Such a projection for Ag(111) is shown in Figure 10 on page 75 . Cuts through the surface state bands are lines. Shaded regions correspond to a continuum of bulk energy and momentum states, averaged over the normal component of the bulk wave vector. Two excellent and general tutorials on this topic are the works of zangwill 5 and Plummer and Eberhardt. ${ }^{21}$

To perform a multiple reflection theory calcuation, attention is focussed on a particular gap in the projected bulk band structure appropriate to a certain crystallographic face of the metal. The calculation is greatly simplified if this bandgap occurs at the center of the surface Brillouin zone of the crystal face of interest. In this case, it is only necessary to consider two fourier components of the three-dimensional potential provided by the lattice of positive ion cores to electrons moving in the metal in order to formally describe the effect of the bulk upon the image potential states. Only projected bulk band gaps at the zone center will be considered in this work.

In the multiple reflection formalism, the wave function of the image potential state may be separated into three parts which are each a function of one Cartesian space coordinate. The part which is a function of $x$ and $y$ represents motion in the two directions in the plane of the crystal surface and is taken to be a plane wave. In other words, momentum in these 
directions is conserved, and the dispersion is that of an electron in two-dimensional free space. This is the same assumption made in the hydrogenic model.

For motion in the direction perpendicular to the surface (z), the image potential state is viewed as being topologically equivalent to the simple quantum mechanical problem of an electron on a ring. In order for a bound state to exist, the phase of the wave function must advance through an integral number of cycles is the amplitude flux contained in the plane wave propagates in a complete circuit between the two reflecting walls. It is this feature of multiple reflection theory which diktinguishes it as a generalization of Maue's original theory of surface electronic states, 4 upon which it is largely based.

Formally, the condition for existence of a bound state is given by the relation: ${ }^{10,20}$

$$
2 \pi n=\phi_{c}+\phi_{b}+2 \pi k I
$$

where $n(=0,1,2 \ldots)$ is the quantum number of the image potential state, $\phi_{c}$ is the phase accumulated in reflection from the crystal boundary, and $\phi_{b}$ is the phase accumulated in reflection from the image potential barrier. The last term on the right is phase accumulated in the process of inward- and outward-directed free propagation through the region of flat potential located between the two barriers. 20,26 The width of this region is specified by the variable 1 . The quantity $k$ is 
an effective free-particle momentum corresponding to the energy $E$ of the image potential state relative to the energy origin of the bulk band structure. It is defined below, with $m_{e}$ being the mass of an electron in free space:

$$
\boldsymbol{K}=\sqrt{\frac{2 m_{e} E}{\hbar^{2}}}
$$

Most often, the reflecting regions of the crystal boundary and image barrier are taken to be immediately adjacent to one another. In this case, 1 is zero, and free-particle propagation makes no contribution to accumulated round trip phase.

The problem of calculating image potential state eigenvalues reduces to that of obtaining expressions for $\phi_{c}$ and $\phi_{b}$ as functions of image potential state energy within the bulk band gap. The eigenvalues are then the energy roots of equation (9) for various values of $n$.

An expression for $\phi_{c}$ is converiently obtained by use of the two-band nearly free electron model applicable to shockley type surface states lying in gaps of the projected bulk band structure. Such states are due to the termination of the bulk lattice, and it is not necessary to invoke image potentials to describe them. The original reference for this theory is the work of Maue. ${ }^{41}$ The later work of Goodwin ${ }^{42}$ extended it slightly further. The contributions of Forstmann $4 j, 44$ 
provide a more modern perspective on the field. It is also covered in the text by Ashcroft and Mermin ${ }^{45}$.

Particular attention is focussed here upon the silver Igap at the center of the (111) suxlace Brillouin zone. This gap occurs between the first and second free-electron-like bands of the bulk metal. The origin of the z-axis is located in the outermost layer of positive ion cores, and the crystal extends toward the negative $z$ direction (to the left). This situation is diagrammed in Figure 5. The surface is taken to be located half an interlayer spacing (a) to the right of the origin. This convention is used by Goodwin, and is the opposite of that used by Maue. The physics that ultimately results is, of course, independent of the choice of either convention.

Schrödinger's equation for bulk electrons moving along the $z$ direction is

$$
-\frac{\hbar^{2}}{2 m_{\theta}} \frac{d^{2}}{d z^{2}} \Psi+V \psi-E \psi=0
$$

where the potential is a Fourier series

$$
V(z)=\sum_{n=-\infty}^{n=\infty} V_{n} e^{i n g z}
$$

and $g=\pi / a$.

Following smith's convention, ${ }^{20} V_{0}$, the coefficient of 
Caption for Figure 5:

a) Lattice in direct space, truncated at the surface. The origin is taken on a surface atom. The heavy curve is the the potential energy of the electron.

b) Iowest two free-electron-like bands, showing midgap energy $\left(E_{g}\right)$ and gap width $\left(2 \mathrm{~V}_{g}\right)$. The origin of energy for nearly free electrons in the bulk is the bottom of the first band, at $k=0$. 
a)

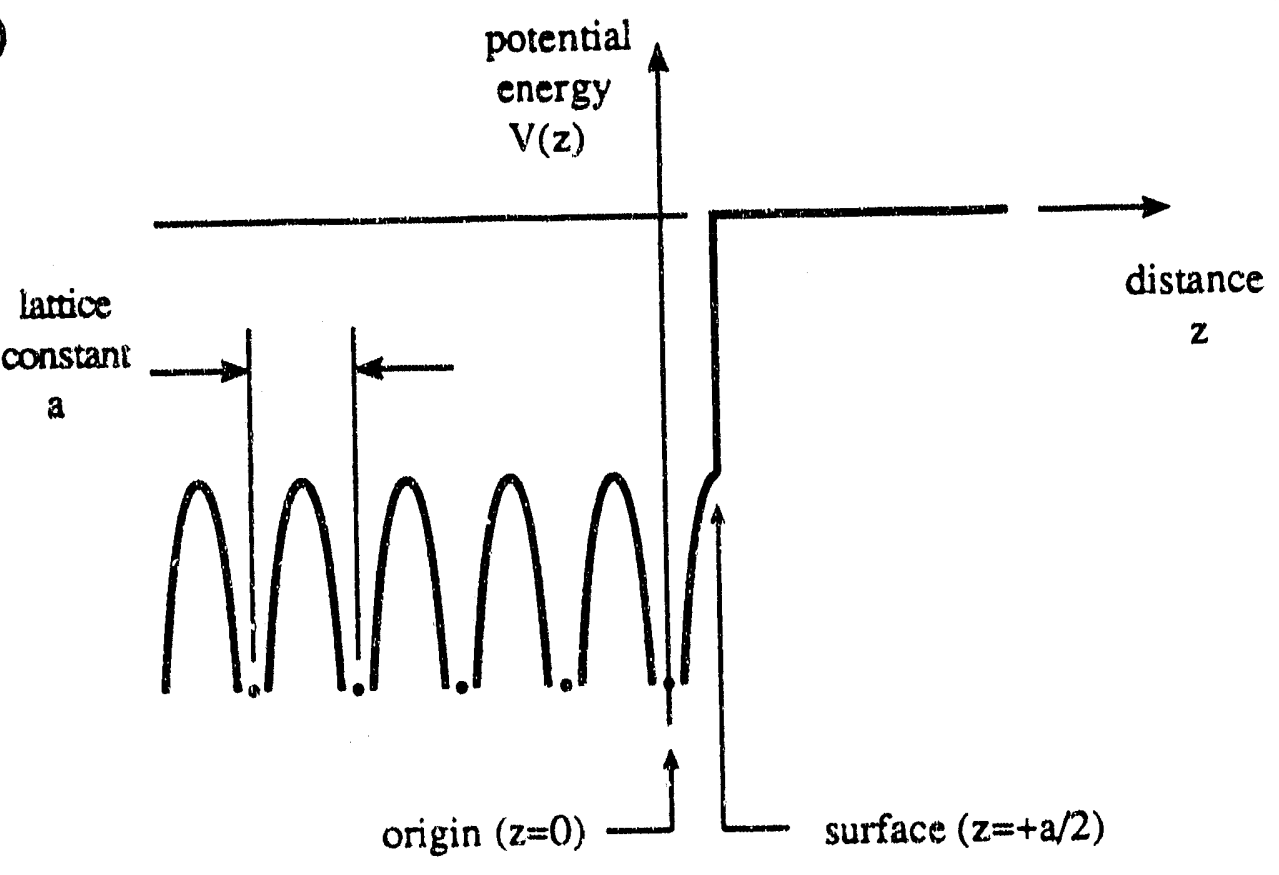

b)

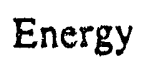

Figure 5

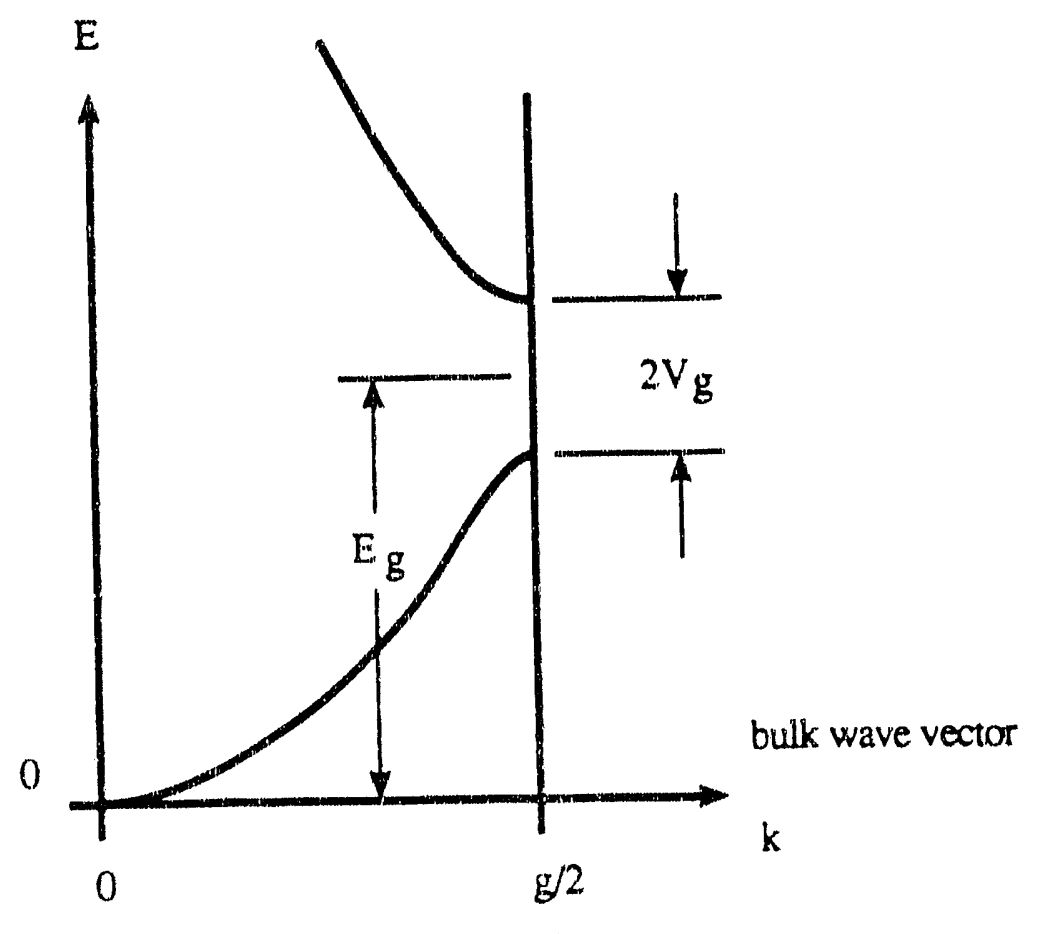


the first term in the series, is conveniently taken as'zero. Thus the energy origin for the whole problem is the bottom of the lowest bulk free-electron-like band, as indicated in Figure 5.

The two-band approximation is used when the wave function $\checkmark$ is taken as consisting of two terms corresponding to pure momentum states near the boundary of the first Brillouin zone at $k=g / 2$ (the symmetry point labelled as $L$ ).

$$
\psi=\alpha e^{i k z}+\beta e^{i(k-g) z}
$$

When $k=g / 2$, is real and is a standing wave whose phase is determined by coefficients $\alpha$ and $\beta$. This is the wave function appropriate to bulk states exactly at the energy of the upper and lower projected bulk band edges. Inside the gap, $k$ is complex. Its real part is fixed at value $\mathrm{g} / 2$ (called $\mathrm{p}$ ), and its imaginary part (called q) ranges in a smooth fashion from zero to some extreme value and back to zero as the gap is traversed. This graphical view of the complex wave vector has been advanced in the literature by Heine. ${ }^{46}$ A schematic diagram is shown in Figure 6 .

In the gap, the wave function is oscillatory, decays exponentially into the bulk, and has minimal penetration near the midgap energy where $q$ reaches its maximum. Formally,

$$
\begin{gathered}
k=p+i q \\
k-g=-p+i q
\end{gathered}
$$


Caption for Figure 6:

Energy of nearly free electrons in the bulk as a function of complex wave vector. Electronic motion in only one spatial dimension, that parallel to $\Gamma-L$ in the FCC Brillouin zone, is treated in this diagram. As the band gap is traversed from top to bottom, the total energy increases smoothly as the imaginary part of $k$ ranges from 0 to its extremal value ( $\left.q_{\text {extr }}\right)$, and back to zero. While wave vector may be complex, energy remains real. 


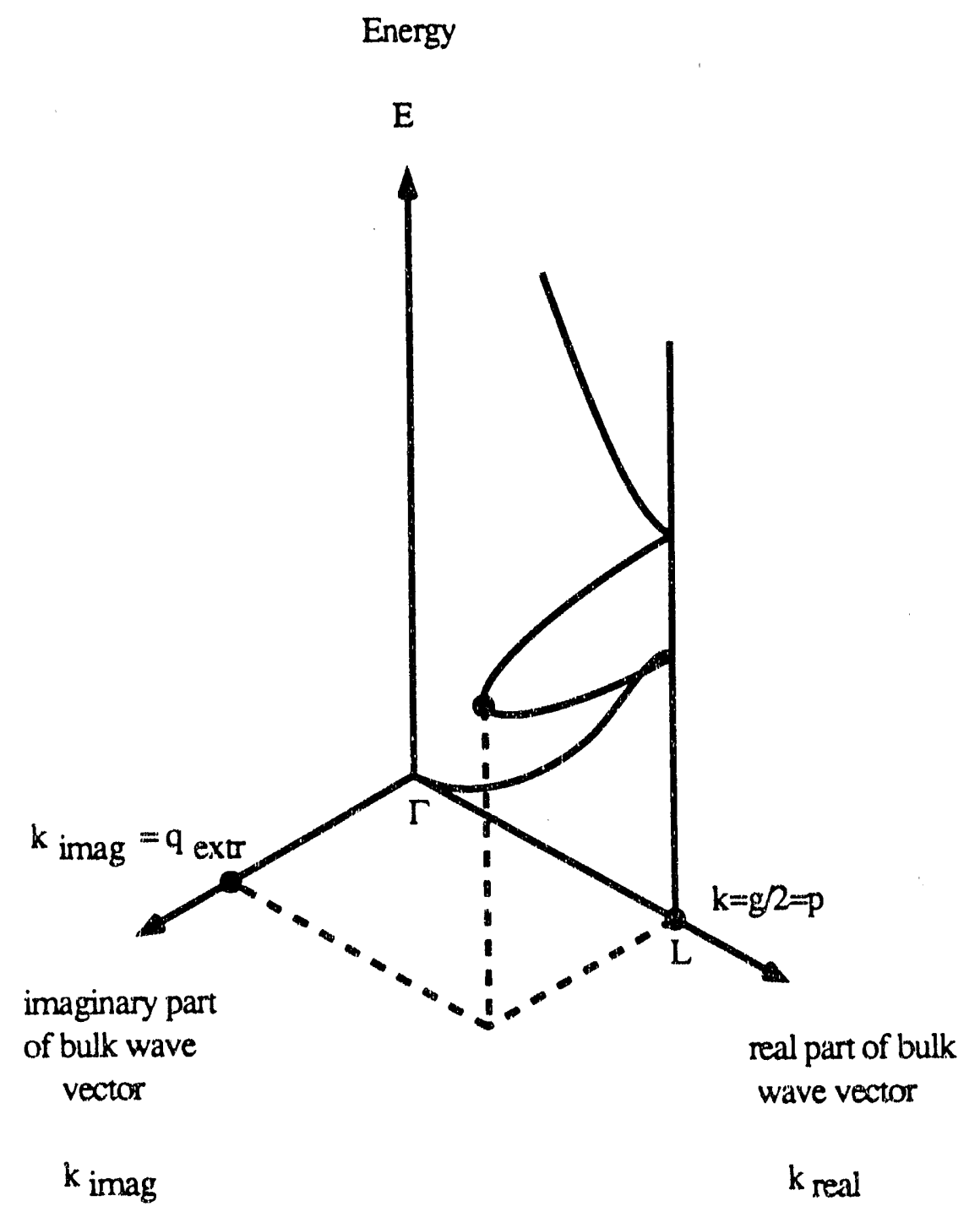

Iguxa 6 


$$
\begin{aligned}
\Psi & =\alpha e^{i(p+i q) z}+\beta e^{1(-p+i q) z} \\
& =e^{-\alpha z}\left(\alpha e^{i p z}+\beta e^{-i p z}\right)
\end{aligned}
$$

$$
\frac{d^{2}}{d z^{2}} \Psi=(q-i p)^{2} \alpha e^{i(p+i q) z}+(q+i p)^{2} \beta e^{i(-p+i q) z}
$$

Because only bulk states near the first gap in the bulk freeelectron bands are considered in equation (13), only the terms $V_{1}$ and $V_{-1}$ in the periodic potential (equation (12)) need to be used in solving schrödinger's equation.

Equation (11) becomes a pair of linear equations, which, after substitution of equation (15) and equation (16) and elimination, yield a pair of linear equations in $\alpha$ and $\beta$. For the moment, units are taken to be such that $c$ (below) is defined as one:

$$
c=\sqrt{\frac{\hbar^{2}}{2 m_{\theta}}}
$$

The linear equations are:

$$
\begin{aligned}
& {\left[(q-i p)^{2}+E-V_{0}\right] \alpha+\left[-V_{g}\right] \beta=0} \\
& {\left[-V_{g}\right] \alpha+\left[(q+i p)^{2}+E-V_{0}\right] \beta=0}
\end{aligned}
$$


Here, $v_{0}=V_{1}=V_{-1}$ is the real coefficient of the fundamental component of the periodic potential. The requirement that $\alpha$ and $\beta$ be not both equal to zero results in the secular equation below:

$$
\left|\begin{array}{lr}
q^{2}-2 i p q-p^{2}+E & -V_{g} \\
-V_{g} & q^{2}+2 i p q-p^{2}+E
\end{array}\right|=0
$$

Evaluating the determinant gives a quadratic in the eigenvalue E:

$$
E^{2}+E\left(2 q^{2}-2 p^{2}\right)+\left(q^{4}+p^{4}+2 p^{2} q^{2}-V_{g}^{2}\right)=0
$$

Using the quadratic formula to solve for $\mathrm{E}$,

$$
E=p^{2}-q^{2}+V_{0} \pm \sqrt{V_{g}^{2}-4 p^{2} q^{2}}
$$

Insertion of either root of equation (22) into equation and equation (19) gives the results

$$
\begin{aligned}
& (-2 i p q+\Delta) \alpha+\left(-V_{g}\right) \beta=0 \\
& \left(-V_{g}\right) \alpha+(2 i p q+\Delta) \beta=0
\end{aligned}
$$

Here, $\Delta$ is defined as 


$$
\Delta=\sqrt{V_{g}^{2}-4 p^{2} q^{2}}
$$

The mixing coefficients $\alpha$ and $\beta$ above are taken to be complex conjugates and of magnitude equal to one:

$$
\alpha=e^{i \delta}=\beta^{*}
$$

Thus, the wave function (13) can be written as

$$
\psi=e^{-q x}(\cos [p z+\delta])
$$

An expression for $\delta$ is obtained from solution of equation (12) and equation (24) through the use of equation (26):

$$
\begin{gathered}
\sin (28)=\frac{1}{2 i}\left(\frac{4 i p q}{V_{g}}\right)=\frac{2 p q}{V_{g}} \\
\delta=\frac{1}{2} \arcsin \left(\frac{2 c^{2} p q}{V_{g}}\right)
\end{gathered}
$$

The constant $c^{2}$ has been explicitly reinserted in equation (29).

Because $v_{g}$ is positive for electrons propagating along the $\Gamma-I$ direction in silver, the wave function at the upper band edge of the L-gap are s-like, while those at the lower edge are $\mathrm{p}-1 i k \mathrm{e}^{47,43}$. In other words, the standing waves at the top of the gap have nodes between ion cores, but those at 
the bottom (at lower energy) have nodes centered on ion cores. This is the phenomenon referred to as shockley inversion or s$p$ inversion. It arises physically because the periodic potential along the (111) direction has upward wiggles at the sides of the positive ion cores. These wiggles in the potential are directional and are due to the influence of occupied bands derived from atomic d-orbitals. The wiggles are of sufficient magnitude along the $\Gamma-L$ direction to make the sign of $v_{g}$ change from the negative value which is generally expected to the positive value which is actually observed.

For such s-p inverted gaps, a correct branch of the arcsin function in equation (29) is chosen by requiring that $\delta$ assume a value of 0 at the top of the gap, $-\pi / 4$ near the middle of the gap and $-\pi / 2$ at the bottom of the gap. Thus the phase of the wave function (equation (27)) advances smoothly from the p-like function at the bottom of the gap to the slike function at the top.

A useable expression for $q$ is obtained by rearrangement of equation (22) and reinsertion of the constant c:

$$
q=-\frac{1}{c}\left(\left[4 E E_{g}+V_{g}^{2}\right]^{\frac{1}{2}}-E-E_{g}\right)^{\frac{1}{2}}
$$

Here, $E_{g}$ is the midgap energy at the $L$ point. Referring to the effective mass of bulk electrons with wave vector along $\mathrm{r}$ - 
L as $\mathrm{m}^{*}, \mathrm{E}_{\mathrm{g}}$ is related to $\mathrm{p}$ by the equation:

$$
E_{g}=c^{2} p^{2}=\left(\frac{\hbar^{2}}{2 m^{*}}\right) p^{2}
$$

The simplifying assumption is thus made that the bulk bands of interest are parabolic near the center of the Brillouin zone. The effective mass is the parameter controlling the band's curvature.

As can be verified, expression (30) for $q$ is zero at both band edges, is negative in between, and has a extremum near the midale of the gap which is given by the expression:

$$
q_{\min }=\frac{-V_{g}}{c^{2} g}
$$

Note that $q$ is not exactly symmetrical about the midgap energy, nor does it reach its extremum there. The minimum penetration of the oscillatory wave function (equation (27)) into the bulk is determined by $q$ and is on the order of several interlayer spacings.

Finally, an expression for $\phi_{c}$ is obtained by matching logarithmic derivatives of the wave function (equation (27)) inside the crystal with the plane wave whose effective wave vector is $k$ (given by equation (10)), which is taken to exist outside the crystal. This external wave function is given below: 


$$
\Psi_{\text {outer }}=e^{-i k z}+I_{c} e^{i \phi_{c}} e^{i k z}
$$

The first term represents flux incident on the crystal, and the second term represents flux travelling to the right after having sufferred reflection and a phase shift of $\phi_{c}$. The reflection coefficient $\left(r_{c}\right)$ is taken to be one since, under the assumptions in this treatment, no net flux can be lost into the crystal.

The first expression below results from matching logarithmic derivatives of the two wave functions, and the second is a rearrangement:

$$
\begin{gathered}
\kappa \tan \left(\frac{\phi_{c}}{2}\right)=p \tan (p z+\delta)+q \\
\phi_{c}=2 \arctan \left(p \tan [p z+\delta]+\frac{q}{k}\right)
\end{gathered}
$$

It is important that the correct branch of the arctangent function in equation (35) be chosen. For $s-p$ inverted gaps such as the silver L-gap, $\phi_{c}$ is expected to evolve from zero at the lower band edge to $\pi$ at the upper band edge. Outside of the gap, $\phi_{c}$ is not defined in this treatment. In practice, the energy-dependence of $\phi_{c}$ is obtained by inserting the expression for $q(E)$ into that for $\delta$, and then inserting $\delta, q$ and $x$ into the expression for $\phi_{c}$. 
The procedure for obtaining the energy-dependence of $\phi_{B}$ is less complicated. Solutions for schrödinger's equation for the image potential are obtained as whittaker functions.7,10,48,49 These functions exhibit a continuous dependence upon the energy of the image potential state. By requiring these solutions to have zeros at the origin, the set of discrete energy values given below is obtained:

$$
E-E_{\text {vac }}=-\frac{13.6}{16 n^{2}} \text { electron volts } \quad n=1,2,3 \ldots
$$

Here, $E_{v a c}$ is the energy of the vacuum level. For these energies, the phase shift sufferred by a wave reflected from the image barrier is an odd, positive integer multiple of $\pi$. An interpolation formula between these points gives a convenient expression for $\phi_{B}$ :

$$
\phi_{B}=\pi\left(\sqrt{\frac{-13.6}{4\left(E-E_{v a c}\right)}}-1\right)
$$

Less tractable, but purportedly more physically accurate, means of obtaining $\phi_{B}$ are based on the assumption that the image well is truncated or saturated at some particular energy such as the mean inner potential of the bulk crystal. In this case, numerical integration of schrödinger's equation is used to evaluate $\phi_{B}$ as a function of energy. It is interesting to 
note that the use of Whittaker functions is an alternate and completely equivalent means of solving cole's hydrogenic model. They are simply more esoteric than the modified hydrogenic radial functions which are familiar from elementary treatments of the hydrogen atom.

Eigenvalues are determined as the energies where equation (9) is satisfied. In s-p inverted gaps, a solution exists near the lower band edge where $\phi_{B}=\phi_{C}$. This is the well-known occupied surface state which emerges from the original theories of Maue and Goodwin. It is often referxed to in the literature of multiple reflection theory as the $n=0$ state. For $n=1,2,3 \ldots$ image potential state eigenvalues on a metal surface are generally well fit by an expression of the form

$$
E_{n}-E_{\text {vac }}=-\left(\frac{13.6}{16(n+a)^{2}}\right) \text { electron volts }
$$

Here, a acts as a quantum defect parameter analogous to that appearing in the theory of Rydberg atoms. This quantum defect is determined by $\phi_{C}$ :

$$
a=\frac{1}{2}\left(1-\frac{\phi_{c}}{\pi}\right)
$$

As defined, a is not generally expected to be a constant since $\phi_{c}$ is not. Often, however, the width of the spectrum of image potential state eigenvalues (excluding the $n=0$ state) is much 
less than the width of the projected bulk band gap. In such a case, $\phi_{c}$ is approximately constant and can be equated to its average value in the region of the image potential states. The quantum defect will then assume a value between 0 and $1 / 2$, depending on the location of the image potential state series in the band gap. For Ag(111), image potential states occupy approximately the upper fifth of the gap, where $\phi_{c}$ is nearly equal to $\pi$. Thus, the quantum defect on this surface is practically zero, and the binding energy predicted by the simple hydrogenic model $(0.85 \mathrm{eV}$ for $\mathrm{n}=1)$ is close to that calculated by multiple reflection theory $(0.75 \mathrm{eV}$ for $n=1$, according to Giesen, et $a 1^{50}$ ).

Image potential states are often referred to as barrierinduced states ${ }^{10}$. This terminology is adopted to emphasize that their existence is due primarily to the image barrier, and that the influence of the substrate is of secondary importance. Multiple reflection theory clearly illustrates this point. The series of image potential states converging strongly upon the vacuum energy is due to rapid oscillations in the wave function induced by the asymptotic tail of the image well. Thus, a large amount of the total phase shift which generates the image potential state is induced by the image barrier and, although the crystal lattice plays a role in determining the exact energy of the levels, it is unimportant to the gross structure of the image potential series. The nature of regular surface states, on the other 
hand (such as the occupied L-gap surface state on Ag(111)) is determined overwhelmingly by the bulk crystal, and these are therefore referred to as crystal-induced states.

\subsubsection{Efective Mass}

Multiple reflection theory may be used to evaluate the dependence of total energy of the image potential state upon momentum (or wave vector) in the plane of the surface. This is referred to as the dispersion relation. Its form is determined by the shape of the projected bulk band edges delineating the gap which the image potential state inhabits. one could imagine finding the dispersion relation by recalculating, point by point, the binding energy of a particular member of the image potential series at a set of gradually increasing values of momenta in the surface plane. Then the binding energy would be seen to vary smoothly with momentum as the bulk band edges dispersed. Essentially, the quantum defect (equation (39)) would become a function of momentum, and this effect would produce the observed variation in the image potential state eigenvalue.

Although the eigenvalue's dependence upon momentum is not. expected to be a simple functional form, it is not drastically different from the parabolic dispersion relation of a free electron. The effective-mass approximation is thus realistic near the center of the surface Brillouin zone and most of the way out to the zone boundary. $\operatorname{sinith}^{50}$ in a paper written with 
Giesen and coworkers, has devised a convenient method of estimating the effective mass which avoids the laborious, repetitive calculations of the eigenvalue. It is based on the simple realization that, when the inage potential state dispersion relation crosses one of the band edges into the continuum of bulk bands, the phase shift inflicted at the crystal boundary is either 0 or $\pi$.

Examining the case of the L-gap on Ag(111) (which is s-p inverted), the energy of the $n=1$ image potential state calculated by nultiple reflection theory at the zone center $\left(E_{\text {MRT }}\right)$ is quite close to the energy of the upper band edge at the zone center $\left(\Sigma_{\mathrm{UBE}}\right)$. This situation is shown in Figure 7 . Since in this case it is safe to assume the dispersion of the image potential state will cross the upper band edge, the total energy of the image potential state where it crosses into the bulk continuum $\left(E_{c}\right)$ is taken to be the sum of the binding energy which results from a crystal-induced phase shift of exactly $\pi(-0.85 \mathrm{eV}$ for $\mathrm{n}=1)$ and the energy associated with parallel motion, which will depend upon effective mass and is to be determined forthwith. The binding energy of $-0.85 \mathrm{ev}$ is, of course, simply the value computed in the hydrogenic model.

The location of $E_{c}$ is determined by considering a hypothetical dispersion curve which has a perpendicular binding energy of $0.85 \mathrm{eV}$ throughout the entire surface Brillouin zone, and thus has an effective mass equal to that 


\begin{abstract}
Caption for Figure 7:
Detail of top of projected bulk I-band gap on silver (111), with parameters labelled for calculation of image potential state effective masses in the manner of Giesen, et al. $E_{\mathrm{FE}}$ is the energy of the free-electron parabola at the zone center. $E_{\text {MRT }}$ is the binding energy of the image potential state calculated by multiple reflection theory. $E_{\text {UBE }}$ is the energy of the upper projected bulk band edge at the zone center. $E_{c}$ is the energy at which the three curves intersect.
\end{abstract}




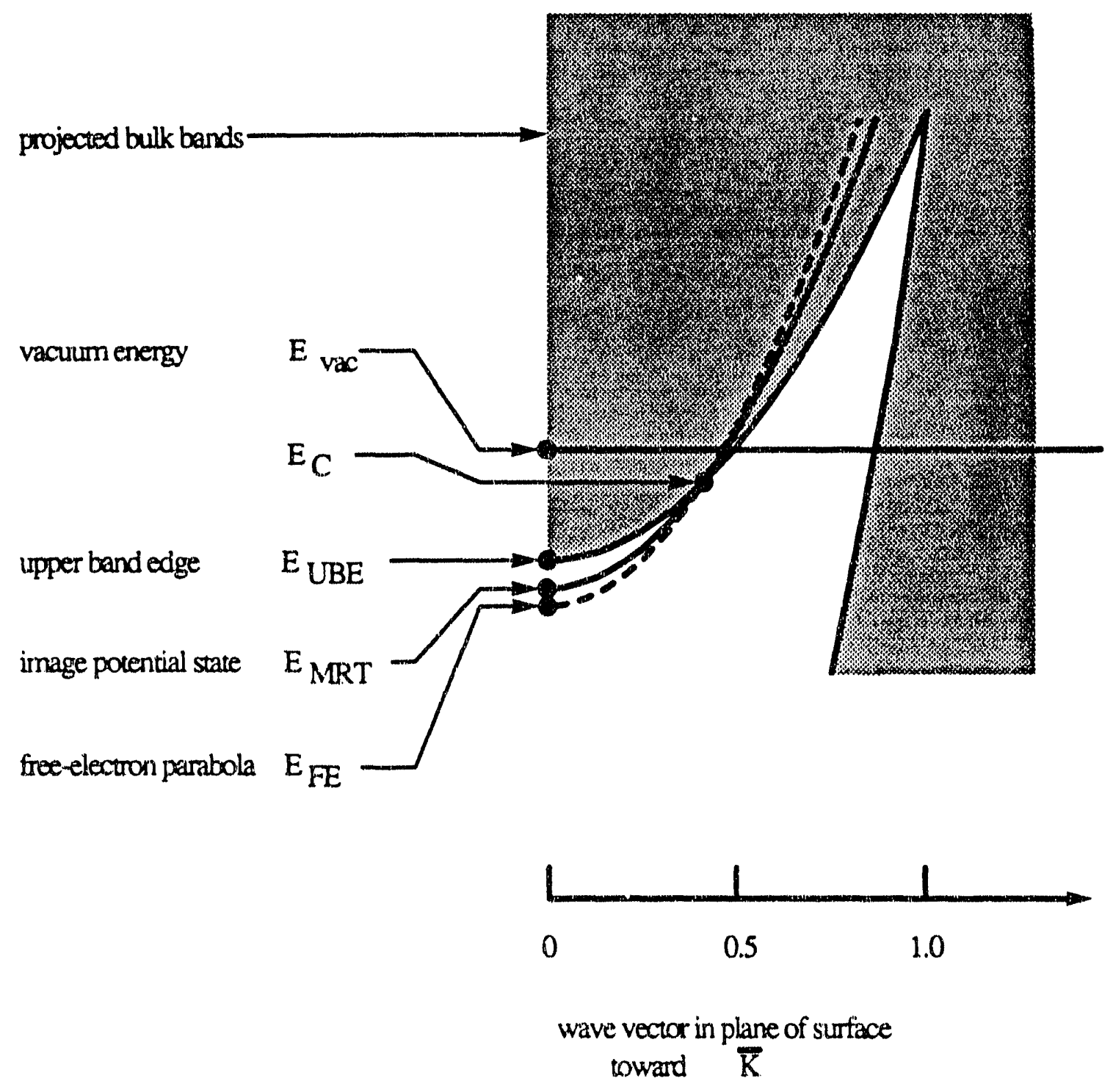

Pigure 7 
of the electron in free space $\left(m_{e}\right)$. This is shown in Figure 7 by the energy $E_{F E}$ and the dotted parabola. The energy at which this curve enters the bulk continuum then is the required value of $\mathrm{E}_{c}$, since the perpendicular binding energies in both dispersion relations must be equal there. The energy of the upper band edge at the zone center $\left(E_{U B E}\right)$ and its effective mass are, of course, necessary to evaluate $E_{c}$. The effective mass ( $\left.\mathrm{m}^{*}\right)$ of the image potential state is then obtained from the relation

$$
\frac{m^{*}}{m_{\theta}}=\frac{E_{C}-E_{P E}}{E_{C}-E_{M R T}}
$$

crudely, the bulk band edges may be said to repel the dispersion curve of the image potential state, so that, when closest to a band edge with a large effective mass, the image potential state acquires a dispersion relation characterized by a large effective mass.

If the image potential state is situated in the gap so that it crosses the edge with zero crystal-induced phase shift (the lower edge, if the gap is $s-p$ inverted). the binding energy of the hypothetical free-electron dispersion curve does not assume the hydrogenic value. It is, instead, the value that results when the quantum defect is equal to $1 / 2$. This is $0.38 \mathrm{eV}$ for the $\mathrm{n}=1$ state. Thus, in general, 0.38 and $0.85 \mathrm{eV}$ are the two possible values for $E_{F E}$ of the $n=1$ state. 
As Smith points out, an image potential state located near the midale of a gap is difficult to treat by this approach. This is because it is necessary to decide which band edge the image potential state's dispersion curve crosses. If this decision is not obvious upon inspection of the projected bulk band structure, the value for the effective mass which results may be completely wrong. Repetitive, point-by-point calculation of image potential state eigenvalues may be necessary to determine the dispersion relation in such a case.

As the boundary of the surface Brillouin zone is approached, Bragg diffraction of the in-plane part of the image potential state's wave function by the periodic array of surface atoms becomes more significant. This effect cannot be accounted for by the present model or by any model where only two fourier components of the potential are considered.

\subsection{4 other Theories}

In addition to the hydrogenic model and multiple reflection theory, several other theoretical approaches to image potential states have been developed. Self-entrgy formalism and the solution of density functional equations are both prominent in the literature. A set of papers by various authors which do not fall into these somewhat restictive categories are also worth mentioning. This work will be considered here as well. 
Theories of image potential states based upon self-energy formalism are due chiefly to two groups. Echenique and coworkers 51,52 initially used such methods to predict a scaling of image potential state lifetimes with the cube of the quantum number $n$. The authors also point out the obvious effect of this trend upon widths of the vaxious members of the image potential series. Subsequently, they also treated the dependence of binding energy and lifetime upon momentum in the plane of the surface. ${ }^{53}$ Two other, more recent articles have also dealt with lifetime calculations. 54,55

zheng and coworkers ${ }^{56}$ have also made use of self-energy formalism. They focussed on applying higher-order perturbation corrections to the image potential itself. Bound quantum states are not treated. In a later article, ${ }^{57}$ similar methods were employed, and important interactions between the image potential and surface plasmons were predicted.

A few theoretical workers have developed a theory of image potential states based on solution of the density functional equations. Hulbert and coworkers ${ }^{58}$ treated the cu(100) surface and reported excellent agreenent of their calculated energies with experimental values obtained by inverse photoemission spectroscopy. The local-density approximation was used in this work. Gies ${ }^{59}$ as well as ossicini and coworkers ${ }^{60}$ have performed similar calculations using the nonlocal-density approximation. Most recently, 
Kiejna ${ }^{61}$ used density functional theory to match the oneelectron effective potential self-consistently with the image potential. The image plane position was calculated for a jellium surface and for several models of real metals.

occasionally, theoretical papers have appeared attempting to treat the effect of the crystal lattice upon the image potential state by much of the same reasoning used in multiple reflection theory. These models are not cast in terms of phase shifts, but rely on wave function matching arguments. The earliest is due to Garcia and solana, 48 and a more recent one is by Weinert and coworkers, ${ }^{62}$ who were chiefly concerned with the location of the image plane. Lenac and coworkers ${ }^{49}$ attempted a similar treatment of Pd(111) and Pd(100) with adsorbed hydrogen.

Th.e recent theoretical contribution of van siclen ${ }^{63}$ is the latest in a lively series of publications concerning the possible effect of surface corrugation upon the binding energy and effective mass of image potential states on metal surfaces. This discussion was initiated by Garcia and coworkers, $^{64}$ who predicted surface corrugation to have a large effect upon the image potential state. Steinmann and coworkers $^{65}$ and Straub and Himpsel $1^{66}$ as well as Hulbert and coworkers $^{58}$ all argued the effects were negligible. Van siclen reached the same conclusion. Compelling evidence has not emerged to support the claims of Garcia, et al.

Two other articles which are not directly applicable to 
the present research nonetheless bear mentioning, if only to demonstrate the range of interesting problems presented by image potential states. Shakeshaft and spruch $^{67}$ calculated cross sections for photoelectron excitation from an initial state of an electron bound to a wall by the image potential. Initial states were taken to be those resulting from the hydrogenic approximation. The same dependence of cross section upon final state kinetic energy was predicted as is observed for the hydrogen atom. Grinberg ${ }^{68}$ examined theoretically the image potential existing in a heterojunction formed in the interface between two dissimilar semiconductors. Important phenomena concerning image potentials are definitely not restricted to those exhibited by metal-vacuum interfaces, which have so far been most thoroughly studied.

\subsubsection{Adsorbate Theories}

The presence of adsorbate atoms or molecules on a surface can have a significant effect upon the image potential state. A variety of theoretical approaches have been suggested to deal with adsorbate systems. One important effect can readily be treated by multiple reflection theory. This is the shift of the work function, which moves the image potential state within the projected gap, leading to changes in its dispersion relation. Such a calculation was used in the present study, but has not been described specifically in the literature.

The hydrogenic model has been adapted to describe certain 
effects induced by dielectric adsorbates upon the image potential state. The simplest effect to treat is the dielectric screening of the image field by the adsorbate layer. For a monolithic slab of dielectric adsorbate whose thickness is practically infinite compared to the spatial extent of the image potential state wavefunctions, the eigenvalues would be reduced by a factor equal to $\epsilon^{-2}$, where $\epsilon$ is the static dielectric constant of the adsorbed material.69 A more realistic but more complicated theory incorporating a layer of finite thickness and the image field induced at the adsorbate-vacuum interface was developed by cole. ${ }^{70}$ Unfortunately, this theory treats the image potential state by assuming that its wave function penetrates only negligibly into the dielectric adsorbate layer. The layer is taken to have a negative electron affinity, producing a repulsive barrier. This assumption may be quite valia for layers of light noble gases on metals, for which the theory was developed, but its applicability to the present research involving heavy noble gases and organic molecules is doubtful. It should also be noted that cole's calculations dealt chiefly with layers whose thickness corresponds to tens of atomic monolayers or more.

Another treatment of image potential states in the presence of adsorbed insulating materials was reported by Trninić-Radja and sunjić, ${ }^{71}$ working in collaboration with Lenac. This was a variational calculation based upon wave 
functions obtained from the hydrogenic approximation. Like Cole's theory, this work also includes the assumption of a strong repulsive barrier at the dielectric-vacuum interface. Also like cole's theory, it is intended to describe systems where the adsorbate thickness is tens of monolayers or more. Neither of these theories is used in interpreting the results of the present experimental work.

\subsection{Experiments on Image Potential states}

\subsubsection{Inverse Photoemission}

An inverse photoemission experiment is performed by firing a monochromatic electron beam at a metal surface. At the surface, a very small fraction of the free electrons in the beam undergo radiative transitions to unoccupied electronic states of the metal. The emitted light is traditionally called bremsstrahlung, and its spectrum gives the differences between the beam energy and the various final states. In the most comnon experimental arrangement, referred to as bremsstrahlung isochromat spectroscopy, ${ }^{18}$ the spectrum is accumulated by scanning the beam energy and detecting photons of fixed energy. This energy is typically $9.7 \mathrm{ev}$, due to the widespread use of iodine-filled Geiger-Müller tubes as detectors. The energy resolution obtained by this means is 200 to $500 \mathrm{meV}$. Since the in-plane component of electron momentum is conserved in the radiative transition, the dispersion of the final state's energy with momentum can be 
determined merely by varying the angle of incidence of the beam relative to the surface normal. An experiment of this type is commonly referred to as "k-resolved inverse photoemission," often abbreviated to "KRIPES." Inverse photoemission, since it is sensitive to empty levels, is well suited to studying image potential states as well as unoccupied negative ion levels of adsorbates on metals. The field has been reviewed by $D_{0 s} 72,73$ and more recently by Smith. ${ }^{25}$ The latter review is from a theoretical viewpoint.

Three measurements of the binding energy and dispersion of the $n=1$ image potential in the L-gap on Ag(111) have been reported. Hulbert and coworkers ${ }^{27}$ obtained a binding energy of $0.7 \mathrm{eV}$ and a dispersion along the $\bar{\Gamma}-\overline{\mathrm{K}}$ azimuth characterized by effective mass $\mathrm{m}^{*}=0.7 \mathrm{~m}_{e}$. Goldmann and coworkers ${ }^{31}$ reported a binding energy of $0.6 \pm 0.2 \mathrm{ev}$ and $\mathrm{m}^{*}=(1.4 \pm 0.3) \mathrm{m}_{\mathrm{e}}$ along $\bar{\Gamma}-\overline{\mathrm{M}}$. Subsequently, the same group reported results of a later experiment, ${ }^{74}$ where the binding energy was given as $0.54 \pm 0.30$ eV and, again, $\mathrm{m}^{*}=1.4$ along $\bar{\Gamma}-\overline{\mathrm{M}}$. Straub and Himpsel ${ }^{66}$ measured the binding energy as $0.77 \mathrm{eV}$ but reported no effective mass. Adsorbate studies by inverse photoemission have also been reported. In these cases, unoccupied orbitals of molecular species are typically observed and angle-resolved ( $k$-resolved) data are often not collected. Work on Ag(111) with adsorbed material is of particular importance to the present study. otto and coworkers examined pyridine ${ }^{75}$ and pyrazine ${ }^{76}$ on $\mathrm{Ag}(111)$ and reported molecular negative ion affinity levels 
stabilized by the image interaction at the surface. Frank and coworkers $^{\pi}$ studied a series of adsorbed aromatic hydrocarbons on Ag(111), also observing features in the spectrum assigned to affinity levels. A variety of inverse photoemission studies which were not angle resolved and were concerned with systems besides Ag(111) have been reported. Clean $\mathrm{Cu}(100),{ }^{11,29} \mathrm{Au}(100)^{11}$ and $\mathrm{Ni}(111)^{78}$ all have image potential states which have been observed by inverse photoemission. Features assigned to bulk states have been observed on polycrystalline silver, but image potential states were not observable. ${ }^{12}$ Two studies of molecular adsorbate systems are that of Frank and coworkers, 9 who examined benzene and azabenzenes on $C u(111)$ and $A u(110)$ and that of otto and coworkers, ${ }^{80}$ who performed a similar study of pyridine on silver films produced by evaporation at low temperatures. Interest in such films was due to their ability to enhance Raman activity of adsorbed molecules. Memmel and coworkers $^{32}$ studied inverse photoemission of sodium on $\mathrm{Ni}(111)$. Unoccupied levels found in this study were viewed as modified image potential states using an extension of multiple reflection theory.

Besides the work on $\mathrm{Ag}(111)$ already mentioned, many $\mathrm{k}$ resolved studies of image potential states have been reported. of the articles where molecular adsorbates were mentioned, Reihl and coworkers ${ }^{81}$ studied Ag(110), claiming the image potential state was quenched by the addition of oxygen and 
hydrogen. In a different paper, ${ }^{14}$ the same group also described a study of $\mathrm{Ag}(100)$ and reported quenching of the image potential state by physisorption of water. Finally, Reihl ${ }^{82}$ has observed the image potential state in the presence of adsorbed xenon on $\mathrm{Ag}(100)$ and reported the dispersion was unchanged except for a rigid shift to lower energies, tracking the adsorbate-induced work function lowering. This article also described the quenching behavior of water and oxygen reported earlier.

The adsorbate systems of alkali metals on transition metal surfaces have also been studied by $k$-resolved experiments. Jacob and coworkers ${ }^{83}$ examined Ag(110) with submonolayer quantities of $K$ and $N a$. The energy of the adsorbate-induced level was found to shift with coverage. The dispersion relation obtained experimentally was well fit by an effective mass of $\mathrm{m}^{\star}=2.5 \mathrm{~m}_{\mathrm{e}}$. Dudde and coworkers performed $\mathrm{k}-$ resolved measurements on $\mathrm{Cu}(111)$ with $\mathrm{K}$ coverages up to one monolayer. Potassium forms a $(2 \times 2)$ adlayer in this system. Two adsorbate-induced features were observed and were not assigned as image potential states. They were dispersive, but no effective mass was reported.

Besides the (111) face of silver of interest in the present work, many other ciean metal surfaces have been studied by $k$-resolved inverse photoemission. $\mathrm{Cu}(100), 15,58,28,17$ $\mathrm{Cu}(111)^{28}$ and $\mathrm{Cu}(110)^{30}$ have been examined. Experiments on $\mathrm{Ag}(100)$ were reported by Garcia and coworkers in the same 
article 64 in which they advanced their surface corrugation theory. In addition, $\mathrm{Ni}(100), 16,17 \mathrm{Ni}(110), 17 \mathrm{Ni}(111)^{84}$ and $\mathrm{Pd}(111)^{19}$ have also been studied by $\mathrm{k}$-resolved inverse photoemission. A large article $e^{66}$ by Straub and Himpsel reports the results of work on $\mathrm{Cu}(111), \mathrm{Cu}(100), \mathrm{Cu}(110), \mathrm{Ag}(111)$, $\mathrm{Sb}(100), \mathrm{Au}(111)$ and the $(5 \times 20)$ reconstructed face of $\mathrm{Au}(100)$. The authors point out that the image potential states are observable outside of gaps in the projected bulk band structure, using the case of $\mathrm{Au}(111)$ as an example. A useful table of measured binding energies and effective masses of image potential states on various surfaces is provideci in the article by Straub and Himpsel.

\subsubsection{Two-Photon Photoemission}

In two-photon photoemission, unoccupied surface states of a metal or semiconductor are populated by laser excitation from occupied levels lying below the Fermi energy. A second laser photon is then absorbed by the transiently occupied intermediate state, resulting in a photoelectron. The energy of the intermediate level is determined by analyzing the kinetic energy of the photoelectron. The intermediate state can be a bulk or surface state. Since these intermediate states are short-lived, the high peak intensity provided by laser excitation is essential. Instrumental energy resolution is limited by laser linewidth and the resolution of the photoelectron detector. Although the energy resolution of the 
detector is typically the more severe limitation, it is often nearly as narrow as the width of the transient levels themselves.

Dispersion information is obtained by reducing the photoelectron collection angle and systematically varying the angle of the detector axis relative to the surface normal. As in ordinary angle-resolved photoemission, ${ }^{21,85}$ the assumption is made that the parallel component of momentum of the state being probed is conserved upon excitation of a photoelectron. Then, given a photoelectron's measured kinetic energy (E) and its angle of emission relative to the surface normal $(\theta)$, the momentum of the intermediate state in the plane of the surface is

$$
k_{1}=\left(\frac{2 m_{0} E}{h^{2}}\right)^{1 / 2} \sin \theta
$$

Compared to inverse photoemission, two-photon photoemission offers much better energy resolution and the ability to time-resolve the decay of the intermediate state by ultrafast pump-and-probe techniques. It is better suited to studying materials with poor electrical conductivity since much lower electron currents are involved in the experiment. It is a less powerful tool for observing negative ion states of adsorbed molecules. The paucity of studies of molecular negative ions in the two-photon photoemission literature, 
compared to the inverse photoemission literature, is evidence for this conclusion.

The angle-resolved variant of two-photon photoemission is less able to observe states which have large values of momentum in the surface plane. In particular, interesting states near the surface Brillouin zone boundary may never be observable by two-photon photoemission. In any angle-resolved photoemission experiment, mechanical restrictions place an upper bound upon the angle which can be achieved between the detector axis and the surface normal of the sample. For a given rotation angle, the maximum value of momentum in the surface plane which can be sampled is Iimited by the perpendicular component of momentum which can be imparted to the photelectron. This can be understood simply by a vector addition argument. The perpendicular momentum is determined roughly by the kinetic energy of the photoelectron, and this is limited by the photon energy of the photoemitting light. since laser sources must be used in two-photon photoemission, the available photon energy has been limited to about $12 \mathrm{ev}$ or less. In their ability to probe large regions of the surface Brillouin zone, these experiments axe not competitive with single-photon photoemission experiments using noble gas resonance lamp sources or synchrotron radiation. Their capabilities in this regard may be linked in some degree to advances in laser technology.

Level energies obtained by the two different methods 
often do not agree exactly. This effect may be understood by noting that inverse photoemission probes an excess-electron state of an otherwise neutral system, whereas two-photon photoemission probes an electron which is correlated, in some degree, to the positive hole left behind in the substrate material. Only in the limit of instantaneous and complete screening of the positive hole by electronic charge in the substrate are the two techniques expected to give identical level energies.

The application of two-photon photoemission to image potential states has been reviewed in two articles. The first, ${ }^{8}$ by Giesen and coworkers, compares the method to second harmonic generation as a spectroscopic probe. The second, ${ }^{87}$ by Steinmann, emphasizes work on image potential states, including binding energy and dispersion measurements, as well as lifetime measurements reported in the literature. Bokor and Haight ${ }^{88}$ as well as Bokor ${ }^{89}$ (working alone) have written two recent reviews of two-photon photoemission and its application as a dynamical probe of unoccupied electronic states in metals and semiconductors.

Four two-photon photoemission studies of image potential states on Ag(111) exist in the literature. Three are by the group Giesen, Hage, Himpsel, Riess and Steinmann. The first 90 reports a binding energy of $0.77 \mathrm{eV}$ for the $n=1$ image potential state and $0.23 \mathrm{eV}$ for the $n=2$ state. The quoted resolution is $\pm 0.03 \mathrm{ev}$. Since this was not an angle-resolved 
study, no effective mass was measured, and only photoemission normal to the surface was collected. A wavelength dependent experiment in this study provided evidence that image potential states on the Ag(111) surface were undergoing three separate dynamical processes. The first process was a simple resonant excitation of an electron from the occupied L-gap surface state to the image potential state. The second process was excitation of initial bulk states above the projected bulk band gap, followed by popu'ation of the image potential state due to energy relaxation of electrons in the bulk states. The third was inelastic collision between two image potential states, with one being ejected into the vacuurn and the other decaying to an unoccupied part of the surface state band.

The second paper ${ }^{65}$ is intended chiefly as an experimental refutation of the corrugation theory of Garcia and coworkers. ${ }^{4}$ An effect predicted to be prominent by the corrugation theory was a strong dependence of image potential state binding energy upon the effective mass of the electron. By collecting angle-resolved two-photon photoemission data on image potential states on the $\mathrm{Ag}(111), \mathrm{Cu}(111)$ and $\mathrm{Ni}(111)$ surfaces, Giesen and coworkers found the relationship between binding energy and effective mass to be at least a factor of 10 too weak to support the corrugation model. For the $n=1$ state on Ag(111), in particular, the $n=1$ image state was reported to be bound by $0.77 \pm 0.03 \mathrm{eV}$ and had an effective mass of 
$(1.35 \pm 0.15) \mathrm{m}_{e}$. The orientation in the surface Brillouin zone along which this dispersion was measured was not specified.

In making the angle-resolved measurement, the authors excited initial states in the continuum of bulk bands below the Fermi level. The importance of exciting from bulk states rather than surface states when attempting to measure the dispersion relation of the image potential state reliably by two-photon photoemission was emphasized explicitly in a later paper by Steinmann. ${ }^{87}$ Data on $\mathrm{Cu}(111)$ and $\mathrm{Ni}(111)$ were also presented in the second paper.

In the third article dealing with the image potential states on Ag(111), 50 Giesen and coworkers, along with Smith, presented a detailed treatment of the effect of the projected bulk bands upon the image potential states. Both the binding energy and the dispersion relation were treated. Multiple reflection theory was adapted to this end, and its predictions were compared to dispersion curves measured by angle-resolved two-photon photoemission. Experimental values for the $n=1$ state on $\mathrm{Ag}(111)$ were a binding energy equal to $0.77 \pm 0.03 \mathrm{eV}$ and an effective mass of $\mathrm{m}^{*}=(1.30 \pm 0.15) \mathrm{m}_{\mathrm{e}}$. The orientation in the surface Brillouin zone along which the dispersion was measured was not given. The experimental values were compared to the predictions of multiple reflection theory, which were a binding energy of $0.75 \mathrm{eV}$ and an effective mass of $1.33 \mathrm{~m}_{\mathrm{e}}$. Similarly calculated theoretical values for Ag(100) and Cu(100), which were also given in this article, do not fall 
within the boundary of the quite narrow experimental error bars. Considering the simplicity of the model based upon multiple reflection theory, however, its agreement with experiment is tolerable.

The fourth study of image potential states on Ag(111) is by schoenlein and coworkers ${ }^{91}$ and is a lifetime measurement done by ultrafast pump-probe techniques. The lifetime of both the $n=1$ and $n=2$ states on $A g(111)$ was determined to be less than 20 fs. This very short lifetime was attributed to the image potential states' location relative to the projected bulk band gap on this surface. The $n=1$ state is just below the upper band edge, and the $n=2$ is actually above it. Thus, the oscillatory tails of the image potential state's wave functions extend a relatively long distance into the bulk, and coupling with bulk states is expected to be large. Image potential states on the (100) surface were markedly different.

Several two-photon photoemission studies mention the effects of adsorbates upon image potential states, though none of these deal with the Ag(111) surface. In an early paper by Rudolf and Steinmann, 92 excitation of two-photon photoelectrons was first reported. No photoelectron energy analysis was performed in this work. A charge transfer state of $\mathrm{CO}$ on $\mathrm{Ni}(111)$ and $\mathrm{Ni}(110)$ was allegedly observed. Study of this adsorbate system was not pursued in subsequent work. Rieger and coworkers ${ }^{93}$ examined oxygen chemisorbed on $\mathrm{Cu}(111)$. The $\mathrm{n}=1$ image potential state, clearly observable on 
the clean surface, was entirely quenched by a saturation coverage of 0.3 monolayers of oxygen. Two adsorbate-induced features appeared. A state below the Fermi energy was excited by nonresonant two-photon photoemission, and a separate feature assigned as an unoccupied state was observed as an intermediate level. The two different excitation mechanisms were elucidated by wavelength-dependent experiments.

Hamza and Kubiak, 40 in their study of image potential states on $\mathrm{Ni}(111)$, observed the effect of chemisorbed oxygen and noted the disappearance of a weak, broad peak in the photoelectron kinetic energy distribution that was not dispersive. It was attributed to nonresonant two-photon photoemission from a state below the Fermi energy.

In a high resolution two-photon photoemission study, Fischer and coworkers ${ }^{94}$ reported a narrowing of the linewidths of the first three image potential levels on $\mathrm{Ni}(111)$ when the sample was exposed for an hour to the hydrogen-rich background gases in the ultra-high vacuum chamber. It was suggested that the contamination produced a quenching of other surface states. These states, when present, could act as decay channels for the image potential state, so that the increased lifetime of the image potential state in the presence of adsorbate directly produced the observed reduction in linewidth. The same group later studied monolayer sodium on $\mathrm{Cu}(111)$ and observed a series of three image potential states near the vacuum level. Their energies 
were reported to agree qualitatively with the predictions of the modified multiple reflection theory of Iindgren and Walldén ${ }^{34}$.

Aside from the work on adsorbates and Ag(111), two-photon photoemission studies of other clean metal surfaces are plentiful. A very early paper by Rudolf, Rieger and steinmann 95 reports two-photon photoemission from $N i(110)$ and Ni(111). Although photoelectron energies were not analyzed, a quadratic dependence of total photoelectron yield upon laser power as well as a strong dependence of yield upon laser polarization were observed. Much more recently, schuppler and coworkers $^{36}$ measured the binding energies of the first three members of the image potential state series on $\mathrm{Ni}(111)$. The effective mass of the $n=1$ state was measured and the resonant excitation from the occupied L-gap surface state to the $n=1$ image potential state was tracked out to $0.2 \AA^{-1}$ in the surface Brillouin zone by varying the laser wavelength. Kubiak ${ }^{30}$ studied $c u(111)$, measuring the binding energies and effective masses of the $n=1$ and $n=2$ image potential states and comparing these results to multiple reflection theory. on $\mathrm{Cu}(100)$, the bulk band structure was probed by two-photon photoemission in the experiments of wegehaupt and coworkers.96 According to the authors, an electron was initially excited from the highest $d$-band at $x$ in the bulk Brillouin zone to the unoccupied state at the edge of the first free-electron-like band. The final state of the excitation was claimed to be an 
evanescent state just above the vacuum level in the bandgap at $x$. If these assignments are correct, this paper is evidence that two-photon photoemission is a useful, very precise probe of bulk electronic states as well as surface states. Kubiak ${ }^{38}$ performed two-photon photoemission on Pd(111), detecting both a bulk state and the $n=1$ image potential state. Giesen and coworkers ${ }^{35}$ have measured the binding energies of the $n=1$ and $\mathrm{n}=2$ image potential states on $\mathrm{Ag}(100)$ and $\mathrm{Cu}(100)$ using high energy resolution $( \pm 0.020 \mathrm{eV})$. The utility and predictive power of multiple reflection theory for determining eigenvalues of image potential states on various metals and crystallographic faces was again demonstrated when calculated and experimental values obtained in this study were compared.

In the experiments of Schoenlein and coworkers, the decay dynamics of image potential states were directly time-resolved by two-photon photoemission with ultrafast lasers. Initially, the $n=1$ state on $A g(100)$ was studied.97.98 Its lifetime was short compared even with the 50 fs laser pulse, but was bracketed between 15 and 35 fs. A latex article ${ }^{91}$ reports the measurement of the lifetime of the $n=2$ state on $A g(100)$ as 180 fs. This ratio of lifetimes compares quite well with the factor of 8 predicted by de Andres and coworkers using the self-energy formalism. ${ }^{54}$ This is the same article in which lifetimes under 20 fs for $n=1$ and 2 states on $A g(111)$ were reported. The difference in behavior between the two faces of the same metal is striking, and is taken in the article as an 
indication of the dramatic influence that projected bulk bands can exert upon the image potential states.

In addition to its application to image potential states, two-photon photoemission has been used to obtain important dynamical information on the relaxation of excited electronic states of semiconductors. The first application of this technique to such a problem was by Williams and coworkers, 99 who studied $z$ nTe, observing a 150 ps relaxation of photoexcited hot carriers to the conduction band minimum and a 300 ps decay of the relaxed state. Haight and coworkers ${ }^{100,109}$ studied decay dynamics of excited electrons in a normally unoccupied surface band and also measured the dispersion of this band. Haight and Bokor ${ }^{102}$ later studied p-type doped GaAs(110), again time-resolving the decay of surface states. Haight and Silbermann ${ }^{103}$ observed intervalley scattering among excited surface states on GaAs(110). The same researchers also studied the system of Ge grown epitaxially on GaAs(110). A normally unoccupied state derived from the $G e$ layer was observed. Bokor and coworkers $^{104}$ examined the decay dynamics of the unoccupied antibonding surface state on $S i(111)(2 \times 1)$. Later, Halas and Bokor ${ }^{105}$ studied the dynamics of surface recombination of bulk electron-hole pairs on the same surface. Kubiak and Kolasinski ${ }^{106}$ measured the dispersion of an unoccupied surface state on the (111) (2×1) surface of diamond. 
The present two-photon photoemission study relies upon existing theoretical and experimental studies of the bulk bands of silver and the electronic structure of the Ag(111) surface. Knowledge of the information summarized here has guided both the design of the experiment and the interpretation of the results.

Silver crystallizes in a face-centered cubic unit cell with a lattice constant of $4.09 \AA .45$ This results in a nearest-neighbor distance on the (111) face of 2.89 A. Atoms in this face are arranged in a hexagonal array. The dimensions of the bulk Brillouin zone are $1.53 \AA^{-1}$ from $\Gamma$ to $\mathrm{X}\left((100)\right.$ direction) and $1.33 \mathrm{~A}^{-1}$ from $\Gamma$ to $L$ ( (111) direction). The center of the surface Brillouin zone, where the crystal momentum in the plane of the surface $\left(k_{1}\right)$ is equal to zero, is denoted by $\bar{\Gamma}$. The (111) surface Brillouin zone ${ }^{21}$ is a regular hexagon, and its dimension from the origin to the midpoint of a side (that is, $\bar{\Gamma}-\bar{M}$ or $\bar{\Gamma}-\bar{M}^{\prime}$ ) is $1.26 \mathrm{~A}^{-1}$. Its dimension from the origin to a vertex $(\bar{\Gamma}-\bar{K}) \quad 1.45 \AA^{-1}$. The placement and orientation of the surface Brillouin zone over the bulk Brillouin zone is depicted to scale in Figure 8 . The bulk symmetry points in this figure are located in $\mathrm{k}$-space as follows:

$$
\begin{aligned}
& K=(3 / 4,3 / 4,0) \cdot 2 \pi / a \\
& L=(1.2,1 / 2,1 / 2) \cdot 2 \pi / a \\
& W=(1,1 / 2,0) \cdot 2 \pi / a
\end{aligned}
$$


Caption for Figure 8:

a) Bulk FCC Brillouin zone with zone center and points of high symmetry labelled.

b) (111) surface Brillouin zone for FCC lattice with zone center and symmetry points labelled.

c) Bulk and surface Brillouin zones (a) and (b) overlaid in correct orientation in reciprocal space. $\bar{\Gamma}-\bar{M}$ and $\bar{\Gamma}-\bar{M}^{\prime}$ are equal to $1.26 \mathrm{~A}^{-1}$, and $\bar{\Gamma}-\bar{K}$ is equal to $1.45 \AA^{-1}$ for silver. 
a)

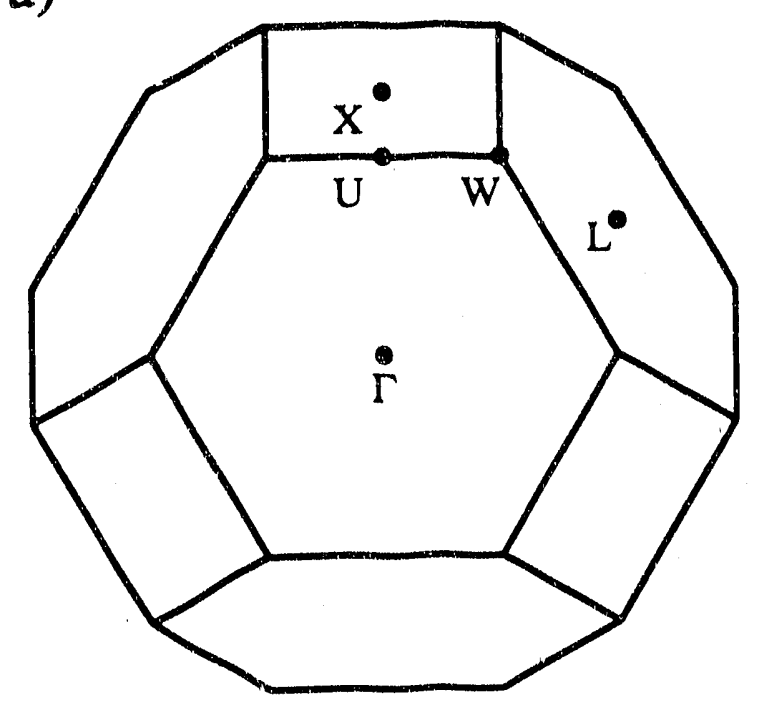

b)

c)

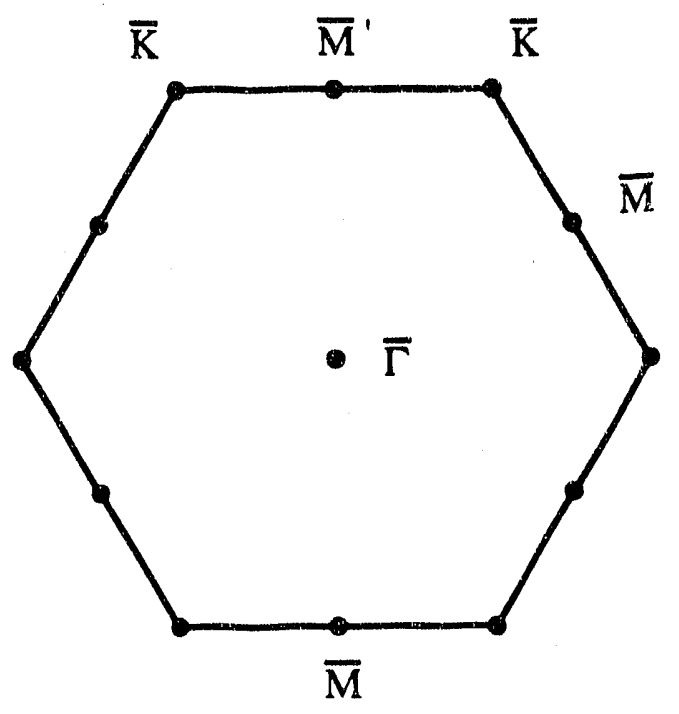

Figure 8

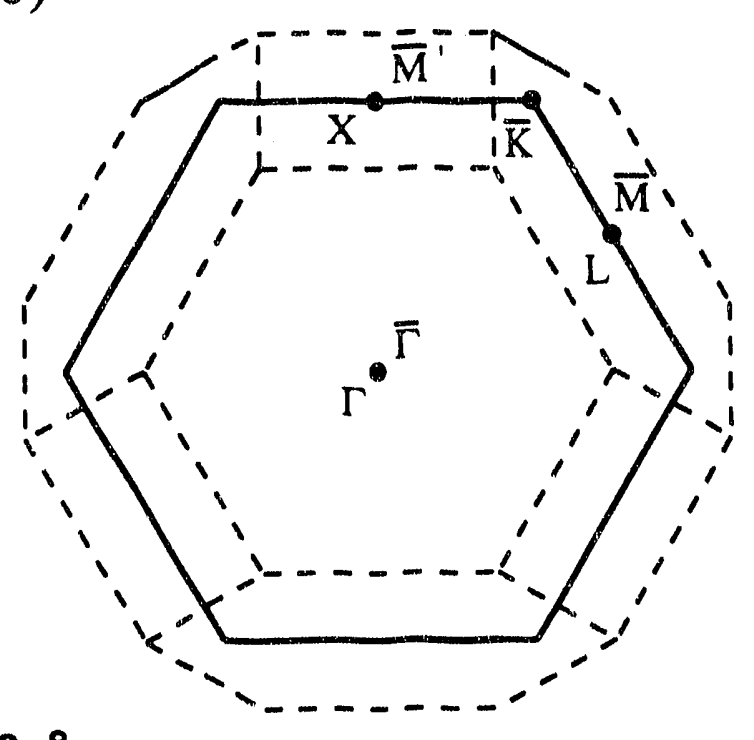




$$
\begin{aligned}
& X=(1,0,0) \cdot 2 \pi / a \\
& U=(1,1 / 4,1 / 4) \cdot 2 \pi / a
\end{aligned}
$$

Here, a is the lattice constant in direct space. A useful and thorough discussion of Brillouin zone geometry for various lattices is the article by Eberhardt and Plummer. ${ }^{21}$

As all noble metals do, silver has both d-bands and $s-p$ bands.113,114,107 Its calculated bulk band structure ${ }^{107}$ is represented in Figure 9. It is a very useful oversimplification to treat the $s-p$ bands as though they were those of free electrons. Occupied d-bands lie between 4 and $8 \mathrm{eV}$ below the Fermi energy and are relatively flat. The origin of the s-p (free-electron like) band structure is about $4.5 \mathrm{ev}$ below the Fermi energy, and this dispersive set of bands marches upwards from there.

Although the net of (11.1) surface atoms is sixfold symmetric, the two-dimensional band structure in the surface plane is, strictly speaking, only three-fold symmetric due to coupling to the lattice. Thus, diametrically opposite sides of the surface Brillouin zone are not strictly equivalent. In the literature, a distinction is made between points $\bar{M}$ and $\bar{M}$ ', located at the midpoints of the surface Brillouin zone sides. Conventionally, $\bar{M}$ is aligned to lie directly over a bulk $L$ point (the center of a hexagonal face of the bulk Brillouin zone), and $\bar{M}$, is aligned to lie directly over a bulk $x$ point (the center of a square face of the bulk Brillouin zone). Midpoints of the surface Brillouin zone alternate in identity 
Caption for Figure 9:

Calculated bulk band structure of silver. This figure was adopted in a nearly quantitative way from the article of Exkhardt, Fritsche and Noffke (reference 107). The free-electron s-p derived bands are those crossing the Fermi energy and those lying above it. Here the gap at is spans the energy range from about 0 to $3.5 \mathrm{eV}$. It is thus slightly narrower than is observed experimentally. The line $L-W$ in the bulk is parallel to that from $\bar{\Gamma}$ to $\bar{K}$ in the (111) surface Brillouin zone. The band edges defining the gap disperse rapidly upward and the gap closes as either of these lines is traversed. 


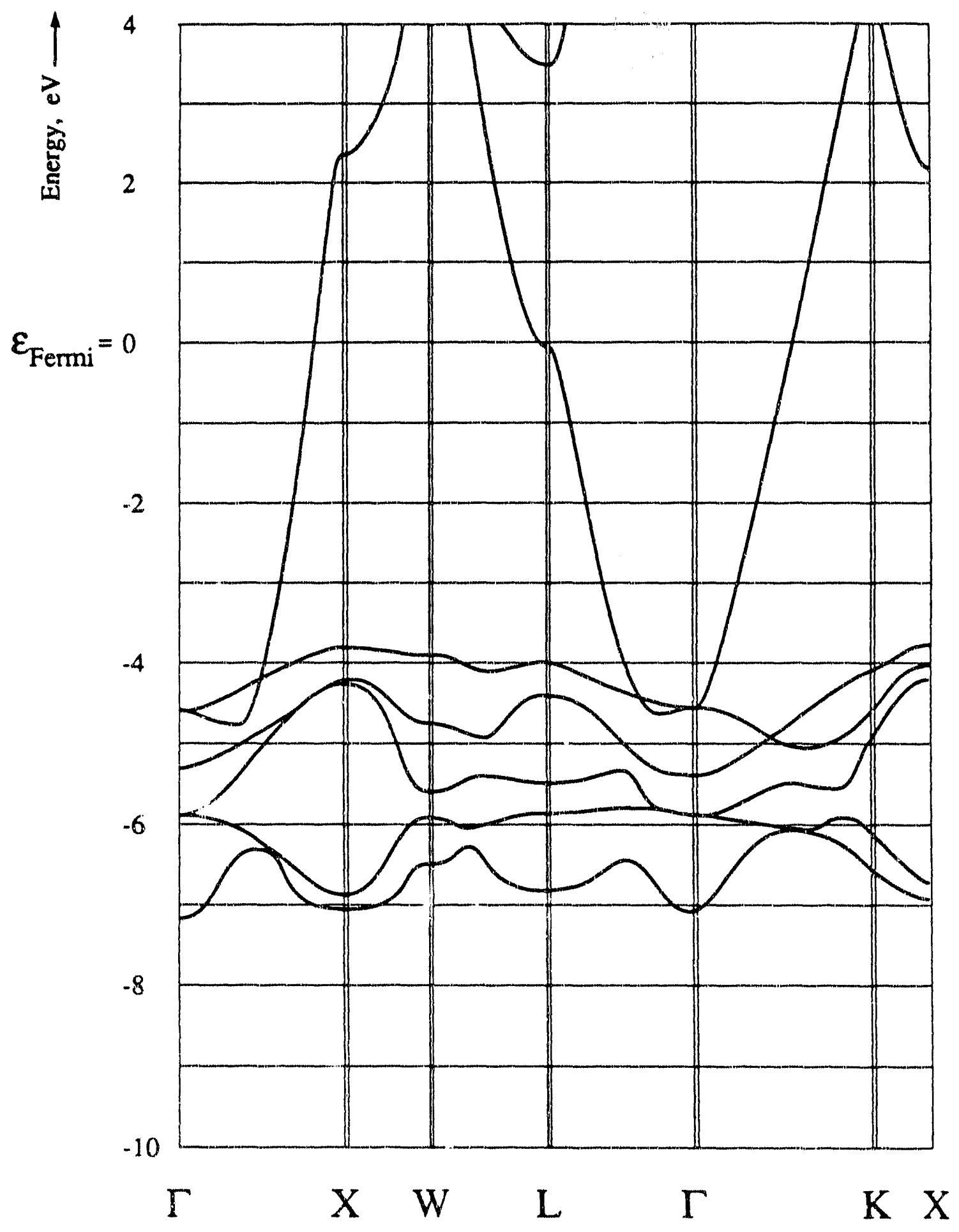

Figure $\quad$ Bulk wave vector between various symmetry points in Brillouin zone 
between $\bar{M}$ and $\bar{M}^{\prime}$. All six vertices of the surface Brillouin zone ( $\bar{K}$ points), however, are taken in the literature as equivalent.

Orientation of the surface Brillouin zone is such that each of the three lines $\bar{\Gamma}-\bar{M} \cdot$ is parallel to one of the three bulk lattice vectors which are permutations of the Miller indices $(2, \bar{I}, \bar{I})$. Each of the three lines $\bar{\Gamma}-\bar{M}$ is parallel to one of the three bulk lattice vectors which are permutations of $(\overline{2}, 1,1)$. Each of the six $\bar{\Gamma}-\bar{K}$ lines is parallel to one of the six bulk lattice vectors which are combinations of the indices $(\overline{1}, 1,0)$.

The gap which occurs between the first and second freesiectron-like bulk bands of silver and is centered on the bulk I point is due to the well-known necks in the Fermi surface, which touch the Brillouin zone boundary, forming a small circle about $I$. Within the perimeter of this circle, bulk states do not cross the Fermi level. The fact that the crosssection of the Fermi surface neck is a circle, rather than some shape of lower symmetry, is strong evidence that the bulk band edges forming the gap are azimuthally symmetric about. the $\Gamma-I$ Iine. In other words, the curvatures of the upper and lower band edges are largely independent of the direction in which these curvatures are measured inside the gap.

This band gap is often referred to as the I-gap. Working with a calculated band structure, Ho and Piazzalos have projected this gap into the (111) plane in reciprocal space. 
Unfortunately, important details of their calculation are lacking in the article. The upper and lower band edges which define the gap both disperse upward, so that the gap extends from below the Fermi energy at $k_{1}=0$ to above the vacuum enexgy near the boundary of the surface Brillouin zone.

The projected bulk L-gap supports both the image potential states studied in the present research and the occupied surface state of $\Lambda_{1}$ symmetry discovered by Gartland and slagsvold.90,86,109 This gap is $s-p$ inverted, so that the standing-wave bulk wave functions of electrons whose energies are at the upper band edge are s-like funtions, while those at the lower band edge are are $p-1 i k e .31,20$

The bulk electronic wavefunction of the lower bulk band edge at the center of the surface Brillouin zone is referred to as the $L_{2}$, state. Its energy was determined experimentally by high resolution angle-resolved ultraviolet photoelectron spectroscopy. 110 It was reported to be $-0.30 \pm 0.02 \mathrm{eV}$ relative to the Fermi energy. The dispersion of the lower projected bulk band edge used in the present study was taken from the work of smith and coworkers ${ }^{50}$ and is $m^{*}=0.60 \mathrm{~m}_{e}$. The orientation in the surface Brillouin zone for which this dispersion was calculated was not given in smith's paper. References for the dispersion figure were not given either, although smith has described a method based on interpolation from the calculated bulk band structure in a different article. ${ }^{17}$ It is of use to note that the crossing point of the 
Fermi energy and the lower projected bulk band edge occurs at a value of momentum in the plane of the surface which is equal to the radius of the Fermi surface neck about $L_{\text {. }}$. This radius has been measured experimentall $y^{191}$ as $0.164 \mathrm{~A}^{-1}$.

The energy of the upper band edge at the center of the surface Brillouin zone (the $L_{1}$ state) was measured accurately by angle-resolved two-photon photoemission.90,86 It was given as $3.85 \mathrm{eV}$ relative to the Fermi energy. The dispersion of the upper bulk band edge from smith's work ${ }^{50}$ was $m^{*}=1.60 m_{e}$. The fact that smith fails to report the direction in the surface Brillouin zone along which these band edge dispersions are specified is somewhat ameliorated by the high symmetry of the bulk band edges about the $I$ point. One could expect to see very similar dispersions of the band edges when viewed along the $\bar{\Gamma}-\bar{M} \cdot, \bar{\Gamma}-\bar{M}$ or $\bar{\Gamma}-\bar{K}$ directions.

An independent measurement of the energy difference between $I_{4}$ and $L_{L}$, was made by chen and segall $1^{112}$ using an experimentally obtained piezooptical response function corresponding to transitions between these two states in the bulk. Their value of $4.20 \mathrm{eV}$ compared well with the value of $4.15 \mathrm{eV}$ derived from the parameters obtained by ultraviolet photoemission and two-photon photoemission.

The energy and dispersion of the $\Lambda_{1}$ surface state were also measured by ultraviolet photoelectron spectroscopy. ${ }^{110}$ At the center of the surface Brillouin zone, its energy was $-0.12 \pm 0.01 \mathrm{eV}$ relative to the Fermi energy. It was 
characterized by an effective mass of $\mathrm{m}^{*}=(0.53 \pm 0.01) \mathrm{m}_{e}$. The orientation in the surface Brillouin zone along which the dispersion was measured was not given.

The binding energy of the $n=1$ image potential state on Ag(111) determined by two-photon photoemission $90,65,50$ and invers photoemission. ${ }^{27,31,66}$ For the purposes of the present experiment, the best value from the literature was judged to be $0.77 \mathrm{ev} .90$ The effective mass of the $n=1$ image potential state was determined by two-photon photoemission 65,50 and inverse photoemission. ${ }^{27,31,66}$ The best value was judged to be $\mathrm{m}^{*}=(1.30 \pm 0.15) \mathrm{m}_{e^{\cdot}}{ }^{50}$ The orientation in the surface Brillouin zone was not given in this reference. The binding energy of the $n=2$ image potential state was reported in the literature as $0.23 \mathrm{ev} .90$ No effective mass for the $n=2$ state was reported in the literature. The best value for the work function of Ag(111) was judged to be $4.56 \mathrm{ev}$, the value of steinmann. ${ }^{86}$ The bulk and surface electronic states of the Ag(111) L-gap described here are also diagrammed in Figure 10.

Four theoretical calculations of the band structure of silver have been important to the present work. The earliest was due to christensen ${ }^{113}$ and was the first relativistic band structure calculation for this material. A later calculation was reported by Jepsen, Glötzel and Mackintosh. ${ }^{114}$ This paper gives a useful summary and comparison of experimental and calculated values for the width of the L-gap. The more recent work of Eckhardt and coworkers ${ }^{107}$ treats in some detail 


\begin{abstract}
Caption for Figure 10:
Projected bulk band structure of silver (111) drawn to scale, along with two members of image potential state series. The $n=0$ image potential state is also referred to as the L-gap state or simply the occupied surface state.
\end{abstract}




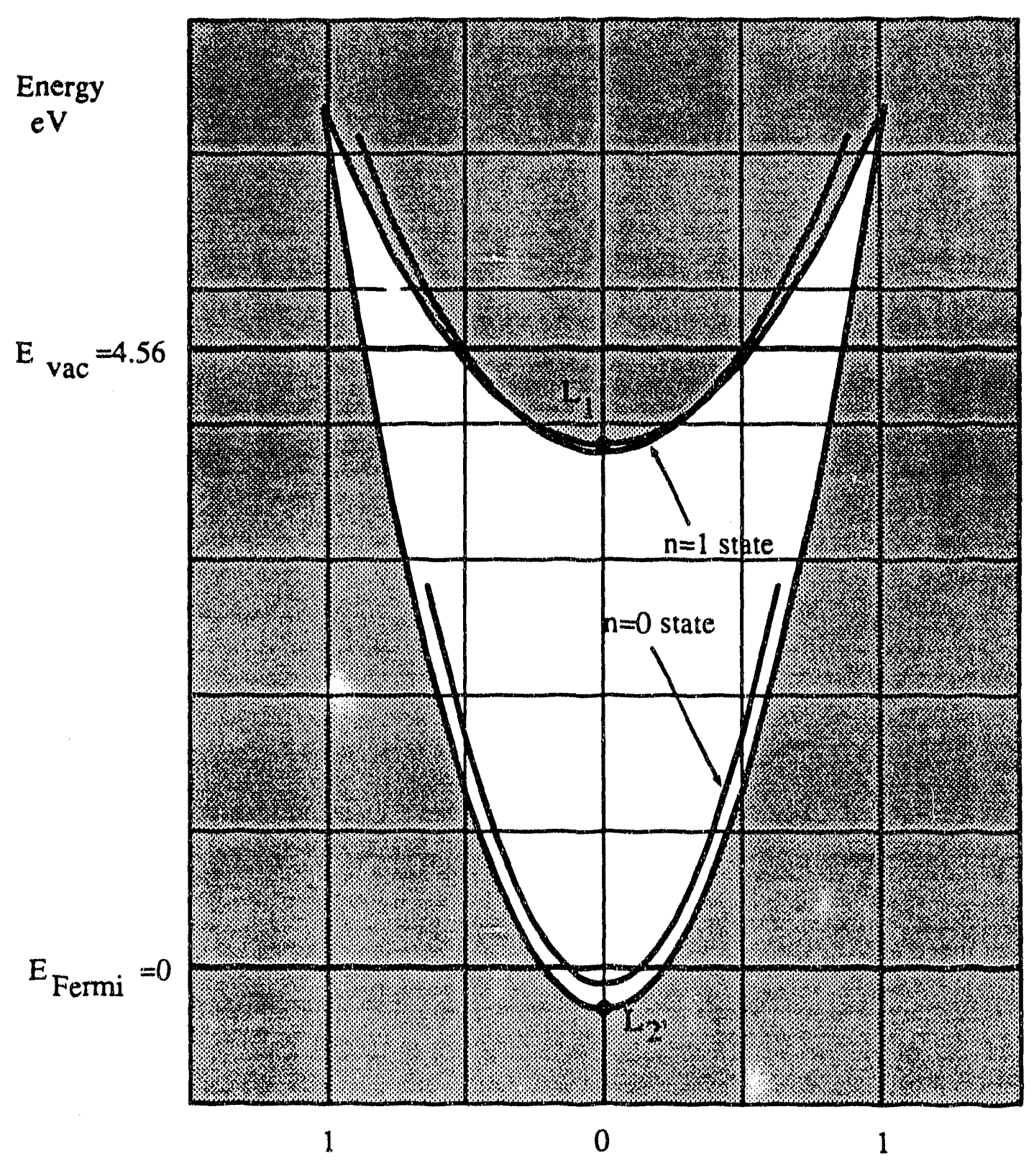

wave vector in plane of surface

$$
k_{11}, \AA^{-1}
$$

Figure 10 
the radius of the Fermi surface necks at $L$.

Band mapping studies by angle-resolved photoemission provide accurate, reliable information of the bulk band structure. The earliest of these was by Liebowitz and Shevchik. ${ }^{195}$ subsequent studies have been reported by wehner and coworkers, ${ }^{116}$ spears and coworkers, ${ }^{117}$ and courths and coworkers.118 The optical reflection and absorption properties of silver in the visible and near ultraviolet regions of the spectrum have been reported by Ehrenreich and Philipp. 119 A useful review article of experimental and theoretical work concerning electronic properties of many metals including silver has been written by visscher and Falicov. ${ }^{120}$ 
A number of requirements must be met by the apparatus in order to study in a controlled fashion image potential states on metal surfaces and their interactions with thin adsorbed layexs of material. In order to guarantee that the surface supporting the image potential states is adequately free from contamination, standard ultra-high vacuum techniques must be used. The sample holder must be constructed of nonmagnetic, high-temperature and ultra-high vacuum compatible materials. It must also offer precise angular motion and provide heating, cryogenic cooling, and measurement of sample temperature. samples must be single crystals and have physically and chemically well characterized, atomically smooth surfaces. The laser ased as an excitation source must be tunable and provide subnanosecond pulses in order for time-of-flight energy analysis to perform optimally. Pulse energies cannot be large enough to distort the photoelectron spectra by space charge effects. The time-of-flight technique itself depends upon a fast electron multiplier and effective electromagnetic shielding of the flight region. Knowledge of the contact potential difference existing between the sample and the detector is crucial for time-of-flight analysis to succeed, particularly when the sample's work function is deliberately changed by deposition of adsorbed material. This section is 
a detailed account of our approach to the many practical requirements of this experiment.

\subsection{Ultra-High Vacuum system}

\subsubsection{Original Chamber}

Two different ultra-high vacuum chambers were used to collect the data with which this experiment is concerned. Experiments in which the sample was cooled below about $60 \mathrm{~K}$ were performed in the chamber originally built by $A$. Gal10.129 In this case, the sample was anchored by vacuum brazing with cusil alloy onto a machined block of type 310 stainless steel. By the use of a specially designed manipulator, the sample could be inserted into and removed from a copper sample holder rigidly mounted directly at the end of an ultra-high vacuum compatible Air Products LT-3B-110 continuous flow liquid helium cryostat, referred to as the cold tip. Sample temperature was measured with a silicon diode sensor. The threshold for thermal damage to the silicon diode was about $150 \mathrm{C}$ and was thus the highest temperature the sample could reach in the sample holder. The sample holder was electrically grounded to the chamber itself. In the Gallo chamber, sputterring and annealing of the sample were performed with the sample removed from the cold tip. Argon sputter gas could be continually flowed through the chamber and evacuated, at certain times with a turbomolecular pump and at other times with a liquid nitrogen trapped diffusion pump. 
These pumps were also used for roughing the chamber down from atmospheric pressure after it was vented. Annealing of the sample was provided by electron bombardment heating in a dedicated high voltage annealing stage. Sample temperature was measured with a chromel-alumel thermocouple and an electrically floating, battery-powered digital voltmeter. Adsorbate gases and sputtering argon were introduced into the ultra-high vacuum chamber through a Varian leak valve from a diffusion pumped high vacuum manifold.

The main pumps on the ultra-high vacuum chamber were a Varian $400 \mathrm{~L} / \mathrm{s}$ triode ion pump and a Varian Ti-Ball titanium sublimator with a water-cooled jacket. After pumpdown from atmosphere, the chamber typically was baked at an air temperature of $150 \mathrm{c}$ overnight, completely enclosed in a freestanding oven made from asbestos and sheet metal. A base pressure of $3 \times 10^{-11}$ torr was routinely achieved. Residual gas analysis and helium leak testing were accomplished using a UTI $100 C$ quadrupole mass spectrometer. Varian 981-0127 four-grid LEED optics and controlling electronics were used in verifying atomic-scale order of the sample surfaces and in diagnosing surface contamination by Auger spectrometry.

This chamber had several inherent shortcomings in its application to the present research, and they eventually caused it to be replaced. Although the sample was mounted in such a way as to preclude rotation for angle-resolved experiments, it was not mounted rigidly. Thus, the length of 
the photoelectron flight path, an essential parameter in timeof-flight analysis, was known only to an accuracy of a few per cent. The time-of-flight analyzer and LEED optics both required the same port in the chamber and thus could not be used at the same time. Venting and bakeout was necessary between LEED/Auger analysis and two-photon photoemission experiments. No valve was provided for isolation of the ion pump and titanium sublimator region during sputtering, introduction of adsorbate gases, or venting. The ion pump used four large magnets which produced a detectable magnetic field at the position of the sample. The high vacuum roughing was accomplished through a port of 1.38 inch inside diameter, which had less than $10 \mathrm{~L} / \mathrm{s}$ conductance, a significant limitation to pumping speed. on the whole, the only advantage to this system was its capability of near-optimal thermal contact between the sample and the cold tip.

\subsubsection{New Chamber}

A new chamber was built for angle-resolved two-photon photoemission studies of image potential states in the presence of adsorbed layers. A plan view of this chamber appears in Figure 11. A custom stainless steel bell jar provides three 8-inch $O . D$. ports for a window, a time-offlight photoelectron energy analyzer, and LEED optics (recycled from the old chamber). Six 6-inch O.D. ports are also provided for a leak valve with line-of-sight sample 


Caption for Figure 11 :
plan view of the ultra-high vacuum chamber
designed for angle-resolved two-photon
photoemission experiments of surfaces with
physisorbed overlayers. At the top of the
manipulator, the micrometer operating the secondary
rotation is mounted eccentrically. The drawing is
approximately $1 / 10$ scale. Dimensions taken from
this drawing should not be used for design
purposes.




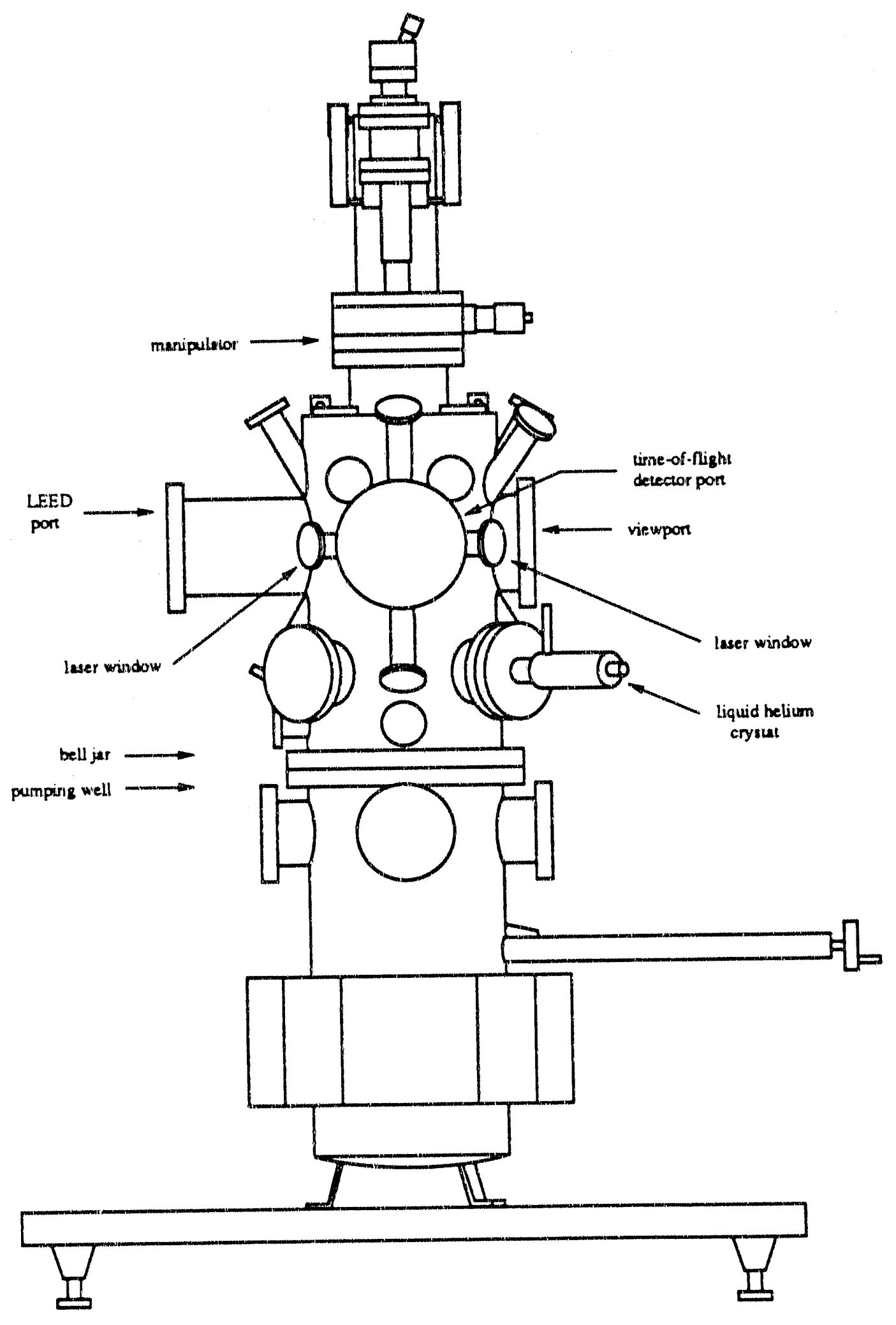

Pigure 12 
dosing nozzle, a Kelvin probe ${ }^{122}$ for work function measurements, a quadrupole mass spectrometer (recycled from the old chamber), as well as a viewport to observe the sample when positioned in front of any of these instruments. A precisely aligned 8 inch port on top of the bell jar supports the sample manipulator. Below the main instrumentation ports, foux 6 inch O.D. ports are located at positions to conveniently accomodate a liquid helium flow cryostat. This cryostat is connected to the sample holder module on the manipulator by a flexible copper rope. Four welded lifting eyes at the top of the bell jar are intended for lifting it independently of the pumping well below it and are not recommended for use in lifting the assembled chamber. A standard 14.63 inch $O . D$. female wire seal flange at the base of the bell jar mates with the top of the pumping well. A variety of 2.75 inch $O . D$. ports are provided for windows, valves, feedthroughs and instruments.

The pumping well is a customized Perkin-Elmer model TNB-X unit. It comprises the lower part of the ultra-high vacuum vessel. A 14.63 inch O.D. male wire seal flange at the top of it connects it to the bell jar. Four 6-inch $0 . D$. ports at the sides are intended to provide high conductance to an external high vacuum pump. Four 2.75-inch 0. D. ports are provided for gauging and feedthroughs. A viton sealed poppet valve actuated by a vertical jack and rotary feedthrough mechanism isolates the chamber and the upper region of the pumping well 
from the ion pump and titanium sublimator at the bottom. The poppet valve is typically closed for sputtering, sample dosing, and venting of the chamber. The differential type ion pump has a $300 \mathrm{~L} / \mathrm{s}$ rated pumping speed for nitrogen and consists of eight separate ion pumping cells arranged radially around the perimeter of the vacuum vessel. Because of the high symmetry with which these cells are placed, the strong permananent magnets between them efficiently cancel one another's magnetic fringe fields only a short distance away. At the plane of the wire seal flange, residual field strength due to the ion pump is less than 10 milligauss. This ion pump is thus particularly compatible with the low-field requirements of photoelectron energy analysis. In routine operation, this pump remains on continually. The titanium sublimator consists of four separate filaments of titaniummolybdenum alloy which are resistively heated to temperatures at which macroscopic amounts of the metal are able to sublime. only one of the filaments is sublimed at any one time, and they are thus consumed sequentially. sublimed metal is deposited on a panel cooled by continuously flowing water. In routine operation, sublimation of material is controlled by a timer and occurs for two minutes every few hours. The typical base pressure of the chamber is $1 \times 10^{-10}$ torr.

The chamber is supported at the base of the pumping well by three feet which are bolted to a massive, flat aluminum plate. Four inverted screw jacks are attached to the bottom 
of this base plate to enable accurate leveling of the chamber. An array of 1/4-20 tapped holes on 4 inch centers in the top of the plate enable cables and water lines to be routed and fastened down. They also anchor an aluminum and wood tabletop which surrounds the chamber at a convenient working height. This structure is designed for rapid disassembly prior to system bakeout. The base is attached to the floor with brackets and concrete anchors for safety in the event of an earthquake.

High vacuum pumping independent of the ion pump and sublimator is provided by an Edwards $200 \mathrm{~L} / \mathrm{s}$ turbomolecular pump. This pump is not able to be baked and reaches an ultimate vacuum in the mid $10^{-8}$ torr range when backed at 10 millitorr or less. Its chief wearing parts are the rotor bearings which, according to the manufacturers, have a lifetime of two years in continuous operation before they must be replaced. They should be relubricated at 6 month intervals during this lifetime. This pump has a 6 inch 0.0 . conflat type intake port. It is manually isolated from the chamber by an ultra-high vacuum compatible butterfly valve with a viton seat seal and an all-metal bonnet seal. When this valve isolates the chamber from atmospheric pressure, its leak rate due to diffusion of gas through the viton seal is high enough to keep the chamber in the $10^{-7}$ torr range with the ion pump runing. The turbomolecular pump assembly is bolted to the floor independently of the chamber. The two are connected by 
a stainless steel formed bellows which provides strain relief between them. Two adjustable bracing rods support the bellows against atmospheric pressure (an axial load of about 400 pounds) when the chamber is evacuated.

The turbomolecular pump is backed by a Balzers two-stage direct-drive rotary vane pump with a rated pumping speed of 12 cubic feet per minute. Two mist and vapor filters have been placed between this pump and the turbomolecular pump. oil mist first encounters a wire mesh baffle trap. It is hoped that any remaining vapors are captured by a molecular sieve trap, which may be regenerated when necessary by baking it with an internal heater. The rotary vane pump contains a generator coupled to its shaft which supplies a 3 volt AC output signal when it is operating. This signal is used in the interlock system described below.

In an effort to guarantee non-disastrous shutdown of the turbomolecular pump in the event of mechanical or power failures, two pneumatic isolating valves and a solenoid vent valve are interlocked with the logic interface of the turbomolecular pump controller. This logic interface senses and indicates the absence or presence of normal system operation. When the system is operating normally and the interlocks for automatic shutdown are armed, the automatic shutdown procedure can be initiated by any of the following conditions: Failure of 120 volt single phase power to the turbomolecular pump controller, failure of 208 volt three 
phase power to the rotary pump, manual shutoff of the turbomolecular pump controller, overload of the turbomolecular pump, manual shutoff or overload of the rotary pump, intentional or inadvertent disconnection of any of the external wiring connected to the turbomolecular pump's logic interface. Automatic shutdown consists of the following steps: Isolation of the turbomolecular pump from the chamber and butterfly valve (whether open or closed) by the pneumatic gate valve located directly at the turbomolecular pump inlet, isolation of the turbomolecular pump exhaust from the rotary vane pump by the pneumatic inline valve, shutoff of power to the turbomolecular pump rotor, automatic venting of the rotor with dry nitrogen through the solenoid-actuated vent valve on the pump head. If line power is present, the rotary pump continues to operate, pumping against the closed inline valve.

Venting of the rotor stack in any turbomolecular pump upon shutdown is essential to prevent migration of adsorbed contaminants (chiefly hydrocarbons) from the rotors nearest the roughing pump upward to the rotors directly facing the ultra-high vacuum system. Since the pump head cannot be baked, such contamination would require that the pump be disassembled, cleaned and reassembled before it would cease to pose a contamination risk to the system.

Vent gas is clean, dry nitrogen taken from the same 80 PSI service that operates the pneumatic valves. A lowpressure regulator reduces its pressure to 1 PSI. It is used 
to vent both the turbomolecular pump and the chamber independently of one another.

Pumpdown of the chamber from atmosphere is accomplished through a bakeable metal-sealed angle valve mounted directly on the bell jar. An untrapped Edwards 12 cubic foot per minute two-stage direct-drive rotary pump charged with hydrocarbon fluid is temporarily attached directly to the valve by a several-foot length of rubber vacuum hose. The chamber is roughed by this means to a pressure of 0.5 to 1.0 torr, and then the valve is shut, the pump shut off and its intake immediately vented to air. The hose is removed along with the pump. Roughing in this fashion is more straightforward than the conventional procedure involving liquid nitrogen and sorption pumps. If it is not done correctly, though, it can contaminate the chamber with oil mist. The length of the vacuum hose as well as the strong convective flow of gas toward the pump over most of the pressure range covered is relied upon to keep oil mist backstreaming to negligible levels. Using this arrangement to rough below 0.5 torr, where gas viscosity becomes unacceptably low, is dangerous and is not advised. Pumping the chamber down from 0.5 torr with the turbomolecular pump by carefully throttling gas through the butterfly valve is a straightforward and rapid procedure. The foreline pressure should be observed and not allowed to rise above 200 millitorr, the approximate threshold for the "high-load" 
indication by the panel lamps on the turbomolecular pump controller. After the chamber pressure reaches about $10^{-6}$ torr (about one hour), the seat seal of the bakeable valve as well as any freshly installed conflat gaskets are helium leakchecked using the quadrupole mass spectrometer tuned to mass 4.

\subsubsection{Bakeout}

The chamber is baked after pumpdown in a rectangular tent of high-temperature fabric. The tent completely envelops the chamber except for the poppet valve actuator crank and the section of the turbomolecular pumping assembly up to the butterfly valve. These items project through appropriately located holes in the side of the tent.

The tent is constructed as a sandwich of ceramic wool insulation ( $1 / 2$ inch thick, 6 pounds per cubic foot density) between two layers of silicone rubber coated fiberglass fabric ( 7 ounces per square yard weight). The temperature rating of the latter material is the jower and is about $230 \mathrm{C}$. Blankets of similar construction insulate the aluminum baseplate during bakeout. The tent weighs about 40 pounds and can be stuffed into a box for compact storage. Velcro hook and loop material stitched to the outside of the tent allows flaps to be sealed when the tent is installed over the chamber.

The tent is supported by a wooden yoke attached to the steel monorail track overhead. It is hoisted from the wooden 
box in which it is stored by a block and tackle to the correct height to engage the end of the monorail track, a few feet to the side of the chamber. One wall of the tent is completely rolled up as a door, and the tent is hauled along the monorail until it covers the chamber. The tent is then closed by lowering the rolled side again and sealing it with velcro.

The volume of air inside the tent is heated by four 1000watt metal strip heaters supported vertically at the corners of the aluminum baseplate. Power to each is supplied by a dedicated 20-ampere variable autotransformer. The four variable autotransformers are housed in a cart. They are powered by a 3-phase 208 volt electrical service brought directly from the overhead bus. The temperature of the chamber is conveniently monitored as bakeout progresses by reading the chromel-alumel thermocouple attached to the sample. To reach a steady-state temperature of $150 \mathrm{C}$, the variable autotransformers are all set to a point on their scale marked "90". This corresponds to about 4000 watts of bakeout power. Bakeout is typically performed for about 24 hours from powermon to power-off. The chamber's interior then cools to room temperature over a period of six to eight hours, after which the tent is removed.

When the heaters are in operation, they incandesce with a dull cherry red color. No objects which may be damaged by such high temperatures should be allowed to touch them or come within a distance of a few inches of them. The stainless 
steel structure on which they are mounted acts as a radiation shield, protecting the inside lower corners of the tent from direct blackbody heating. An unlikely but not impossible accident could occur if the tent were to become partially or fully detached from its supporting structure during a bakeout. It would then rest unacceptably close to the strip heaters. The tent should therefore be inspected periodically for structural integrity. Particular attention should be given to the stitching where the walls attach to the top and to the four suspension points, which are small metal plates clamped through the top of the tent.

\subsubsection{Manipulator}

The sample manipulator is a Vacuum Generators HPLT 165 unit mounted upright. It provides precise translation of the sample along three orthogonal axes and also provides two rotational degrees of freedom. The primary rotation consists of motion of the sample holder about the vertical axis. The RD2S rotary drive integral to the HPLT 165 transmits this motion through a metal bellows-sealed wobble stick type mechanism. No dynamical seals exist in the ultra-high vacuum envelope. The secondary rotation occurs in the sample holder module at the bottom of the manipulator. It is driven by a micrometer-operated translation feedthrough which is coaxial with the RD2S wobble stick drive. A diagram of these rotational motions is given in Figure 12 . The translation 
Caption for Figure 12:

Schematic drawing of the vacuum chamber, sample holder and time-of-flight analysis port, with important axes shown by heavy arrows. Other ports are omitted for clarity. Axes 1, 2 and 3 are mutually orthogonal when two-photon photoemission data are being collected. Axis 1 is the primary rotation axis of the manipulator. Axis 2, the secondary rotation axis, lies in the plane of he sample surface. The sample is rotated about it to collect dispersion data. Axis 3 is the axis of the angle-resolved time-of-flight detector and is also the centerline of the port projecting to the left, in which the detector is installed. The perpendicular distance between 1 and 2 is 3.50 inches. 


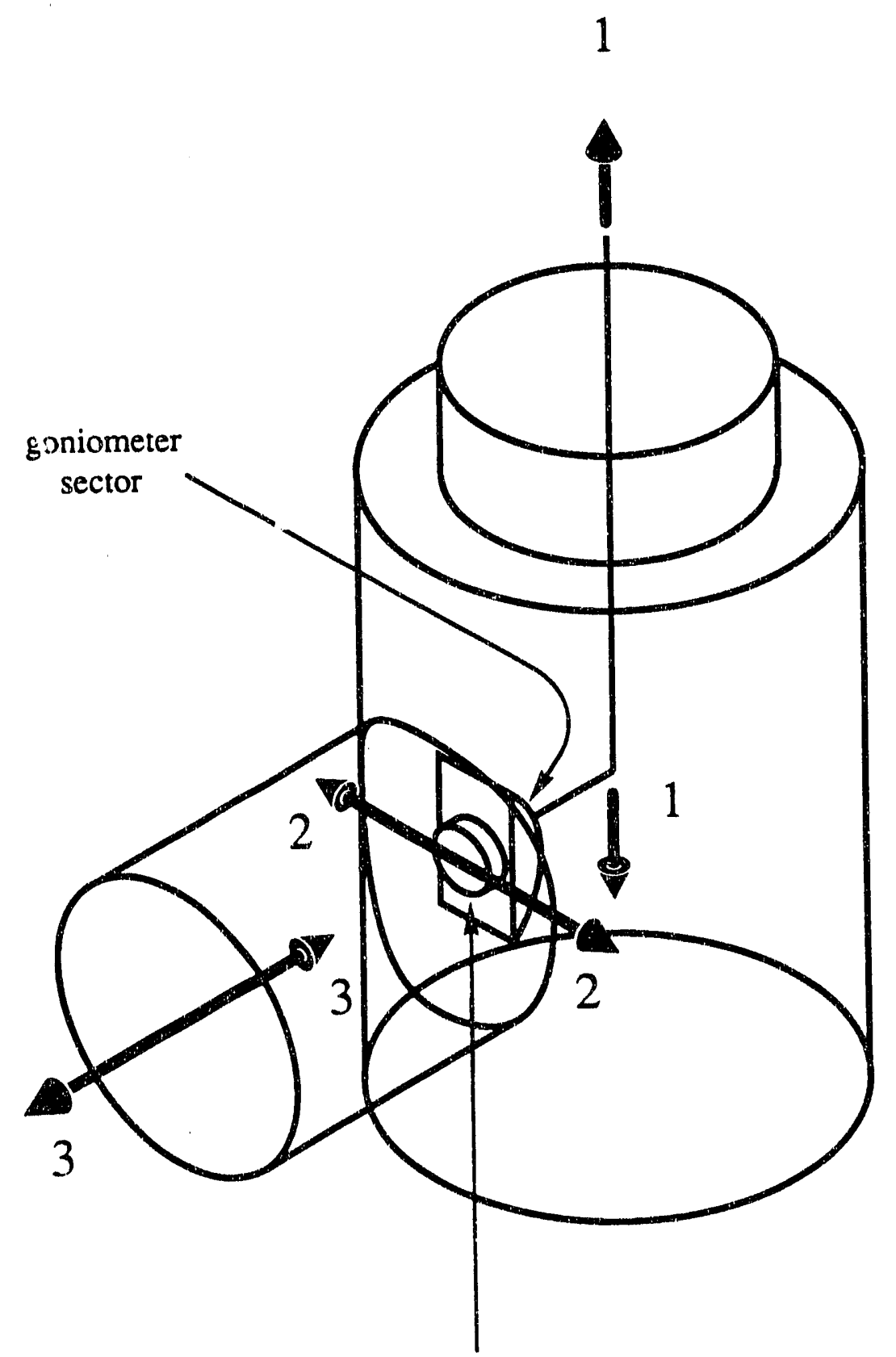

sample

Figure 12 
feedthrough is also sealed by a metal bellows.

Three custom features are present in this manipulator. Four unconnected electrical feedthroughs were installed for connection of Faraday cups and auxiliary or dummy samples which may need to be supported by the manipulator. A tefion outrigger bearing steadies the vertical hotion platform of the manipulator inside the ultra-high vacuum region. Without this, the rample would effectively reside at the ond of a long cantilever and would thus be more susceptible to mechanical vibrations driven by the turbomolecular pump. The offset between the centerpoint of the sample surface and the vertical axis of primary rotation was designed as 3.50 inches rather than 2.50 inches which is the standard dimension. The sample thus swings about a 7.00 inch diameter circle oriented horizontally in the chamber. Clearances between such bulky instruments as the LEED optics and the time-of-flight tube are more relaxed than they would be if this dimension were 5.00 inches. A particular advantage of this axrangement is that it renders installation of the manipulator in the presence of the other instruments much less perilous.

\section{1 .5 Sample Holder}

The sample holder itself comprises the lower end of the manipulator and contains the secondary rotation mechanism, which is used in angle-resolved two-photon photoemission experiments. The requirements placed upon the sample holder 
are particularly demanding. It must allow the sample to be mobile but must support it rigidly. Moving parts must be assembled with close tolerances for precise motion, but must be prevented from the galling and seizing to which such mechanisms are prone in ultra-high vacuum. The sample holder must be robust enough to resist the twisting and bending forces transmitted to it by the copper ropes attached near the sample. It must include provisions for sample annealing to several hundred degrees celsius and also be compatible with sample cooling below liquid nitrogen temperatures. In order to avoid excessive heat load on the sample cooling device, and also to avoid uncontrolled baking of the angular motion mechanism upon sample annealing, a highly efficient but mechanically rigid thermal breakpoint must be positioned between the sample and the rest of the sample holder assembly. The sample temperature must be measured accurately by a very compact sensor that is compatible with ultra-high vacuum and also usable over the full temperature range experienced by the sample. In general, metal parts must be made of nonferromagnetic materials. Insulating parts surin as ceramics and vacuum-compatible plastics must be prevented from accumulating electrostatic charges, which can introduce dramatic artifacts in angle-resolved photoelectron spectra. Finally, the angle of the sample normal relative to the detector axis must be able to be read in a simple, reliable way. 
The axis of secondary rotation is horizontal. The laser beam illuminates the sample surface through a quartz viewport located beside the time-of-flight detector, and the plane of reflection moves as the secondary rotation axis of the sample is varied. The angle of incidence of the laser beam relative to the sample normal is $60^{\circ}$ when the sample normal is horizontal, and it increases to $62^{\circ}$ when the secondary rotation angle is set to its full travel, $20^{\circ}$ up or down. When the sample normal is nearly horizontal, laser light reflected from the sample exits a quartz window. As the sample normal is rotated up or down relative to the detector axis, reflected laser light strikes the inside of the magnetic shields surrounding the flight region. This surface is coated with colloidal graphite. Laser light striking or scatterring from this surface has not caused any problems in the experiment.

Due to the symmetry properties of the wave funtions of image potential states, photoemission from them is most efficient when the normal component of the electric vector of the photoemitting light is maximized. ${ }^{38}$ When photoelectron spectra are collected at different secondary rotation angles of the sample, this component changes, and the signal level of the two-photon photoemission experiment is expected to scale with it. The desire to minimize this effect was the reason the experiment was designed with as small an angle between the laser beam and the secondary rotation axis as possible. With 
the laser polarization horizontal, the normal component of the electric field vector changes by about $6 \%$ when the secondary rotation angle is changed from $-20^{\circ}$ to $+20^{\circ}$. The magnitude of this effect would be larger if the secondary rotation axis were, for example, vertical. Then the change in the normal component of the electric vector would be about $35 \%$ upon rotation from $-20^{\circ}$ to $+20^{\circ}$.

Two different sample holders were used in the new vacuum chamber. The earlier version was the modified Vacuum Generators RSM2T unit originally supplied with the manipulator. The secondary rotation was driven by the translation feedthrough in the RD2S rotary drive using a seesaw mechanism with a $1: 1$ drive ratio. It could be driven approximately nine degrees up and nine degrees down from the middle of its travel.

The sample was clamped in the standard Vacuum Generators VZHSTSK heater assembly. This heater is a module containing a tungsten hairpin folded repeatedly back upon itself. It is resistively heated by application of direct current. Because of the small area enclosed by the hairpin, its inductance is kept to a minimum. The filament is threaded through thin alumina tubes which insulate it electrically from the small molybdenum box which comprises the exoskeleton of the heater cartridge. The front of this molybdenum box supports the sample. Heat is transferred from the ceramic tubes to the molybdenum box by both conduction and radiation. An appendage 
on the front of the molybdenum box is provided for attachment of a metal cooling braid. Four sapphire balls captured behind the molybdenum box act as thermal and electrical breakpoints, minimizing heat flow between the mounting plate which anchors the heater to the body of the sample holder and the molybdenum box. While the sapphire ball arrangement appears to offer a larger effective contact area for conductive heat transfer than might be desired, it is adequately rigid and quite resistant to damage from mechanical shock and overloading. The polar angle of the sample was read from the barrel of the micrometer on the outside of the chamber. The secondary motion mechanism of this sample holder immediately proved to be inaccurate, imprecise, and mechanically unsound. Unpredictable jumps in the sample's rotation angle by about $2^{\circ}$ and lateral jumps in its position relative to the laser and time-of-flight analyzer made reliable angle-resolved photoemission data extremely difficult to collect. In addition, the limited angular range of the device required that the sample be ground as a wedge in order to collect data from the center of the surface Brillouin zone to about 0.15 $\AA^{-1}$. An improved sample holder was designed.

The newer sample holder is designed to allow total secondary rotation of $40^{\circ}$. It is driven by the RSM2T translation feedthrough using a seesaw mechanism similar to the earlier sample holder. Diagrams of this device appear in Figure 13 and Figure 14. A $2: 1$ reduction of the force 
Caption for Figure 13:

Right-hand side view of the specially designed sample manipulator for angle-resolved two-photon photoemission experiments. The electrical wires supplying power to the heater as well as the appendage for cooling the sample are omitted for the sake of simplicity. The thermal breakpoints are contained within the heater module. The scale measures angles from $-20^{\circ}$ to $+20^{\circ}$. While dimensions taken from this drawing should not be used for design purposes, it is practically the actual size of the object. 


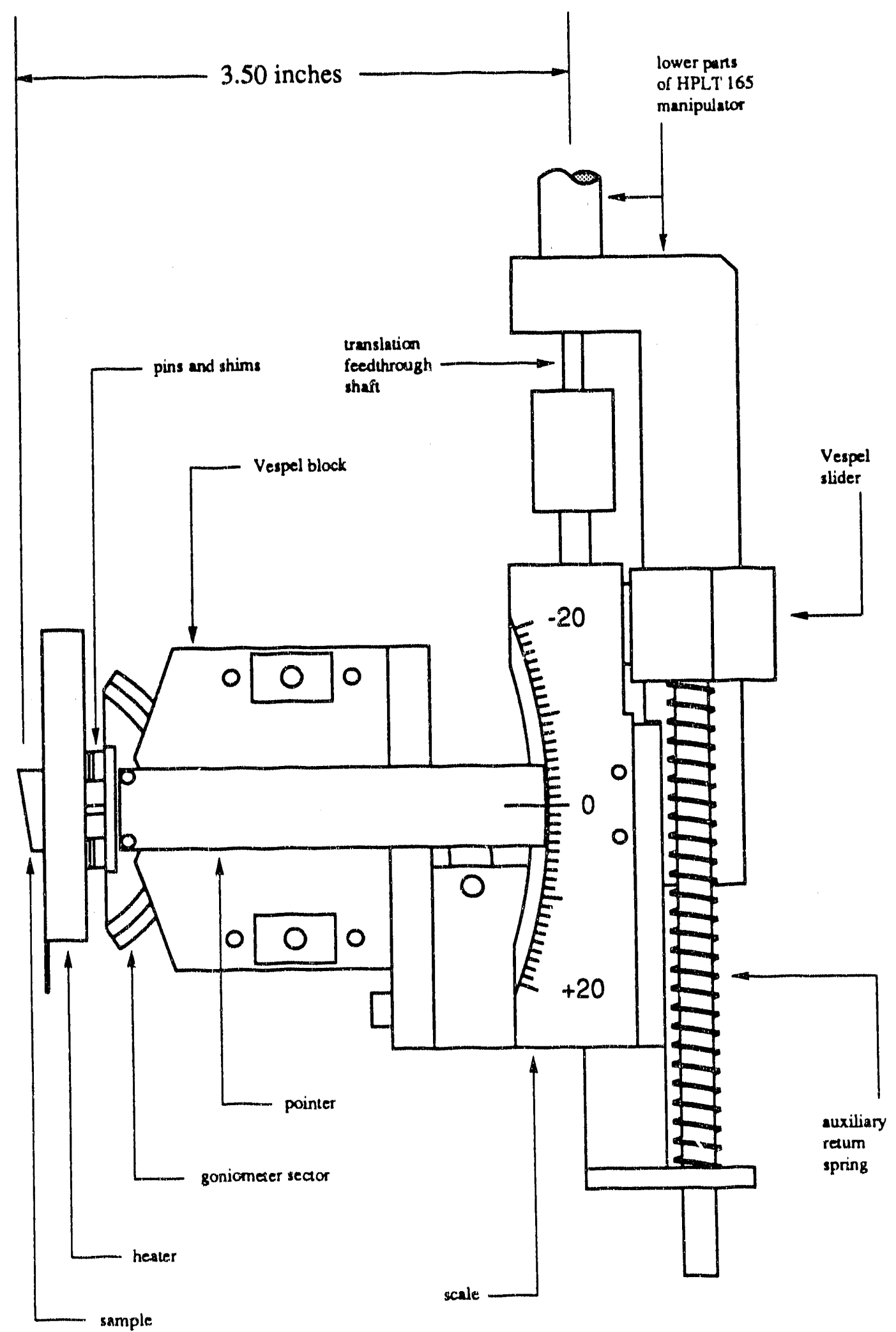

Figure 13 
Caption for Figure 14:

Drawing of the sample holder partially disassembled to illustrate the action of the secondary rotation mechanism. The edge of the goniometer sector is a lip, which engages a track machined into the Vespel block. In (a), the mechanism is at the $-20^{\circ}$ position. In (b), it is at $0^{\circ}$, and in (c), it is at $+20^{\circ}$. The auxiliary return spring, cooling appendage and wiring are all omitted for clarity. This drawing is approximately $1 / 2$ scale. Dimensions taken from it should not be used for design purposes. 


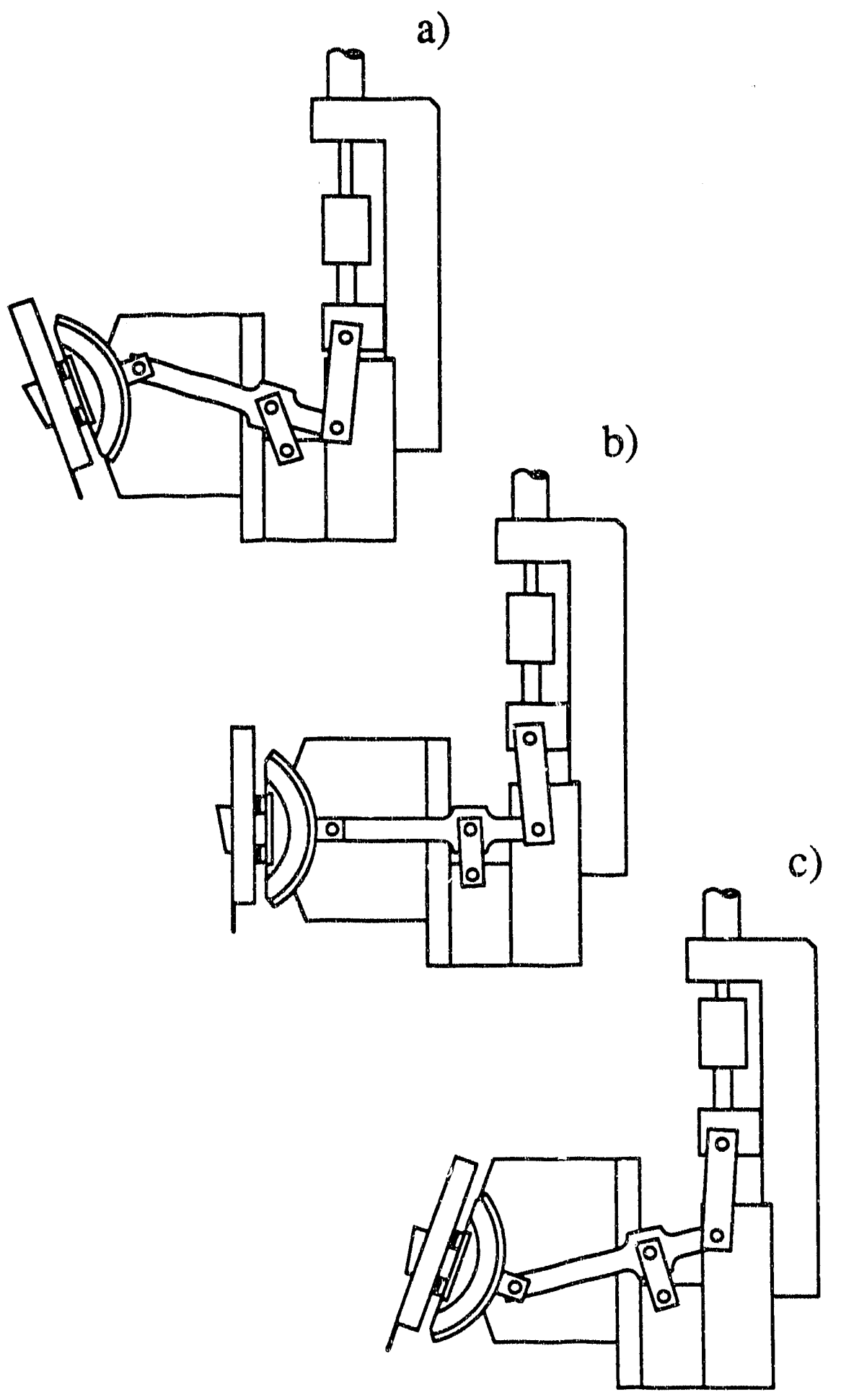

Figure 14 
transmitted from the translation feedthrough allows the greater angular range to be achieved. Most of the parts were machined from type 304 stainless steel. Beryllium copper and silicon bronze were used for certain moving parts where extra insurance against galling and seizing was needed. Aluminum was used as a structural material, for parts where toughness was not critical. Where alignment between mating parts was essential, 0.094 inch stainless teel dowel pins were used. Pin holes were honed to a light press-fit clearance at one end of the pin and a slip-fit clearance at the other. Blind holes and trapped volumes were vented by a number 60 wire size drill. To minimize galling of the stainless steel parts against one another, they were lightly gold plated wherever this was possible without altering p.ess-fit clearances. Parts that could not be plated were lightly electropolished. Immediately before assembly, a paste of molybdenum disulfide powder in ethanol was liberally applied to both sides of all running bearing surfaces as a lubricant. The excess dried powder was blown off after assembly.

The critical secondary motion mechanism was built as a goniometer. A precisely turned stainless steel sector is captured at both sides by tracks machined into blocks of Vespel sP-3. This is a molybdenum disulfide-filled polyimide plastic which is advertised as meeting the outgassing requirements of ultra-high vacuum applications. It is intended to be used in low-friction moving parts in vacuum 
environments. Its structural integrity is supposed to remain intact to a temperature of at least $250 \mathrm{c}$. The use of plastic was deemed essential in this case since a close running fit was needed between the sector and the tracks, but absolutely no galling could be tolerated. The final clearance was established by lightly honing the surfaces of the tracks with 600 grit silicon carbide paper. Exterior surfaces of the Vespel blocks were coated with vapor-deposited nichrome alloy to a thickness of about 100 ohms per square. This was essential to provide grounding of the insulating plastic surfaces in order to drain off the surface charge likely to accumulate in vacuum. Extensive cleaning of the Vespel pieces prior to installation appeared to efficiently remove the molybdenum disulfide filler from the surfaces. This was a disadvantage, and probably resulted in the filled vespel exhibiting no better frictional properties than the unfilled material. The tracks supporting the goniometer sector needed to be lubricated before assembly along with the other moving parts.

The goniometer places the secondary rotation axis in the region of space in front of the sample holder. The crystal needs to be carefully positioned in its mount to place this axis very close to its surface plane. To address this requirement, four beryllium copper pins and a set of 0.020 inch thick washer-like shims were machined to support the heater cartridge, align it, and allow its distance from the 
goniometer sector to be increased or decreased in intervals of 0.020 inches. For sample thicknesses between 0 and 0.250 inches, this arrangement is intended to provide surficient adjustability that the secondary rotation axis need not lie more than 0.010 inches from the plane of the sample surface. This tolerance corresponds to $0.25 \%$ of the photoelectron flight distance.

The translational motion feedthrough shaft operating the goniometer mechanism provides positive, screw-driven displacement in the downward direction but relies upon an internal return spring for upward displacement. The force available in the upward direction is thus only about four pounds. This is less than is required to operate the goniometer. An auxiliary return spring therefore had to be built. A Vespel slider engages a Vespel pawl attached to the translation feedthrough shaft and is driven by two stainless steel springs with approximately ten pounds of force in the upward direction. The angle of the goniometer sector can be read accurately by an attached pointer which moves along a stationary engraved scale. The uncertainty in reading this angle is $0.2^{\circ}$. Angles to $20^{\circ}$ above and below the midpoint of travel may be read.

Whereas the base pressure of the chamber before introduction of the new sample holder was $1 \times 10^{-10}$ torr, the base pressure afterward was about $3 \times 10^{-9}$ torr. This base pressure was observed to drop by a factor of four over a 
several-week timescale. The increased base pressure is tentatively attributed to outgassing from the Vespel pieces, whose vacuum compatibility may not be as perfect as advertised.

The sample temperature was monitored by a chromel-alumel thermocouple, which was chosen from the large variety of temperature sen: irs available for several reasons. It is compact, easily placed in intimate contact with the silver crystal, and fully compatible with high temperature and ultrahigh vacuum. It is less sensitive at cryogenic temperatures than diodes or resistance thermometers, but these are bulkier and are often vulnerable to damage at temperatures above about $150 \mathrm{c}$. The chromel-alumel junction was made from 0.003 inch diameter bare wires from omega and was twisted tightly between cardboard-padded surgical clamps. It was clamped against the sample by the molybdenum spring clips which attached the sample to the heater assembly. The chromel and alumel leads were about two inches long and were clamped at the other end against chromel and alumel lugs inside of a small, specially made Macor terminal block. These lugs were attached to the chromel and alumel thermocouple feedthroughs provided in the HPLT 165 manipulator by spotwelded chromel and alumel wires of 0.010 inch diameter. The temperature of the sample was monitored and the sample heater was controlled by a Eurotherm $818 \mathrm{P}$ controller to which a Kepco KS36-15 programmable power supply was slaved. 
In order to test the performance of the thermal breakpoints in the sample heater assembly, a second chromelalumel junction was spotwelded directly to the goniometer sector and connected to a second pair of feedthroughs. With the sample at a steady-state temperature of $400 \mathrm{C}$, the goniometer sector reached $160 \mathrm{c}$. Thus the thermal breakpoints were far from perfect. If the sample were annealed at about $500 \mathrm{C}$, thermal damage to the Vespel blocks supporting the goniometer sector would be a significant risk.

\subsubsection{Sample cooling}

Sample cooling was provided by a liquid helium flow cryostat connected to the sample heater module by flexible copper ropes. This assembly is shown in Figure 15. When the HPLT 165 manipulator was originally ordered, provisions were included for sample cooling by a flow of liquid nitrogen through a heat exchanger near the sample. This heat exchanger was connected to the sample heater by a single copper rope about two inches long. Using this liquid nitrogen cooling arrangement, the sample reached a temperature of about $120 \mathrm{~K}$. This was not. low enough to adsorb xenon. For this reason, the liquid nitrogen cooling was disconnected and liquid helium cooling was adopted instead.

The helium flow cryostat is an Air Products LT-3B-110 unit recycled from the Gallo chamber. It was mounted rigidly in the chamber below the main instrumentation level. Heat is 
Caption for Figure 15:

Diagram of the sample holder with appendage for cooling the sample attached. The copper braid which provides heat sinking is flexible enough to allow primary rotation sf $\pm 180^{\circ}$ from the midpoint of travel. Performance of the system is such that, when the temperature at the lower end of the braid is $2.5 \mathrm{~K}$, the temperature of the sample is $85 \mathrm{~K}$. This drawing is approximately $3 / 10$ full scale. Dimensions taken from it should not be used for cesigr purposes. 


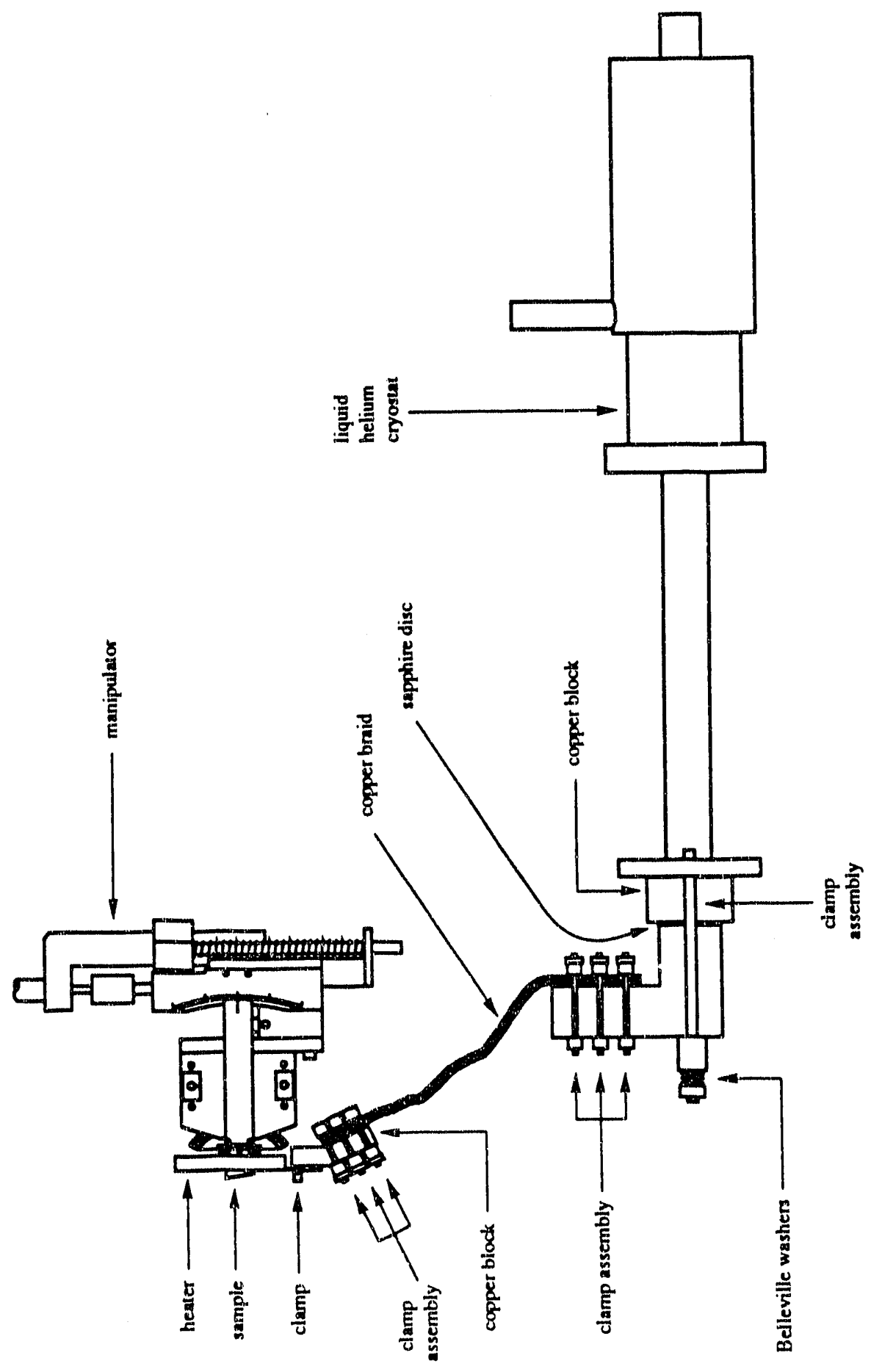

Figure 15 
removed from the sample through three OFHC copper braids, each of which has an effective cross-sectional area of $0.068 \mathrm{~cm}^{2}$, as determined using the braid's weight per unit length and the known density of copper. The upper end of the braid terminates in a solid copper block designed to attach to the sample heater. The braid was tightly clamped to this block by a set of three stainless steel screw clamps. The assembly of the block, braid and clamps was pre-loaded by tightly squeezing it in an arbor press while the screws were brought up to their final torque. This procedure was intended to increase the contact area at the braid-block junction. A similar assembly existed at the bottom of the braid. The copper block at this end was clamped against the end of the cryostat by a stainless steel screw clamp which incorporates stacks of compressed stainless steel Belleville washers to prevent loosening of the assembly due to thermal contraction of the parts upon cooling to cryogenic temperatures. Between the copper block and the end of the cryostat, a disc of polished sapphire acts as a thermally conducting electrical insulator. Both metal surfaces bearing upon the sapphire disc are flat and polished to a mirror finish to ensure optimal contact area.

The copper block attached to the lower end of the braid contains a silicon diode temperature sensor whose leads are connected to an electrical feedthrough on the 6.00 inch $0 . D$. flange supporting the cryostat. This diode provides a 
convenient, accurate temperature readout, but it is not able to experience temperatures over $150 \mathrm{C}$. When the sample was cooled to a steady-state temperature of $65 \mathrm{~K}$, the diode read $25 \mathrm{~K}$. Thus the braid supported a $40 \mathrm{~K}$ temperature differential from top to bottom. Most of this temperature differential is believed to be due to heat leaking through the thermal breakpoint in the Vacuum Generators VZHSTSK sample heater module.

\subsection{Sample Preparation}

The sample used in this experiment was a disc of single crystal silver of 99.9998 puxity. It was obtained from Monocrystals, Incorporated. Its face was oriented parallel to the (111) crystal plane. Its dimensions as it was received from the manufacturer were $1 / 2$ inch diameter by $3 / 16$ inch thick. For use in angle-resolved experiments, it was ground to a $9.5^{\circ}$ wedge, with its gradient aligned along the $\bar{\Gamma}-\bar{K}$ direction of the surface Brillouin zone.

orientation of the crystal was done using the technique of laue backscatterring on the community-use instrument in the Chemical Sciences Division at Lawrence Berkeley Laboratory. Diffraction data was collected using the computer-interfaced position sensitive detector manufactured by Multiwire Laboratories. This instrument uses the proprietary Northstar software package. The $x$-ray tube, containing a copper target, was operated at 20 kilovolts and 20 milliamperes. A 1 
millimeter collimator was installed. The distance from the collimator tip to the sample surface was 50 millimeters.

To align the crystal's azimuthal angle accurately with $\bar{\Gamma}-\bar{K}$, the projections of the principal crystal axes in the surface plane were determined. This was accomplished by the following procedure: An accurately aligned (111) diffraction pattern was obtained. The polar angle of the crystal was then rotated $54.7^{\circ}$, which is the angle between the (111) lattice vector and any one of the vectors (100), (010) and (001). with the polar angle thus fixed, the azimuthal angle was slowly scanned with the motorized goniometer. The Laue diffraction pattern was observed in real time, with the display set to medium persistence, until a well-oriented (100) diffraction pattern was apparent. This pattern was easily recovered upon $120^{\circ}$ rotations of the azimuthal angle, as would be expected. In this manner, the orientations of the crystal axes projected into the (111) face were determined and were marked on the crystal with a felt tip pen.

After the polar angle of the sample was returned to its original setting, the marked crystal axis directions could be matched up unambiguously with particular high-symmetry lines in the (111) diffraction pattern. This alignment is diagrammed in Figure 16. The cross-hair option was engaged in the data display window, and final alignment of both the polar and azimuthal crystal rotations was ascertained with the help of a $\mathrm{C}_{6}$-scribed transparent plastic screen overlay. Alignment 
Caption for Figure 16:

Diagram of Laue pattern from silver (111) with orientation of projection of crystal axes labelled. Heavy arrows indicate orientation of two of the six $\bar{\Gamma}-\bar{K}$ lines in the surface Brillouin zone. Dotted circles are intended as guides to the eye and are not present in the actual Laue pattern. 


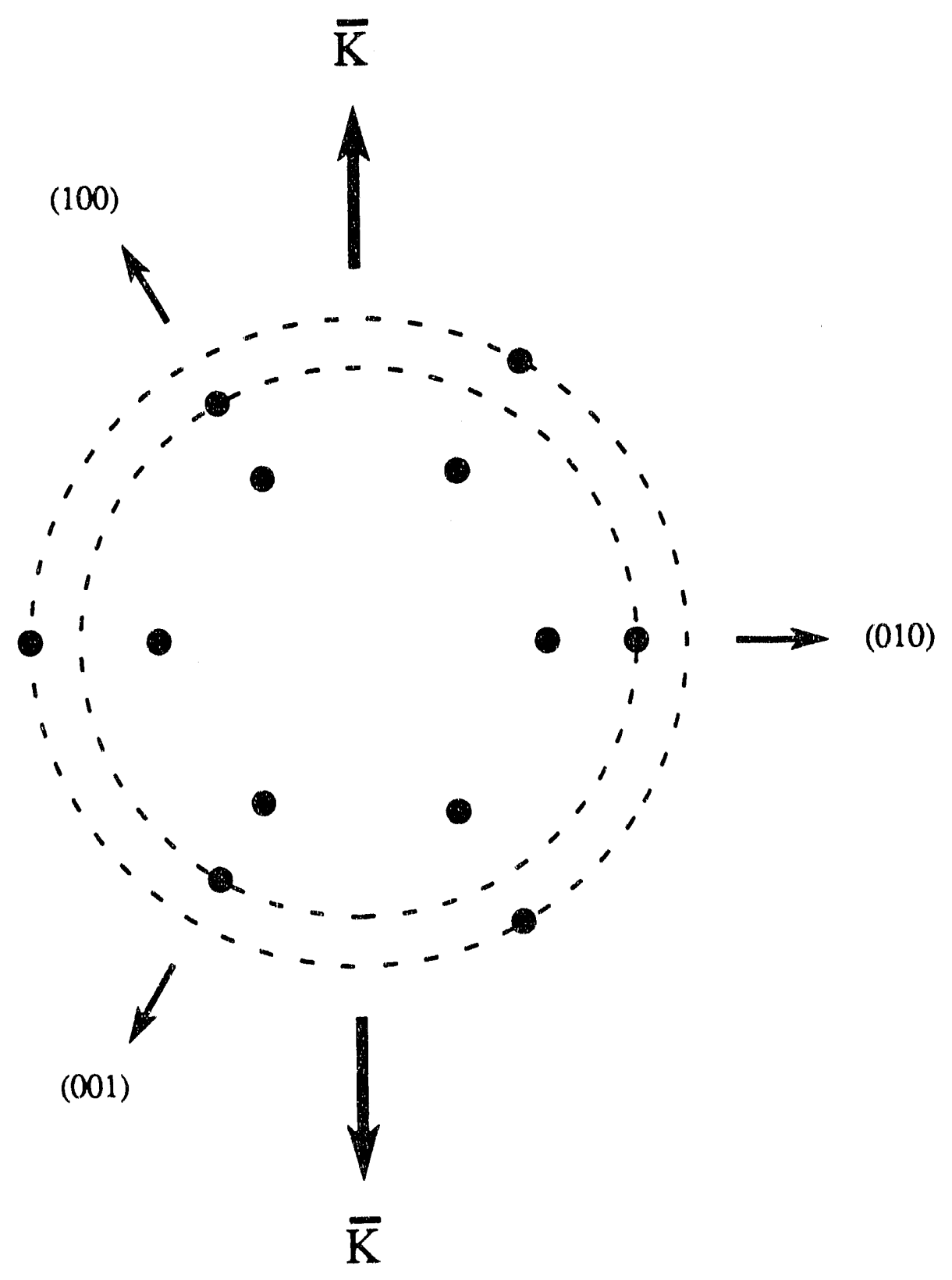

Figure 16 
uncertainties were, for the polar angle, $\pm 0.5^{\circ}$, and for the azimuthal angle, $\pm 2^{\circ}$. As can be deduced readily by geometrical considerations, the six $\bar{\Gamma}-\bar{K}$ directions in the surface Brillouin zone 1 ie $30^{\circ}$ to either side of the projected crystal axes in the (111) plane. ${ }^{21}$ The crystal was removed from the motorized goniometer for grinding, described below. To enable the (111) surface to be rotated between $0^{\circ}$ and $20^{\circ}$ relative to the time-of-flight detector axis using the Vacuum Generators RSM2'T sample holder, the back of the crystal was ground as a wedge. To this end, a specially constructed grinding jig was used. It consisted of a hollow cylindrical body with a circular stainless steel foot. This foot defined a reference plane for grinding on a flat, abrasive-covered plate. Inside the cylindrical body, a spring-loaded sample holder was held by setscrews so that its orientation could be adjusted. The sample was attached to an accurately machined plunger by sticky wax and the plunger was held in a socket and driven along the axis of the sample nolder assembly by a spring. In this way, the crystal was pressed into contact with the abrasive-covered plate when the foot of the grinding jig rested upon the plate.

The body of the grinding jig, supporting the sample and sample holder in its center, was rigidly clamped in the Laue camera in accurate alignment with the $x$-ray beam. The sample nolder assembly was then adjusted with the setscrews relative to the stationary body of the jig until an accurately centered 
(111) diffraction pattern was obtained. The $\bar{\Gamma}-\bar{K}$ direction was made vertical by rotation of the cylindrical body of the $j i g$. At this point, the stainless steel foot defined a plane parallel to the (111) plane within the $0.5^{\circ}$ uncertainty of the polar angle measurement.

A machined stainless steel ring with a $9.5^{\circ}$ wedge angle between its top and bottom surfaces was clamped onto the foot. The gradient of the wedge-shaped ring was marked by narrow notches machined into it. The wedge-shaped ring was rotated relative to the axis of the jig until its gradient was in a vertical plane and was thus aligned with $\bar{\Gamma}-\bar{K}$. The uncertainty in this procedure was $2^{\circ}$ of azimuthal angle. The ring was then clamped solidly to the jig and provided a reference surface for the grinding of the $9.5^{\circ}$ wedge on the back of the crystal.

Gross removal of material was accomplished with $200 \mathrm{grit}$ silicon carbide abrasive paper, liberally flooded with water. The abrasive paper had an adhesive backing and was supported on a sheet of plate glass. Once the wedge had been established, scratches were removed with successive application of $400 \mathrm{grit}$ and the $600 \mathrm{grit}$ silicon carbide paper in the presence of flowing water. Finally, 6 micron diamond paste with a mineral oil carrier was used on a rotating wheel covered with nylon fabric to bring the back of the crystal to the desired smoothness.

After grinding of the wedge, the sample was clamped in an 
aluminum carrier, and notches were cut into its sides for mounting in the Vacuum Generators VZHSTSK sample holder. Two notches were cut on oppposite sides of the crystal and were oriented to be at opposite ends of the wedge gradient. They were cut by electrical discharge machining, using an electrode made from 0.040 inch thick tungsten sheet.

once the wedge angle and notches were completed, The (111) face of the crystal was polished. It was mounted in Koldmount (produced by Buehler, Limited) on a sheet of plate glass previously sprayed with release agent. The plasticencased sample that resulted was polished in several steps. First, it was made flat by grinding on 600 grit silicon carbide paper stuck to a plate glass slab. Water was flowed over the abrasive paper. Second, the sample was ground on a rotating wheel covered by nylon fabric upon which 6 micron diamond paste had been spread. Mineral oil was used as an extender. Third, 1 micron diamond paste was used on a rotating wheel covered by Leco Imperial Cloth. Ultrasonic cleaning was performed between steps to minimize transferral of abrasive particles. The sample was then released from the Koldmount block. Finally, just before installation in the ultra-high vacuum chamber, the sample was chemically polished with a solution composed as folows: 4.5 grams of potassium dichromate, 0.7 grams sodium chloride, 10 milliliters concentrated sulfuric acid and 214 milliliters of water. This formula was used earlier in surface studies of silver, ${ }^{123}$ 
but, unfortunately, it has not been possible to find references to it in any published work to date. Very brief etches using small doses of this solution followed rapidly by washes with deionized water gave the required polishing action. The sample was washed with an ethanol solvent. Acetone was found to leave a film upon evaporation.

After installation in the ultra-high vacuum system, the sample was cleaned by sputterring with $500 \mathrm{eV}$ argon ions at $5 \times 10^{-5}$ torr argon pressure (uncorrected for ion gauge response) and 20 milliampere filament current. This procedure lasted 20 minutes, during which the sample temperature was held at around 200 c. After annealing for approximately one hour at $425 \mathrm{C}$, retarding field Auger analysis showed no evidence of surface contamination. In particular, the silver-carbon peak ratio attained its canonical value for clean silver. ${ }^{124}$ The typical daily sample cleaning procedure consisted of two steps. First, the crystal was sputterred with $500 \mathrm{eV}$ argon ions at a pressure of $5 \times 10^{-5}$ torr (uncorrected) and 20 milliampere filament current for 20 minutes at $200 \mathrm{C}$. Beam current intercepted at the sample was about 10 microamperes. Then the sample was annealed in ultra-high vacuum for 20 minutes at $425 \mathrm{c}$. Surface order and absence of contamination were maintained by this procedure.

\subsection{Laser System}

The laser used in this experiment was a coherent 702-2 
synchronously pumped dye laser. Typically, it was operated with the saturable absorber jet turned off. It was cavity dumped at a repetition rate of either 0.95 or $1.90 \mathrm{MHz}$. Average power was about 100 milliwatts. Pulse durations were about 10 ps as measured by autocorrelation. Pulse energies were about $10^{-7}$ joules. Over the wavelength range of 550 to $620 \mathrm{~nm}$ (photon energies 2.25 to $1.99 \mathrm{eV}$ ), Rhodamine $6 \mathrm{G}$ in ethylene glycol served as the gain medium. From 610 to $660 \mathrm{~nm}$ (photon energies 2.03 to $1.88 \mathrm{eV}$ ), DCM in a proplylene cartenate/ethylene glycol mixture was used as the gain medium. The dye laser was pumped by the second harmonic $(527 \mathrm{~nm})$ output of a coherent Antares modelocked CW YLF laser. This laser operated at a repetition rate of $76 \mathrm{MHz}$ and produced pulses of 80 ps duration in the green, as measured by a fast photodiode and sampling oscilloscope. The dye laser was pumped with an average power of about 1.5 watts.

Excitation of the image potential state from states below the Fermi level required near-UV photons, which were produced as the second harmonic of the dye laser output. UV wavelengths between 330 and $315 \mathrm{~nm}$ ( 3.76 to $3.94 \mathrm{eV}$ photons) were covered by frequency doubling in an angle-tuned lithium iodate crystal of $5 \mathrm{~mm}$ length. UV wavelengths between 315 and $275 \mathrm{~nm}$ ( 3.94 to $4.50 \mathrm{eV}$ photons) were covered by an angletuned $1 \mathrm{~cm}$ KDP crystal. Doubled power was $3 \%$ to $5 \%$ of the fundamental when KDP was used and $8 \%$ to $12 \%$ of the fundamental when lithium iodate was used. In both cases, the beam of 
about $1 \mathrm{~mm}$ diameter was focussed into the crystal by a $5 \mathrm{~cm}$ focal length plano-convex lens. The output beam was recollimated by a $20 \mathrm{~cm}$ foral length lens. The UV and visible wavelengths were split by a dichroic mirror for independent adjustment of their polarizations and their collimation. Their pathlengths were also balanced to within about $2 \mathrm{~mm}$. They were then overlapped and recollimated by another dichroic mirror and focussed onto the sample. The spot size at the sample was $0.5 \mathrm{~mm}^{2}$. When the two wavelengths had to be handled by the same mirror, uncoated aluminum mirrors (Newport $A L-2$ ) were used in order to provide an acceptable compromise between UV and visible reflectance. The final lateral and temporal overlap of the two wavelengths could be optimized by observing the count rate due to two-photon photoelectrons. Total count rates were typically between 1000 and several thousand per second. As much as 958 of the total count rate was attributable to two-photon photoemission from the image potential state. The remainder was due to single-photon photoemission.

space charge broadening of the photoelectron energy distribution has been described in the two-photon photoemission literature. ${ }^{65,40}$ When image potential states were observed, the threshold for unacceptably strong space charge broadening was reported to occur at about $5 \times 10^{4} \mathrm{~W} / \mathrm{cm}^{2}$ peak intensity. In the present work, visible (probe) pulses incident on the sample carried about $10^{-8}$ joules of energy in 
about $10^{-11}$ seconds, deposited in an area of about $5 \times 10^{-3} \mathrm{~cm}^{2}$. Thus the peak intensity was $2 \times 10^{5} \mathrm{w} / \mathrm{cm}^{2}$. Features in the photoelectron kinetic energy distribution attributable to photoemission from the image potential states still exhibited widths of about $50 \mathrm{meV}$ at half-maximum height. It is likely that space charge broadening of the photoemission features depends somewhat on laser pulse energy, and not merely upon the peak intensity as reported in the literature. In the present experiment, which uses pulses of very low energy, space charge broadening was negligible even when the full, unattenuated power of the laser beam was used to excite the sample.

\subsection{Time-of-Flight Analysis}

"lectron energy analysis by the time-of-flight method was of fundamental importance in this experiment. In this section, the relevant experimental literature will be reviewed briefly. The detector used in this experiment will be described. The issues of detector collection angle, energy resolution and conversion of flight times to kinetic energies will be addressed. A significant complication in time-offlight energy analysis of photoelectrons from solid samples is the contact potential difference between the sample and the detector. The approach to this problem which was adopted in the present work will be explained. Finaliy, the time-offlight method will be compared with the alternative, the 
electrostatic deflection analyzer.

\subsubsection{Literature}

An early application of time-of-flight energy analysis to photoelectron spectroscopy was by Tsai, Baer and Horovitz. ${ }^{125}$ They used continuous-wave vacuum ultraviolet illumination to ionize methyl iodide in the gas phase. A pulsed collecting field wh - applied at a frequency of $3.3 \mathrm{MHz}$ to the collection region to inject short bursts of photoelectrons into a $3 \mathrm{~cm}$ long magneticalily shielded flight tube. The effective solid angle of collection was 0.02 steradian. The flight tube was gold plated in an attempt to create a region free of fields due to contact potential differences between patches of dissimilar work function on the surface. A channel electron multiplier detected the electrons and timing was determined with a time-to-amplitude converter. As is required when such electronics are used, the photoelectrons were collected in the pulse-couriting regime. Using the delayed coincidence technique in a range of kinetic energies between 0 and $1 \mathrm{eV}$, the authors reported $50 \mathrm{meV}$ resolution and count rates of around $1000 / \mathrm{s}$.

Bachrach, Brown and Hagströrn ${ }^{126}$ built an angle-resolved photoelectron spectrometer for use with solid samples and synchrotron radiation at $100 \mathrm{eV}$ photon energy. Core and valence electronic levels of $\mathrm{Si}(111)$ and $\mathrm{NbSe}_{2}$ were probed. synchrotron pulses of $1.5 \mathrm{~ns}$ duration and a repetition rate of 
1.3 MHz excited the sample. Photoelectrons initially encountered a retarding field, intended to improve energy resolution, and then traversed a six inch long magnetically shielded flight region. They were detected by a chevron microchannelplate assembly, and flight times were analyzed by the delayed coincidence technique using a time-to-ampsitude converter. The angular resolution was $\pm 1.0^{\circ}$. The angle of the detector axis relative to the sample was varied by swinging the detector around the stationary sample. The quoted resolution at $5 \mathrm{eV}$ kinetic energy was $\pm 100 \mathrm{mev}$. No value was reported for a typical countrate obtained with this apparatus.

White and coworkers ${ }^{127}$ built a time-of-flight spectrometer for photoemission studies of gases using synchrotron radiation. These researchers used the same synchrotron source as Bachrach and coworkers, although the photon energy in the range of interest was 4 to $36 \mathrm{eV}$. The flight path was $28.5 \mathrm{~cm}$ long, was magnetically shielded, and was coated internally with colloidal graphite to reduce secondary photoemission due to collisions of photoelectrons with the walls. The angular resolution was $\pm 3^{\circ}$. A chevron microchannelplate with a $50 \mathrm{ohm}$ impedence-matched anode and a time-to-amplitude converter served as the detector and flight tirne analyzer. The construction of the microchannelplate assembly is treated in greater detail than in other articles. Also, the dependence of energy resolution upon uncertainty in 
timing and uncertainty in the electron's flight path is explained thoroughly. An energy resolution of \pm 58 was obtained over the energy range studied. No information was given on count rates.

An instrument built by Bokor for two-photon photoemission used time-of-flight energy analysis. ${ }^{101,100}$ The sample in this experiment was the (110) face of the semiconductor InP. The probe photon energy was $10.5 \mathrm{eV}$. Photoelectrons with energies around $6.5 \mathrm{eV}$ were detected with resolution of $100 \mathrm{meV}$. Electrons traversed a $23.75 \mathrm{~cm}$ flight path shielded by copper and mu metal. They were detected by a chevron microchannelplate and the time-of-flight distribution was recorded by a transient digitizer, since 5 to 10 electrons were collected per laser pulse. Signal ringing proved to be a problem, since it could not be blocked by a discriminator without loss of signal and distortion of the time-of-flight distribution. ${ }^{128}$ These researchers solved this problem by using their computer to apply a fast discriminator emulation algorithm to the raw data array received from the transient digitizer following every laser shot. Suppression of ringing left clean, sharp peaks corresponding to the leading edges of each microchannelplate pulse that was originally detected. only after being processed in this way was each sweep of the transient digitizer summed into the time-of-flight data array. The angular resolution in this experiment was $\pm 2.5^{\circ}$. The sample could be rotated relative to the detector axis to 
collect dispersion data. This apparatus was also used to study Si(111) $(2 \times 1)^{104,105}$ and GaAs(110). ${ }^{102}$ subsequently, it was used in a multiphoton photoemission study of Pt(111). 129 In the last case, only photons of energy $1.84 \mathrm{eV}$ were used to excite the sample. Several experiments using this instrument were described in a general article by Boxor. ${ }^{89}$

The time-of-flight technique was used by Kubiak in his study of $\mathrm{Pd}(111) \cdot 38$ The sample was excited by dye laser pulses of about $9 \mathrm{~ns}$ duration. Transient electronic states were probed with 4 to $5 \mathrm{eV}$ photons. The flight path was 1 meter long and was housed in a magnetically shielding tube coated internally with colloidal graphite. The electrons were detected by a chevron microchannelplate with a 50 ohm anode. Due to the low repetition rate of the laser, the delayed coincidence technique using pulse counting and a time-toamplitude converter was of no use. Time-of -flight data was collected by a transient digitizer. The number of electrons detected per laser pulse was not given. The sample was rotated relative to the fixed detector axis to study the dispersion of the transient states which were observed. At a kinetic energy of $1.8 \mathrm{eV}$, the resolution was $30 \mathrm{meV}$. This instrument was also used in a study of $\mathrm{Cu}(111), 39 \mathrm{Ni}(111), 40$ and the (111) face of diamond. ${ }^{106}$

A thoroughly optimized instrument for two-photon photoemission with time-of-flight energy analysis has been used by Haight, silberman and Lilie to study GaAs (110). 103.130 
Using an amplified subpicosecond laser, transient electronic states of the sample were excited at $4 \mathrm{eV}$ and probed at 11 to $13 \mathrm{eV}$. Energy resolution of $33 \mathrm{meV}$ at $7 \mathrm{eV}$ kinetic energy was reported. The flight path was $34 \mathrm{~cm}$ in length and was surrounded by a magnetically shielding tube coated internally with colloidal graphite. The multianode energy analyser was inherently angle-resolving because it acted as a positionsensitive detector of single photoelectrons. The flight region was terminated in a chevron microchannelplate assembly and a position sensitive anode array. A $7^{\circ}$ angular range was subtended by 32 adjacent anodes. Thus a pixel of $0.22^{\circ}$ angle could be resolved. Multichannel discriminators and multichannel time-to-amplitude converters allowed each anode to collect independent photoelectron time-of-flight distributions. This instrument has also been used in a study of Ge layers grown epitaxially on GaAs(110). ${ }^{131}$

For purposes of comparison, a capsule summary of the instrument used in the present experiment is provided here as well. The sample was a Ag(111) single crystal with or without. physisorbed materials on the surface. It was excited by cavity dumped dye laser pulses in the range of 3.8 to $4.6 \mathrm{eV}$ at a repetition rate of 1 or $2 \mathrm{MHz}$. Two-photon photoelectrons were produced by probe pulses between 1.9 and $2.3 \mathrm{eV}$. The pulse durations were about $10 \mathrm{ps}$. The flight path was $13.5 \mathrm{~cm}$ and was enclosed by two cylinders of magnetically shielding alloy coated with colloidal graphite. The detector was a 
chevron microchannelplate. Time-of-flight data was collected using a time-to-amplitude converter. Arrival time of the electrons was measured relative to that of the laser pulses. Typically, 1 electron was collected per 1000 lasex shots, giving a countrate of 1000 per second. The sample was rotated relative to the stationary detector in angle-resolved experiments. The effective collection angle in such experiments was $\pm 1.5^{\circ}$. The narrowness of the photoelectron energy features obtained was limited by the energy levels being probed to about $50 \mathrm{meV}$ at $1.5 \mathrm{eV}$. Uncorrected systematic errors in timing limited the resolution expected in principle to $25 \mathrm{meV}$ at $1.5 \mathrm{eV}$. Assuming elimination of these errors, resolution would have been $10 \mathrm{meV}$ or less between 0.3 and $2.5 \mathrm{ev}$. In the following sections, a more detaiied description of the time-of-flight energy analyzer is given.

\subsubsection{Detector}

In the present experiment, the detector was a FTD-2003 unit from Galileo Electro-optics. An exploded view of it is shown in Figure 17. It contained a matched pair of type $\mathrm{N}$ microchannelplates with a $12^{\circ}$ bias angle and $2.5 \mathrm{~cm}$ diameter active area positioned in a chevron configuration. The channel diameter was 10 microns. The bias currents of the plates were matched to within $10 \%$. As a result, it wes not necessary for an external voltage divider to distribute the bias potentials between the plates. The front of the first 
Caption for Figure 17:

Exploded view of the microchannel plate assembly. Fasteners are omitted for the sake of simplicity. Bias voltages are fed to the internal parts shown here by metal strips which run out to the perimeter of the assembly. Fine wires may be used to supply the bias voltages since the currents carried are very small. Connections are llade by spotwelding. 


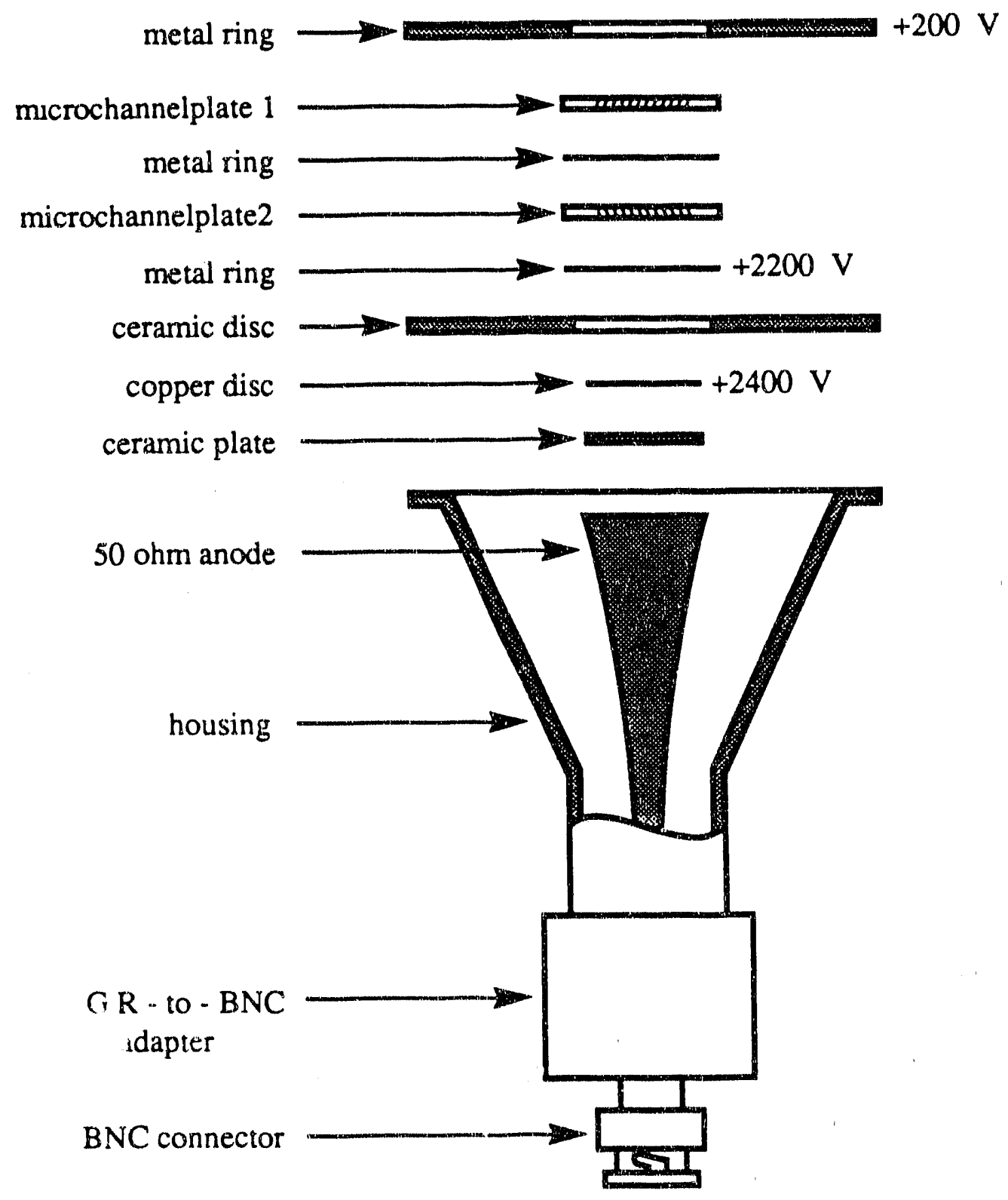

Pigure 17 
plate was placed $0.4 \mathrm{~cm}$ behind a stainless steel mesh of $90 \%$ transmittance which was coated with colloidal graphite. An external power supply (Ortec 459) provided a bias of 2400 volts which was distributed in three steps: A zener diode biased the first plate $+200 \mathrm{~V}$ relative to the grid. The microchannelplate pair experienced a $+2000 \mathrm{~V}$ drop from front to back, and the anode was biased +200 volt relative to the rear plate by another zener diode. The bias current was 15 microamperes. A diagram of the time-of-flight detector showing the electrostatic potentials is given in Figure 18 .

The anode consisted of three parts. Electrons exiting the microchannelplate pair impinged directly on a copper disc which was DC coupled to the high voltage supply through a $10^{6}$ ohm load. This disc was actually a thin deposit of copper evaporated on a $0.5 \mathrm{~mm}$ thick flat ceramic plate which was cemented to a tapered aluminum stalk supported inside of and coaxially with the conical, grounded housing of the microchannelplate assembly. The aluminum stalk was the 50 ohm anode, and, due to the insulating ceramic plate, was isolated from the DC high voltage. It terminated in the center pin of a special, UHV-compatible General Radio type coaxial RF connector at the back of the microchannelplate assembly. This was converted to a BNC connector by a UHV compatible adapter supplied by Galileo. The signal was fed out of the chamber by a Ceramaseal BNC male-male feedthrough with 50 ohm impedence. This feedthrough was welded into a 2.125 inch OD conflat type 
Caption for Figure 18:

Electrical schematic of the two-photon photoemission experiment with time-of-flight detection. The sample is grounded to the chamber by one thermocouple lead. The magnetic shield and grid are biased by batteries and a variable voltage divider. The upper end of the magnetic shield extends to the plane of the sample surface, and the lower end of the magnetic shield envelops the microchannelplate assembly below. The microchannelplate pair is biased by $1000 \mathrm{~V}$ per plate, and a $200 \mathrm{~V}$ collecting bias exists at the input side. A collecting bias is also applied to the copper disc which is separated from the conical anode by a ceramic plate. 


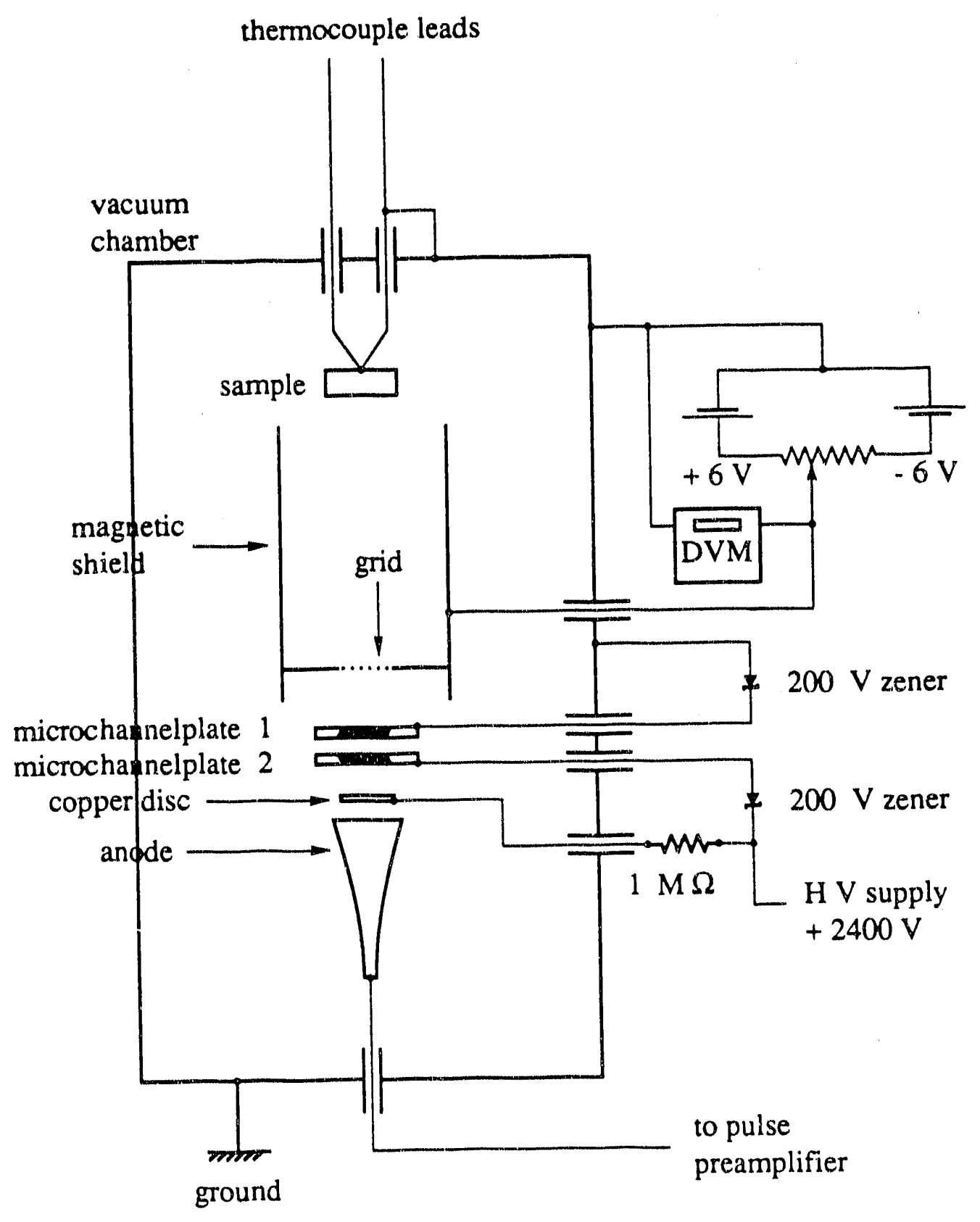

Figure 18 
flange and also provided mechanical support for the microchannelplate assembly. The 2.125 inch flange was supported in the center of a 8 inch $O D$ conflat type flange, which connected directly to the UHV chamber. This flange also supported the grid and cylindrical magnetic shields, as well as six SHV feedthroughs which provide the necessary bias voltages to the microchannelplate.

The grid was a plate isolating the electron multiplier from the field-free flight region. It was made from flat 0.063 inch thick nickel-based magnetic shielding alloy. The same alloy was used in constructing the cylindrical shields around the flight path. It had been annealed after machining to restore maximal permeability. A hole in its center through which the photoelectrons passed and struck the microchannelplates determined its collection angle. The upper bound upon the useful size of this hole was provided by the one inch diameter of the microchannelplates' active region. In order to achieve as high a count rate as possible while retaining the needed angular resolution, the hole was a rectangle, oriented with its long axis parallel to the axis about which the sample rotated. The long dimension was 0.700 inches, so that an angle of $\pm 4^{\circ}$ was subtended horizontally. The short dimension was 0.280 inches, so that an angle of $\pm 1.5^{\circ}$ was subtended in the vertical direction. It was along this direction that the sample normal moved during angleresolved experiments. 
The larger collection angle of $\pm 4^{\circ}$ horizontally was permissible because the derivative of the image potential states' dispersion relation is direction-dependent. The dispersion relation can be regarded as a parabolic surface of revolution about an axis normal to the crystal surface. The direction along which angle resolution is more critical is aligned with the gradient of this surface. Thus the smaller dimension of the rectangular hole lies along this direction. Perpendicular to the gradient, the curvature is much closer to zero. A greater range of the dispersion can be averaged over along this direction without introduction of an unacceptably large uncertainty in the energy. The full active area of the microchannelplate was taken advantage of to do this, giving the $\pm 4^{\circ}$ collection angle along the horizontal direction.

The timing signal from the microchannelplate triggered a Tennelec 454 constant fraction discriminator. A Phillips electronics $2 \mathrm{GHz}$ amplifier with gain equal to 10 was placed before the discriminator. The dark count rate was less than 10/sec. Blocking width was adjusted on the discriminator to minimize spurious peaks due to reflections. The timing output from this discriminator served as the start signal for a Canberra 2043 time-to-amplitude converter. The stop signal was derived from the laser pulses. About 5\% of the laser output was directed onto a DT-110 photodiode which triggerred an ortec 583 constant-fraction discriminator, whose timing signal was directed to the time-to-amplitude converter. The 
time-to-amplitude converter was thus operated in reverse mode. 8192 channels were sorted, and time resolution due to bit noise was $0.061 \mathrm{~ns}$. Scatterred light from the sample produced a small, sharp feature in the time-of-flight distribution which served as a convenient marker of zero flight time. The width of this feature can be taken as a measure of the instrument's temporal response function and was $300 \mathrm{ps}$ or better.

\subsubsection{Jacobian Effect}

Care must be exercised in performing the transformation from the time of flight obtained as raw data to the kinetic energy distribution which is ultimately desired. Calling the time distribution $n(t)$ and the energy distribution $n^{\prime}(E)$, one requires that the integrals of the two be equal to the same number, the total number of counts (N) collected in the experiment:

$$
\int_{t=0}^{t \max } n(t) d t=N=\int_{E_{\min }}^{E=\infty} n^{\prime}(E) d E
$$

Here, $E_{\min }$ is the lower cutoff energy in the energy distribution. It is related to the longest flight time collected by

$$
E_{\min }=\frac{1}{2} m_{e} I^{2} t_{\max }^{-2}
$$


for the electron mass $m_{e}$ and path length 1 .

Formal differentiation of the right and left hand sides of equation (42) and rearrangement gives

$$
n^{\prime}(E)=n(t)\left(\frac{t^{3}}{2 m_{\theta} I^{2}}\right)
$$

The significance of this result is that the number of counts in a given flight time bin must be multiplied by the factor in parentheses to obtain the correct number of counts in the corresponding kinetic energy bin. This factor is the Jacobian in the time-to-energy transformation. It is not merely a constant because the bin size in the energy distribution is not constant but varies smoothly from one end of the distribution to the other. This effect is the same one that causes a given absolute error in flight time to cause a small absolute error in energy at low energies but a large absolute error in energy at high energies.

\subsubsection{Error Analysis}

Error analysis used in the time-of-flight method can be derived in the usual manner 132 and is also treated specifically in the experimental literature. ${ }^{127}$ Error in the energy arises from error $\delta l$ in the path length 1 travelled by the electron as well as from error $\delta t$ in the flight time $t$. calling the absolute error in kinetic energy $\delta E$, 


$$
\frac{\delta E}{E}=\left[\left(2 \frac{8 I}{I}\right)^{2}+\left(2 \frac{\delta t}{t}\right)^{2}\right]^{\frac{1}{2}}
$$

In the present experiment, the path length error $\delta l$ was determined trigonometrically by the photoelectron collection angle $\theta$. For small angles, the relation below gives a good estimate of the relative error in pathlength:

$$
\frac{81}{1} \approx 1-\cos \theta
$$

For $\theta=1.5^{\circ}$, the relative error in pathlength would be $3 \times 10^{-4}$. For $\theta=4^{\circ}$ it was $2 \times 10^{-3}$. The latter value was used in the error analysis described below.

The timing error $\delta t$ arose from three sources. First, the instrument response function of the microchannelplate had a width of $0.1 \mathrm{~ns}$. Second, error existed in the location of the scattered light peak which was used to label zero time in the time-to-energy transformation. This error arose because the nonvanishing propagation time of scatterred light from the sample to the detector was not accounted for. For the $13.5 \mathrm{~cm}$ distance, this error was 0.3 ns. Third, an error was engendered by ignoring the transit time of electrons from the grid, which defined the end of the free-flight region, to the front of the first microchannelplate. The magnitude of this error may be calculated. A $200 \mathrm{~V}$ collecting bias across a 0.4 
cm gap behind the grid gave an electric field of $5 \times 10^{4} \mathrm{~V} / \mathrm{m}$. The force on the electron was its charge multiplied by this field strength. Its acceleration was thus about $10^{16} \mathrm{~m} / \mathrm{sec}^{2}$. Ignoring its initial velocity, its transit time across the gap can be calculated as about $1 \mathrm{~ns}$. It should be noted that the first of these three errors was random, but the latter two were systematic and additive. They both had the effect of making the the flight time seem longer than it actually was. The sum of these three errors gave a total value of $\delta$ t of approximately $1.5 \mathrm{~ns}$. Using equation (45), the error in energy at various kinetic energies can be determined. A list of such values is given in Table II. In rable II, an angular acceptance of the detector equal to $\pm 4^{\circ}$ was assumed, giving $\delta 1 / 1=0.002$.

Because of the nonlinear transformation of flight time to kinetic energy, the fixed error in flight time gives a dramatic scaling of absolute error in kinetic energy with total kinetic energy. The functional form of this dependence is useful to define explicitly. Absolute error in energy $\delta E$ attributable to timing error $\delta t$ is just

$$
\delta E=\left(\frac{\partial E}{\partial t}\right) \& t
$$

where

$$
E=\frac{1}{2} m \cdot l^{2} t^{-2}
$$


Table II Errors in time-of-flight analysis

\begin{tabular}{|l|l|l|l|l|}
\hline $\begin{array}{l}\text { Kinetic } \\
\text { energy } \\
(E),\end{array}$ & $\begin{array}{l}\text { Flight } \\
\text { time } \\
(t) \\
\text { nsec }\end{array}$ & $\begin{array}{l}\text { Rel. } \\
\text { time } \\
\text { error, } \\
\delta t / t\end{array}$ & $\begin{array}{l}\text { Rel. } \\
\text { energy } \\
\text { error, } \\
\text { s. },\end{array}$ & $\begin{array}{l}\text { Abs. } \\
\text { energy } \\
\text { error, } \\
\delta E_{\text {eV }}\end{array}$ \\
\hline 0.1 & 720 & 0.002 & 0.006 & 0.0006 \\
\hline 0.2 & 509 & 0.003 & 0.007 & 0.014 \\
\hline 0.5 & 322 & 0.005 & 0.011 & 0.006 \\
\hline 1.0 & 228 & 0.007 & 0.015 & 0.015 \\
\hline 1.5 & 186 & 0.008 & 0.017 & 0.025 \\
\hline 2.0 & 161 & 0.009 & 0.018 & 0.036 \\
\hline 2.5 & 144 & 0.010 & 0.020 & 0.050 \\
\hline 3.0 & 132 & 0.011 & 0.022 & 0.066 \\
\hline 5.0 & 102 & 0.015 & 0.030 & 0.150 \\
\hline 10.0 & 72 & 0.020 & 0.040 & 0.400 \\
\hline 20.0 & 51 & 0.030 & 0.060 & 1.20 \\
\hline
\end{tabular}

$$
t=\sqrt{\frac{m l^{2}}{2}} E^{-\frac{1}{2}}
$$

thus,

$$
\delta E=\delta t \cdot(\text { const }) \cdot E^{\frac{3}{2}}
$$

As a result of the scaling exhibited in equation (50), error is expected to blow up by 11 times with a fivefold increase in kinetic energy, and by 32 times with a tenfold increase in 
kinetic energy.

2.5 Contact Potential Difference

Time-of-flight analysis of photoelectrons from solids is sensitive to contact potentials existing between the sample surface (Ag(111) in this case) and the material existing at the surface of the detector (colloidal graphite). When they are electricaliy connected, as they must be to provide a definable reference potential and avoid charging, current flows between the two. It is driven by the difference in their Fermi energies. The Fermi energy thus behaves as a thermodynamic chemical potential for flow of free charges. Charge accumulates on the surfaces of tine two bodies, and its distribution is determined by the geometry of the system. The material of lower work function acquires a net positive charge, and the magnitude of the resulting electrostatic potential difference is the difference in work functions of the two materials. As a result of the surface charge, the Fermi. levels inside the materials become equal, and an electrostatic field is established in the volume of space near the two. This situation is diagrammed in Figure 19.

The electrostatic field due to contact potential difference presents a real problem for time-of-flight phototelectron energy analysis. Photoelectrons are accelerated or decelerated by the field, which is not homogeneous. If their flight times are measured, their 
Caption for Figure 19:

contact potential diagram for the time-offlight detector and clean silver(111) sample. Evacum is the energy of a stationary electron outside one of the bodies, beyond the range of the image potential. $\varepsilon_{\text {fermi }}$ acts as a chemical potential for electron flow between the two bodies. $\phi$ is the work function. In (a), the sample is held at ground potential, and the detector floats electrically. In (b), the two have been electrically connected. surface charge has accumulated, driven by the electronic chemical potential. An electrostatic field is established, causing the vacuum energy to become ramplike. The corresponding voltage drop is the contact potential difference. In (c), a DC bias has been judiciously applied to null the contact potential and the net surface charge on each body. 
a)

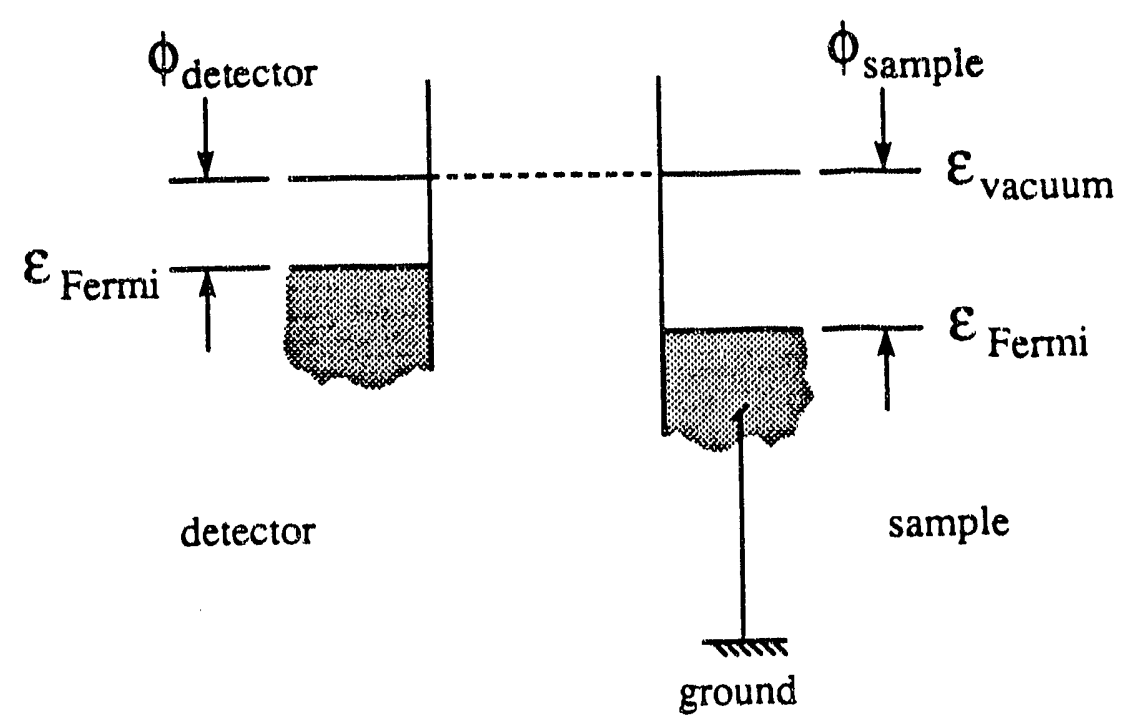

b)

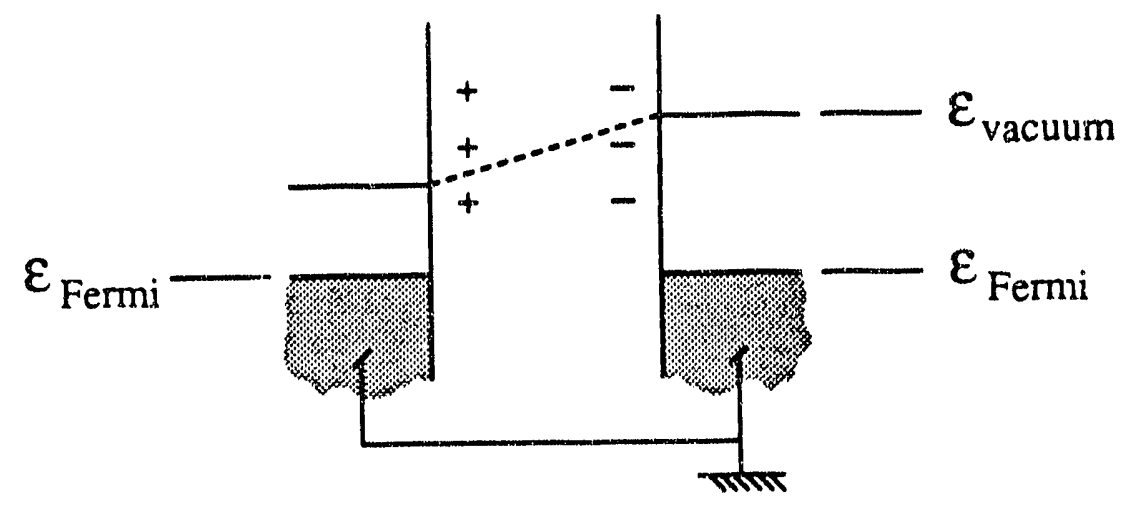

c)

Pigure 19

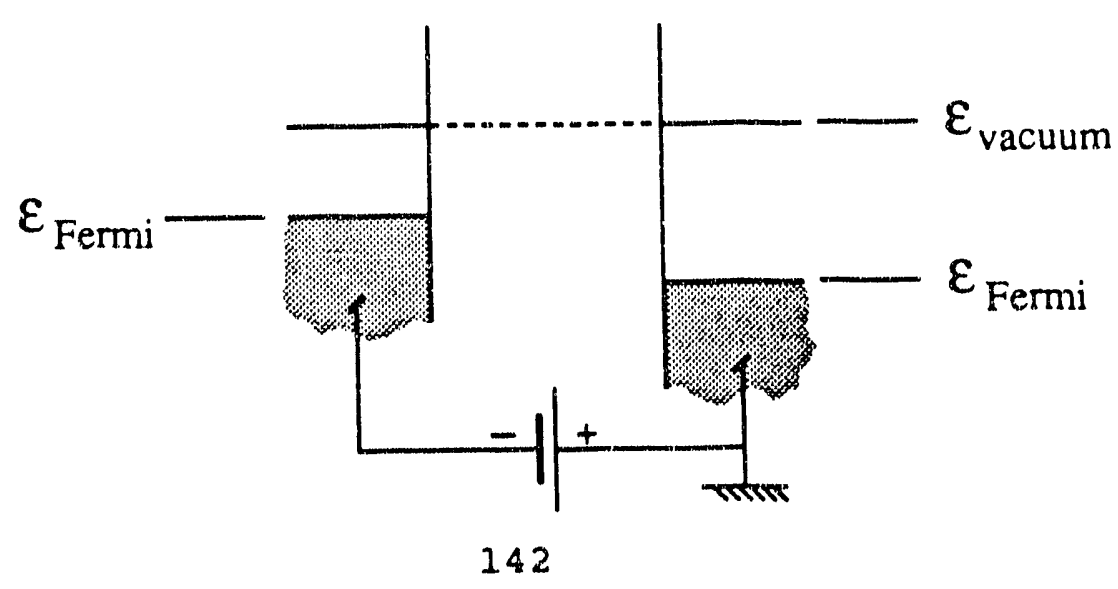


initial kinetic energy can be determined by knowing the exact functional form of the electric field configuration in the flight region and integrating Newton's equation of motion over the whole trajectory. Although simple in principle, this procedure is not to be adopted in a real experimental situation.

The approach used in the present experiment was to apply an accurately measured DC bias between the sample and the detector. From the point of view of the photoelectrons, the detector was actually the grid and cylindrical snields, which were all coated with colloidal graphite. This assembly was referred to as the flight tube. The bias was adjusted to make the measured kinetic energy of the photoelectron feature assigned to the $n=1$ image potential state on $A g(111)$ equal to the difference between the energy of the photoemitting photon and the binding energy of $0.77 \mathrm{eV}$ measured by Giesen and coworkers. 90 This bias was applied between the flight tube and the chamber (ground) by a floating, battery-powered variable voltage divider. It was measured by a $31 / 2$ digit batterypowered digital multimeter. The sample was clipped to ground outside the chamber by one of its thermocouple wires. on clean Ag(111), a bias of $-0.400 \mathrm{~V}$ applied to the flight tube gave the required kinetic energy for the $n=1$ image potential state peak. This result implies the work function of the colloidal graphite coating the flight tube was lower than that of the sample by $0.400 \mathrm{~V}$. 
Deposition of adsorbate samples on the sample changed its work function and necessitated readjustment of the bias applied to the flight tube. All adsorbates used in this experiment had the effect of lowering the metal's work function. Thus, the new bias needed at the detector moved in the positive direction, closer to ground potential. Because a change in the binding energy of the $n=1$ image potential state could have accompanied the adsorbate-induced work function shift, the bias was no longer determined from the position of the $n=1$ peak. Instead, the change in the work function was measured independently by a threshold photoemission experiment.

In the threshold photoemission experiments, a Hamamatsu super quiet type deuterium lamp served as a spectrally smooth source of UV light. It was monochromated by a Jarrell-Ash $1 / 4$ meter monochromator, resulting in a tunable, narrowband source of photons with energies continuously variable between 3.10 and $4.95 \mathrm{eV}$. The bandwidth of the monochromated light was about $50 \mathrm{meV}$. The monochromator was scanned by a Klinger stepper motor interfaced through a GPIB bus with an IBM PC compatible computer. Uncoated aluminum mirrors and UV grade fused silica lenses were used to steer and focus the UV beam onto the sample. Photoelectrons from the sample were collected by the time-of-flight detector with a $+70 \mathrm{~V}$ bias. Time of flight was not analyzed. The overall countrate was recorded by the same computer which scanned the monochromator. 
counting was done by a counter-timer input on the GPIB board. This board was a Power -488 model from Iotech. Data collection was managed by the $C$ program called RMAIN which was written ad hoc. Countrates up to $10^{4}$ per second and steplike but. rounded photoelectric threshold spectra were typically obtained.

The functional forms of these thresholds were, unfortunately, not well fit by Fowler's function, ${ }^{133}$ probably due to a strong dependence of illumination intensity upon photon energy. Nonideal behavior may also have resulted from variation in the optical properties of silver 119 over the photon energy range of interest. A good value for the absolute work function of the samples could not be obtained. Instead, the best value from the literature $(4.56 \mathrm{ev})^{86}$ was taken as the work function of the clean Ag(111) samples. Workfunctions of the samples containing adsorbates were then measured relative to this value by okserving changes in the threshold photoemission spectra. Relative work functions were determined by reading the photon energy corresponding to a point on the threshold photoemission spectrum a given fraction of the way down its declining edge. Estimated uncertainty in this procedure was $\pm 50 \mathrm{meV}$.

It is worthwhile to compare briefly the time-of-flight energy analysis technique with other methods of electron energy analysis. Most other analyzers act as electron monochromators by subjecting the incoming flux of electrons to a pair of curved electrodes held at different potentials. 
These are referred to as electrostatic deflector type analyzers. The various geometries commonly used are described in several review articles.134,135,136,137 Entrance and exit apertures at each end of the electrodes, combined with the deflection the electron's trajectories experience between the electrodes, ensure that only electrons of a specific kinetic energy are transmitted through the analyzer. Typically, an electron multiplier operating in either its pulse-counting or current modes detects the transmitted electrons. With such devices, only a single energy channel is analyzed at any time, so the great majority of the electrons emitted by the sample are wasted. The electrons' kinetic energy distribution must be collected by scanning the pass energy, a very tedious procedure if a large scan must be collected at high resolution. In contrast, the time-of-flight technique as implemented in the present work collects all energy channels in parallel. Collection of the electron energy distribution is completely efficient even at very high resolution. No electrons emitted into the collection angle defined by the detector aperture are wasted. This is the most important advantage of the technique.

The time-of-flight technique has two chief disadvantages. First, it generally requires pulsed excitation of the sample, although Tsai, Baer and Horovitz ${ }^{125}$ appear to have had some success in circumventing this requirement. Pulsed lasers lend themselves naturally to experiments involving time-of-flight 
analysis. The synchronously pumped dye laser used in the present work, with its several-picosecond pulses, is particularly well suited to the time-of-flight analysis scheme. Longer laser pulses require longer flight distances. This effect is evident in the apparatus of Kubiak. ${ }^{38}$ The second disadvantage of the technique is its precipitous drop in energy resolution above a few ev kinetic energy. This behavior is illustrated in Table II on page 139. Even a very small error in flight time produces large exrors in kinetic energy. At kinetic energies of several ev or more, deflector type analyzers are necessary if high energy resolution is required. 


\subsection{Clean Silver (111)}

Thorough examination of the properties of the image potential states on clean $\mathrm{Ag}(111)$ was necessary to interpret results obtained from two-photon photoemission experiments on this surface when covered by adsorbate layers. A wavelength survey was performed to map out the various two-photon excitations which can be observed. Attention was then focussed on the spectral feature assigned as the $n=1$ image potential state, and the dispersion relation of this state was measured. Comparison of this result to those of other workers revealed the performance of which the new two-photon photoemission spectrometer is capable and established a scientific base which was essential to judge the signficance of novel results on metal-dielectric systems. In addition, the experimental data were compared to a model of the image potential state based on multiple reflection theory in order to demonstrate the applicability of this formalism to the present experimental work.

Raw two-photon photoemission data consist of a distribution of flight times as shown in Figure 20. This figure represents a total collection time of two minutes. The largest feature is due to excitation of the $n=1$ image potential state by UV light, followed by photoemission induced by the visible wavelength. This feature is conveniently 


\begin{abstract}
Caption for Figure 20:
Time-of-flight distribution of two-photon photoelectrons from clean silver(111). These photoelectrons were emitted normal to the surface. Image potential states are excited by one UV photon. Photoemission by a visible photon produces the feature labeled "UV+Vis." Photoemission by a second UV photon produces the feature labelied "UV+UV." The smallest feature is due to scatterred UV light striking the electron multiplier.
\end{abstract}




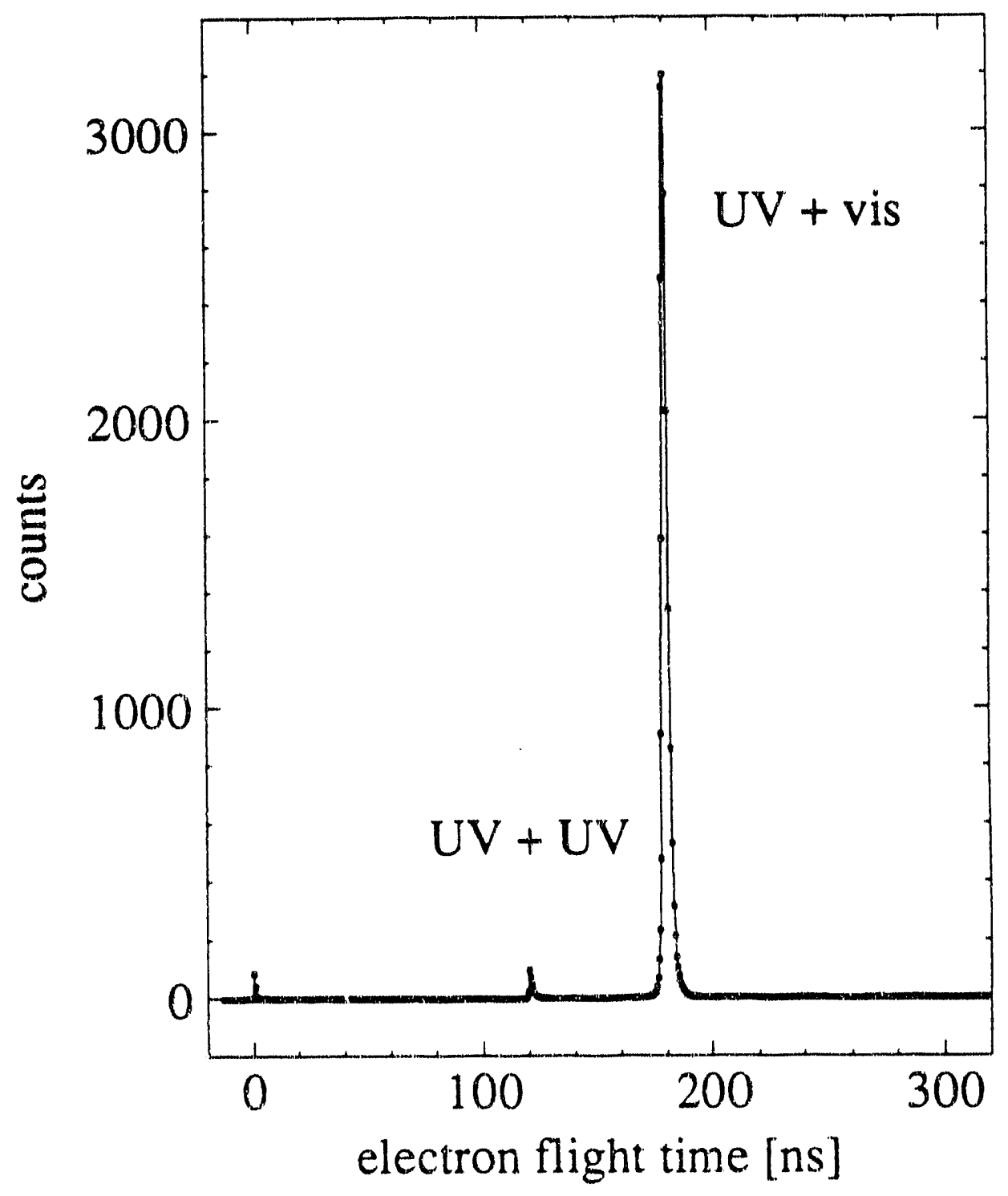

Figure 20

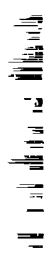


referred to as a UV-Visible peak. The smaller feature is due to photoemission of the image potential state by a second UV photon. Thus it may be referred to as the UV-UV peak. The smallest feature is due to UV light scatterred by the sample and detected direcly by the electron multiplier. It conveniently labels the zero of the flight time axis to an accuracy limited by the travel time of light across the flight distance of $13.5 \mathrm{~cm}$. In this data, one point represents 0.5 ns. Thus, the width at half height of the prominent UVvisible peak is about $3 \mathrm{~ns}$.

The width of the scatterred light feature is a measure of effective instrument response time. It is about $1 \mathrm{~ns}$, rather than $0.1 \mathrm{~ns}$, which would be expected for a fast microchannelplate-based detector. When the scatterred light peak is viewed under very high resolution, this broadening is seen to be due to a second, smaller peak accompanying the main feature. This is probably a reflection due to a discontinuity in electrical impedance at some point in the timing circuit. It could be minimized by careful adjustment of the discriminator settings. Since the error it contributed to the energy measurement is only on the order of several mev, its occasional appearance was typically tolerated.

Transformation of the time distribution to a distribution of photoelectron kinetic energies is shown in Figure 21 . The large peak is again the UV-visible two-photon photoelectron excitation attributable to the $n=1$ image potential state. Its 
Caption for Figure 21:

Distribution of kinetic energies of two-photon photoelectrons emitted normal to the surface. clean silver (111) is the sample. The largest feature is due to photemission of the image potential state by a visible photon. The smaller feature is due to photoemission by a UV photon. 


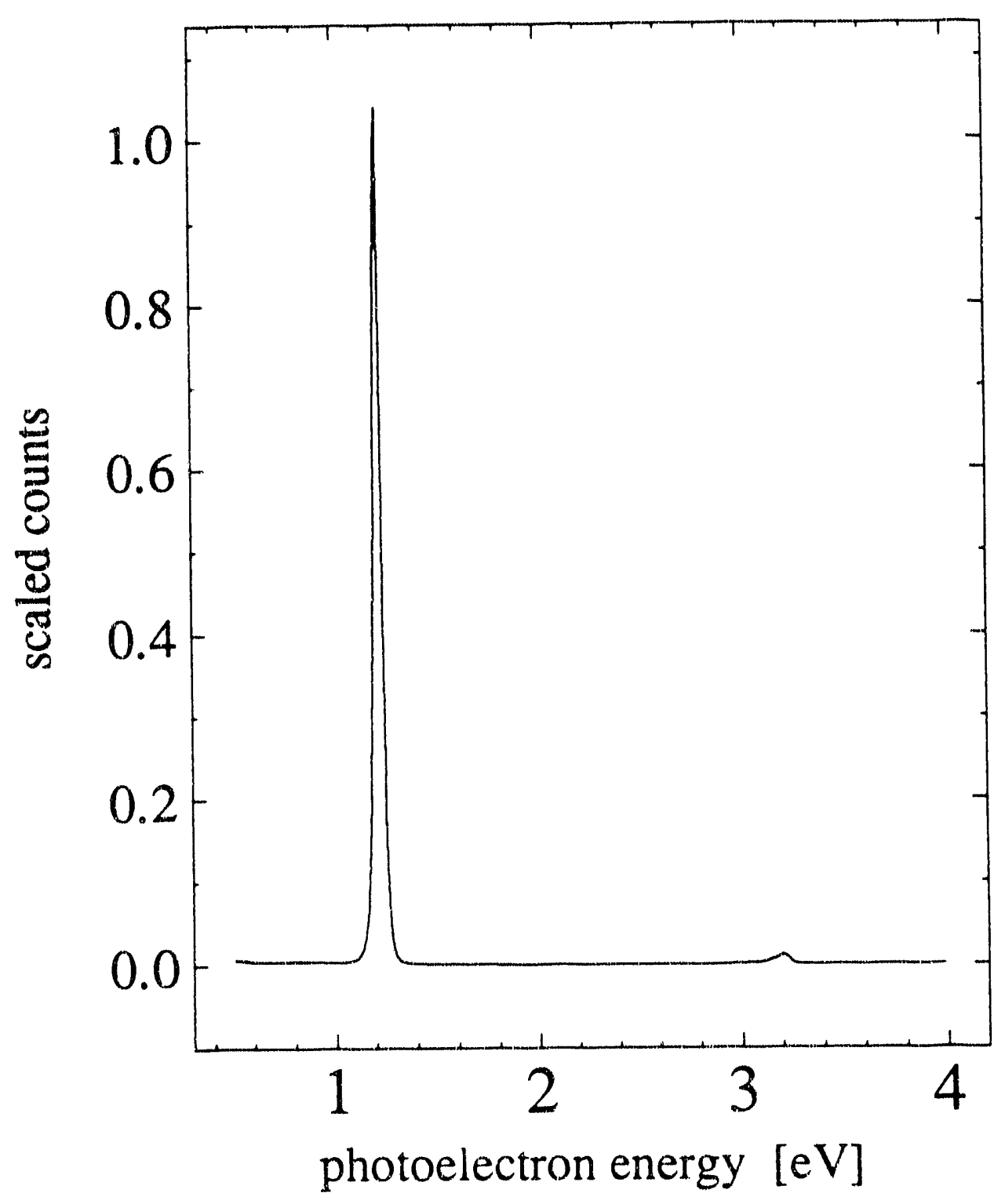

Plgure 21 
width is about $30 \mathrm{meV}$. The peak at high energies is the UV-UV excitation and is now smaller in comparison to the UV-visible peak due to the effect of the monotonically decreasing Jacobian function at shorter flight times. The spectrum is truncated to the low-energy side of $0.5 \mathrm{eV}$ because the Jacobian diverges there, exaggerating the presence of useless, low-energy noise and scatterred electrons.

The variety of two-photon photoelectron excitations which is possible on the Ag(111) surface is indicated by the data in Figure 22. This is a set of photoelectron kinetic energy distributions collected at a series of laser wavelengths throughout the tuning range of the dye laser. Photoelectrons emitted normal to the crystal surface were detected, and only spectral features due to UV-visible excitations are shown. Three possible excitation mechanisms are shown in Figure 23. The prominent series of peaks whose energies depend less strongly upon photon energy are assigned to the $n=1$ image potential state. This state is initially excited from the continuum of bulk levels below the Fermi energy. As the exciting photon energy is increased, lower lying bulk levels are able to be excited. Since the $n=1$ image potential state constitutes an intermediate state of fixed energy (at zero wavevector in the plane of the surface), incremental increases in the photoelectron kinetic energy as photon energy increases are numerically equal to incremental increases in energy of the photoemitting (visible) photon. In this sense, the slope 
Caption for Figure 22:

Two-photon-photoelectron kinetic energy distributions at a variety of laser wavelengths. clean silver (111) is the sample. The image potential state is actually excited by UV light, which is the second harmonic of the wavelength indicated in this figure. UV photon energies thus range from $3.76 \mathrm{eV}$ to $4.39 \mathrm{eV}$. Over the range of kinetic energies shown, all photoelectrons are due to photoemission by the visible light, which ranges from 1.88 to $2.20 \mathrm{eV}$ photon energy. Different dependence of kinetic energy upon incremental increases in photon energy distinguishes the $n=1$ image potential state feature from the feature due to nonresonant excitation of the occupied surface state. The $n=2$ state is excited by UV photons of the highest energy. 


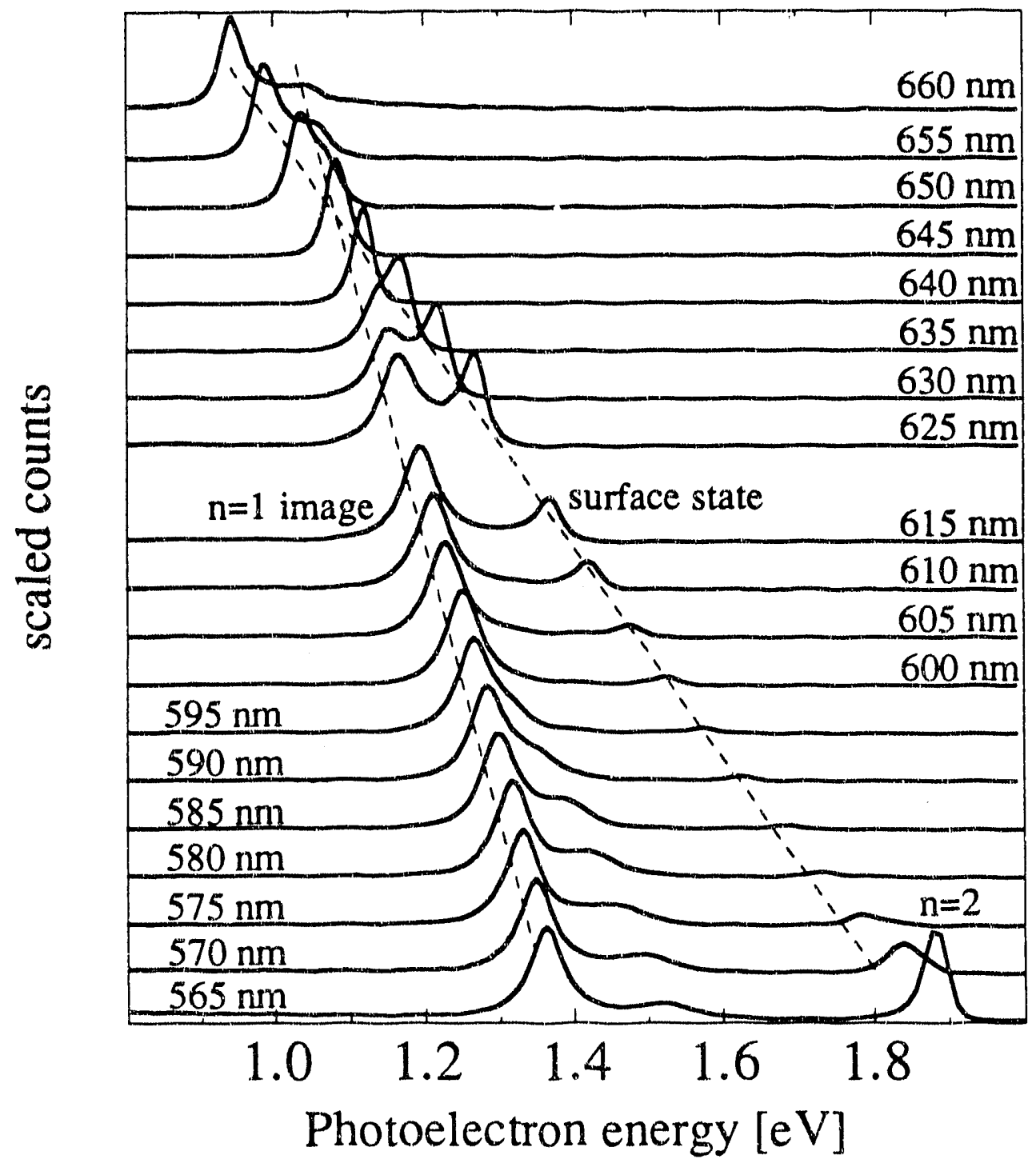

Pigure 22 
Caption for Figure 23:

Diagram of observable two-photon photoelectron excitations on silver(111). Energy levels are shown here to scale. Shading indicates the continuum of bulk states. In (a), the $n=1$ state is excited from occupied bulk bands, and a photoelectron is ejected from it. In (b), photoemission from the occupied surface $(n=0)$ state occurs through a nonresonant two-photon process. In (c), photoemission occurs from the $n=2$ state, which is populated from the bulk continuum. Photons of higher energy are needed for this excitation. In all cases shown here, the second harmonic of the dye laser output provides the UV light for the first step of the excitation, and the visible fundamental excites the photoelectron. 


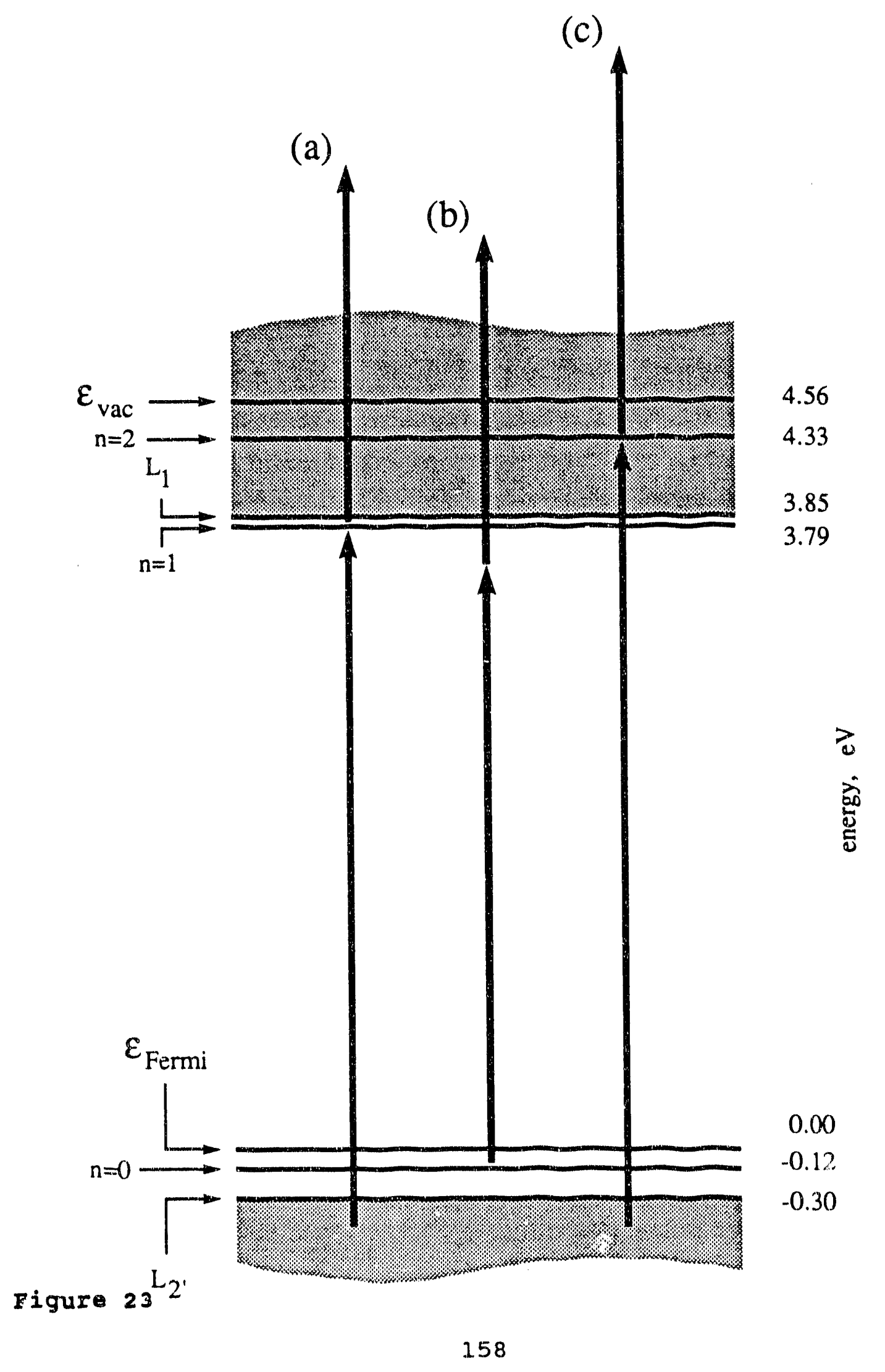


associated with each series of peaks in the wavelength survey is important in assigning their physical origin.

In collecting this data, the flight tube was biased -0.400 volts relative to the sample to offset the difference in work functions. This value of the flight tube bias was selected empirically in order to make the difference between the measured kinetic energy of photoelectrons appearing in the $\mathrm{n}=1$ image potential state peak and the energy of the visible photons equal to $0.77 \mathrm{eV}$. This was the binding energy reported by steinmann ${ }^{50}$ for the $n=1$ image potential state.

In contrast to the $n=1$ image potential state feature, where the energy of the intermediate state in the two-photon photoemission process was constant, a fundamentally different excitation mechanism is responsible for the other prominent series of peaks in Figure 22 whose energies vary more rapidly with changes in photon energy. Here, the initial state is the occupied I-gap surface state. The photoelectron peak is due to nonresonant two-photon photoemission of the surface state. In this case, the energy of the intermediate state is not constant since it is a virtual state. Incremental increases in the energy of both photons add to give the resulting photoelectron kinetic energy, with the UV photon contributing twice the energy increment of the visible. As a result, the kinetic energy varies as three times the energy of the visible photon.

An alternate method of reckoning the bias between sample 
and flight tube is to use the occupied I-gap surface state instead of the image potential state as the reference feature. Picking any one of the wavelength scans, the sum of UV and visible photon energies is subtracted from the photoelectron kinetic energy to give the surface state energy relative to the vacuum level. The work function, taken to be $4.56 \mathrm{ev}, 86$ is then subtracted from this value to give the binding energy of the surface state relative to the Fermi energy. The binding energy obtained from the wavelength data by this analysis is $0.12 \mathrm{ev}$, in good agreement with the literature value of $0.120 \pm 0.010 \mathrm{eV}$ obtained by high resolution ultraviolet photoelectron spectroscopy. 110

Excitation with photons of the highest energies produces a feature assigned in Figure 22 as the $n=2$ image state. Its mode of excitation is the same as that of the $n=1$ state. Its energy relative to the vacuum level is found to be $-0.32 \mathrm{ev}$, in good agreement with the previously measured value of -0.30 eV. ${ }^{90}$ This binding energy places it above the upper edge of the L-gap, degenerate with the continuum of bulk freeelectron-like bands.

The dispersion of the $n=1$ image potential state on clean Ag(111) was determined by angle-resolved two-photon photoemission. Angle-resolved experiments were conducted at a UV photon energy of $4.07 \mathrm{eV}$, high enough for the second harmonic to excite the image potential state fxom the continuum of occupied bulk states below the projected bu. : 
bandgap. At this photon energy, the feature due to nonresonant excitation of the surface state is well separated from the $n=1$ image potential state.

Figure 24 is a set of photoelectron kinetic energy distributions taken at a series of sample rotation angles. Dispersion of the $n=1$ image potential state is evident. The peak becomes broader at large rotation angles because these scans correspond to higher values of momentum in the plane of the surface. Since the acceptance angle of the detector and thus the collection window in $k$ space are fixed, averaging occurs over a greater range of energies on the dispersion curve at large rotation angles. The shoulder to the left of the $n=1$ peak, which is due to nonresonant two-photon photoemission of the occupied surface state, is also seen to be dispersive. It gradually loses intensity as the rotation angle increases. This effect is expected, since the occupied surface state crosses the Fermi level and becomes unoccupied around $0.1 \AA^{-1}$.

Using equation (41) and the positions of the $n=1$ peak maxima, a value for momentum in the plane of the surface was calculated for each scan. Energy is plotted as a function of momentum in the surface plane in Figure 25. A parabolic dispersion relation was fit to this data and, from its curvature, an effective mass of $\mathrm{m}^{*}=(1.4 \pm 0.1) \mathrm{m}_{\mathrm{e}}$ was extracted for the $n=1$ image potential state. This compares well with the value of $(1.3 \pm 0.15) \mathrm{m}_{\mathrm{e}}$ reported in the literature. 50 
Caption for Figure 24:

Kinetic energy distributions of two-photon photoelectrons collected at a series of angles between the sample normal and the detector axis. The $n=1$ state on clean silver (111) is observed. Dispersion of the energy of the image potential state with increasing value of wave vector in the plane of the surface is evident. 


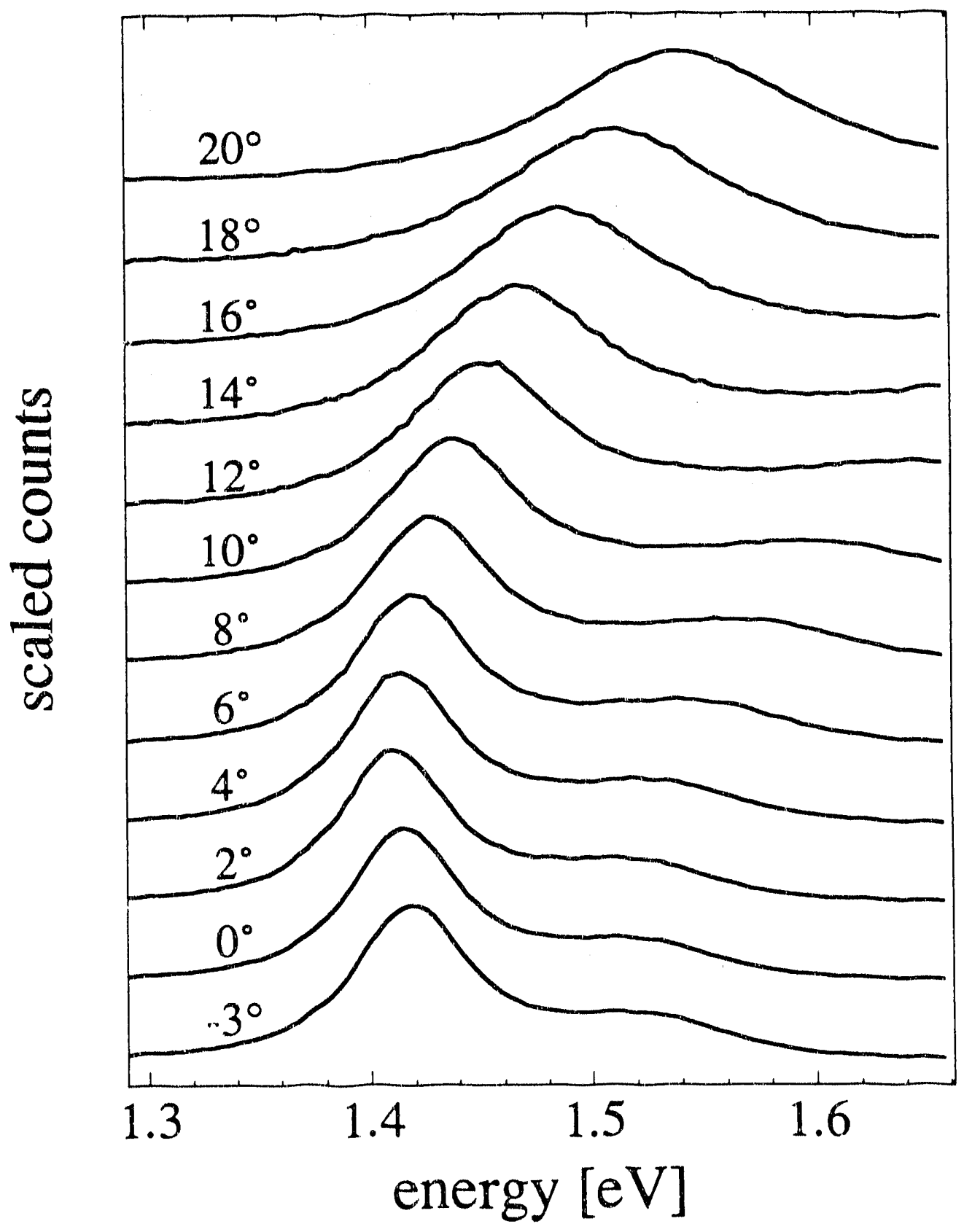

Figure 24 
Caption for Figure 25:

Image potential $(n=1)$ state energy measured relative to its value at the zone center is plotted against wave vector in the plane of the surface. sample is clean silver (111). Points are obtained from a series of angle-resolved scans as in Figure 13. Best fit parabolic dispersion relation is shown. 


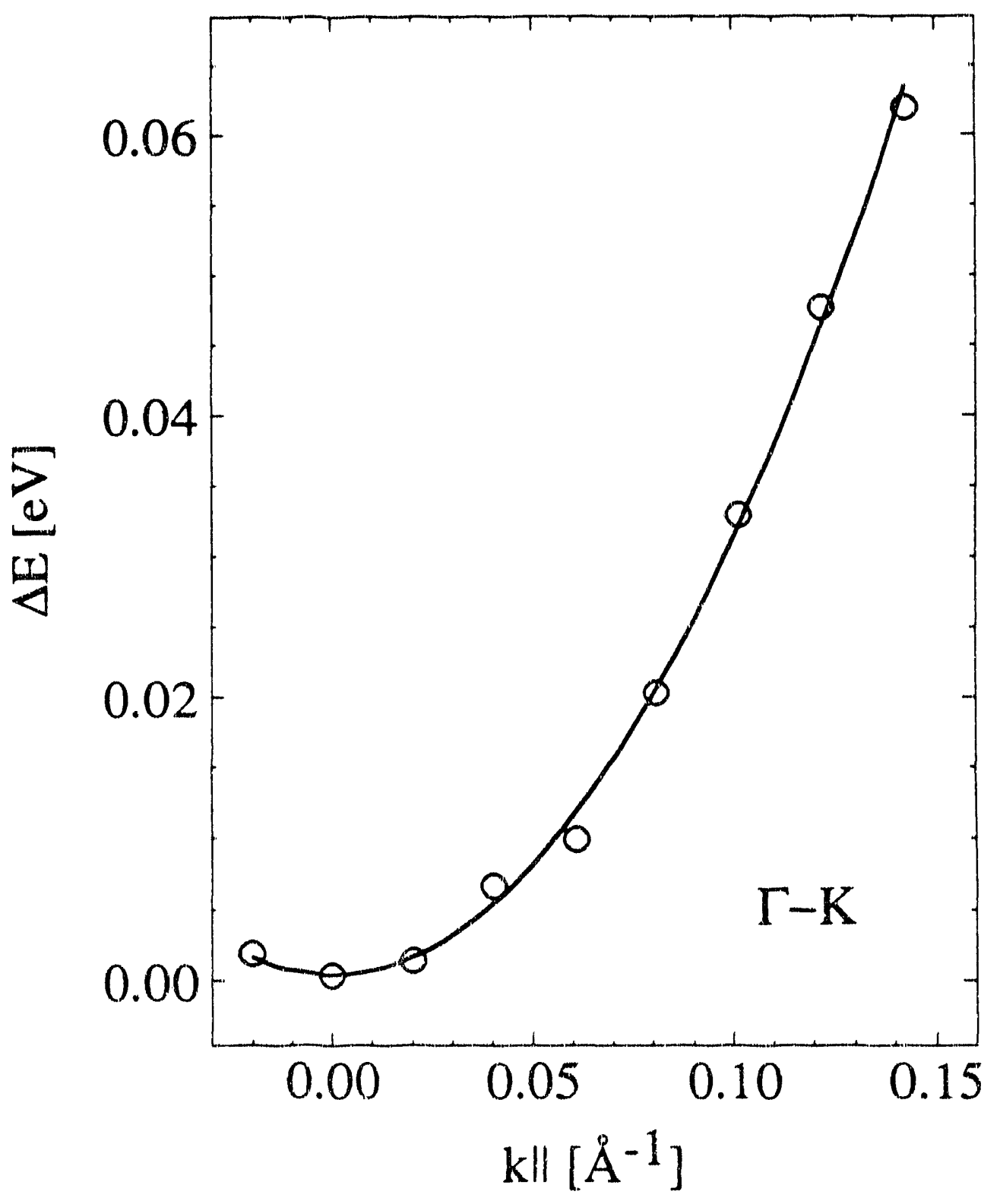

Figure 25

$=$
$=$ 
The $n=1$ image potential state on Ag(111) was modelled by multiple reflection theory, for comparison with the results obtained by two-photon photoemission. This calculation was an important starting point for the subsequent consideration of the effects of physisorbed layers upon the image potential states. The bulk band structure along $\Gamma-L$ was parametrized according to the diagram in Figure 26 . The work function of $\mathrm{Ag}(111)$ was taken as $4.56 \mathrm{ev}, 86$ and the midgap energy was taken as $9.64 \mathrm{ev} .{ }^{26}$ The energy of the gap bottom $\left(I_{2,}\right)$ relative to the Fermi energy was taken as $-0.30 \mathrm{ev}, 110$ and the energy of the gap top $\left(L_{1}\right)$ relative to the Fermi energy was taken as $3.85 \mathrm{eV} .86$ The remainder of the energy parameters used in the calculation were deduced from these values. The bulk bands were assumed to be parabolic but modified by the existence of the gap around $L$. In order to arrange that the bulk freeelectron parabola crossed $\mathrm{I}$ at the midgap energy of $9.64 \mathrm{eV}$, an effective mass of 0.708 was used for it. Thus, the parameter $c^{2}$ was taken to be equal to $5.45 \mathrm{ev} \cdot \mathrm{A}^{2}$ in describing the bulk bands. The zone boundary was taken to lie at 1.33 $A^{-1}$. The fundamental reciprocal lattice vector (g) was 2.66 $\AA^{-1}$, and the interlayer spacing along $\Gamma-L$ was $2.36 \AA$. The image piane was taken at $z=a / 2$, half an interlayer spacing past the last row of ion cores. This was also the location of the plane at which logarithmic derivatives of the wave function inside the crystal and outside of it were set equal. The phase shifts $\phi_{B}$ and $\phi_{C}$ were taken from equations (37) 
Caption for Figure 26:

Map of parameters used in multiple reflection theory calculation for clean silver (111). Energy values in black type are from the literature and are keyed to the reference section of this work by the letters in parentheses as follows:
(a)
Reference 110
(b)
Reference 86
(c)
Reference 26

Energy values in white type on a black background are derived from the referenced quantities. Value (d) denotes the energy at which the imaginary part of the complex wave vector reaches its extremum. The effective mass of the bulk band is such that, if $V_{g}$ were zero, the parabolic dispersion curve would cross $L$ at the value of $v_{g}$ indicated. 


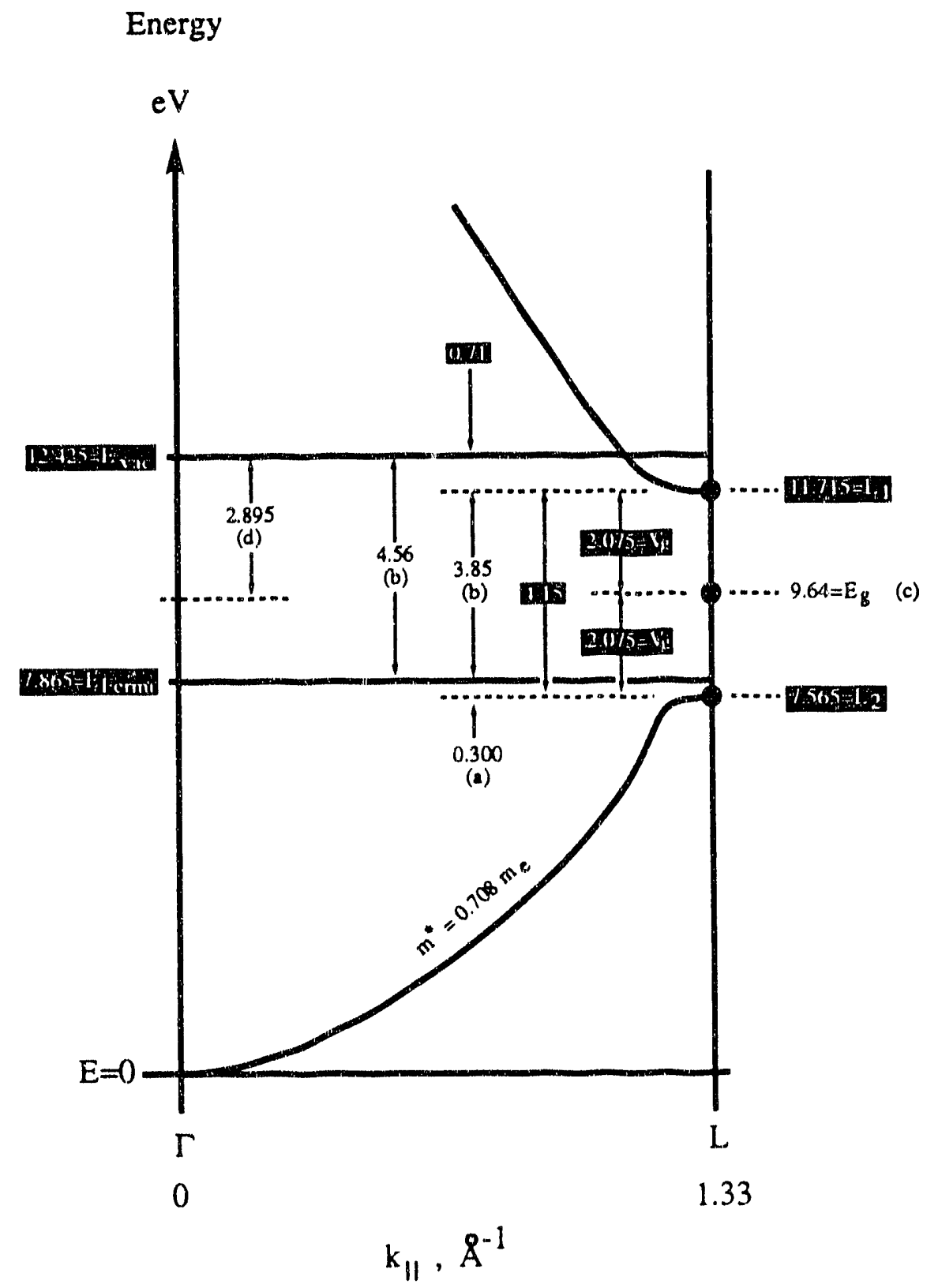

igure 26 
and (35). The parameters $\delta, q$ and $x$ needed to evaluate $\phi_{c}$ were taken from equations (29), (30) and (10). The total phase shift $\phi_{T}$ was calculated simply as the sum of $\phi_{B}$ and $\phi_{C}$ :

$$
\phi_{T}=\phi_{B}+\phi_{C}
$$

Plots of the three terms in this equation are shown in Figure 27. The phase shifts were only evaluated in the energy interval inside the gap, since the phase $\delta$ of the wavefunction tail is strictly defined only for these energies. Near the midgap energy, q reaches its extremum. Different branches of the arcsin function must be used in the definition of $\delta$ above and below this energy. Above it, $\delta$ and $\phi_{c}$ were evaluated using the branch in which the arcsin function assumes the values between 0 and $\pi / 2$. Below this energy, The branch was used in which the arcsin function assumes values between $\pi$ and $3 \pi / 2$.

The $n=0$ eigenvalue was found by setting $\phi_{T}$ equal to 0 and solving numerically for the energy. The eigenvalue was 4.63 $\mathrm{eV}$ below the vacuum energy $(0.070 \mathrm{eV}$ below the fermi energy). Similarly, for the $n=1$ state, $\phi_{T}=2 \pi$, and the eigenvalue was $0.78 \mathrm{eV}$ below the vacuum erexgy. These numbers may be compared to the calculations of Giesen, et al. ${ }^{50}$ who treated Ag(111) by the same method using slightly different parameters for the band structure. For $n=0$, they obtained an eigenvalue $0.07 \mathrm{eV}$ below the Fermi energy, and for $\mathrm{n}=1,0.75 \mathrm{eV}$ below the vacuum energy. The latter number differs from the one 
Caption for Figure 27:

Plots of crystal-induced phase shift, barrierinduced phase shift and total phase shift used in multiple reflection theory calculation for clean Ag (111). 

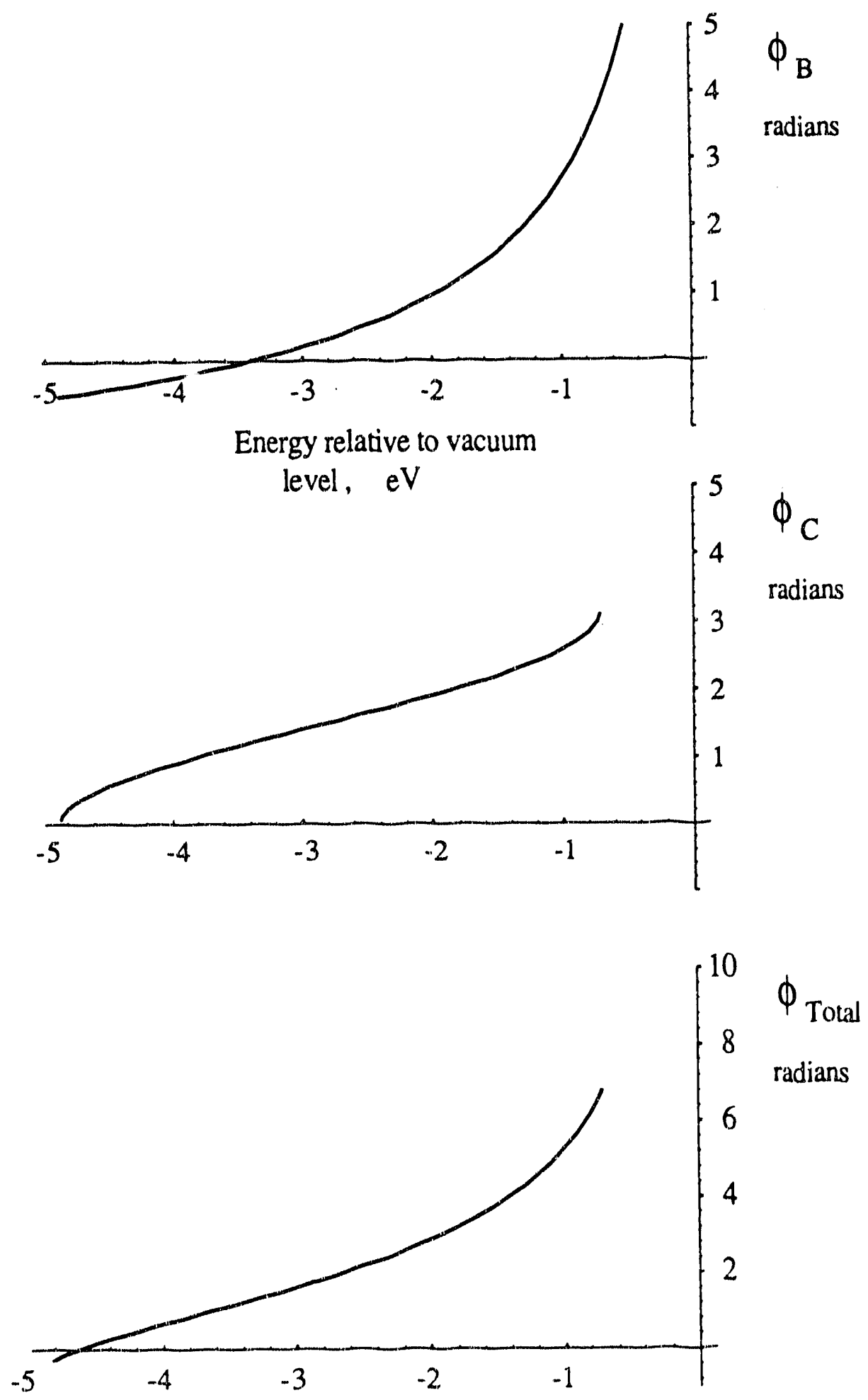

Figure 27 
calculated in the present work chiefly because the upper band edge position was taken to be slightly lower in the work of Giesen, et al.

The difference in upper band edge energies also affects the effective mass calculated by the means of Giesen, et al. ${ }^{50}$ In their calculation, the work function was taken as $4.56 \mathrm{ev}$, and the various parameters necessary in the calculation are listed below:

Table III parameters in effective mass calculation

\begin{tabular}{|l|l|l|}
\hline Feature & $\mathrm{m}^{*} / \mathrm{m}_{e}$ & $\begin{array}{l}\text { Energy at } \\
\mathrm{k}_{\mathrm{j}}=0 \text { relative } \\
\text { to } \mathrm{E}_{\mathrm{wa}}\end{array}$ \\
\hline $\begin{array}{l}\text { upper band } \\
\text { edge }\end{array}$ & 1.60 & -0.600 \\
\hline $\mathrm{n}=1$ state & ---- & -0.752 \\
\hline $\begin{array}{l}\text { free electron } \\
\text { parabola }\end{array}$ & 1.00 & -0.848 \\
\hline $\begin{array}{l}\mathrm{n}=0 \text { state } \\
\text { lower band } \\
\text { edge }\end{array}$ & 0.60 & -4.624 \\
\hline
\end{tabular}


Equation (40) was used to calculate an effective mass of 1.33 for the $n=1$ state. The data in Table III may be adapted to the present calculation by letting the binding energy of the $n=1$ state be $-0.78 \mathrm{eV}$ and the energy of the upper edge be $-0.710 \mathrm{eV}$. Then the effective mass is calculated as 1.44 . Despite the simplified nature of this theory, it does indicate the physically correct conclusion that the properties of the $\mathrm{n}=1$ image potential state on $\mathrm{Ag}(111)$ are strongly determined by details of the upper band edge.

\section{2}

\section{Xenon-Covered Silver (111)}

The culmination of the present work lies in the investigation of dielectric films on metal surfaces. Image potential states in such systems have not been studied before, but were expected to be sensitive to the adsorbate. Xenon physisorbed on $\mathrm{Ag}(111)$ was chosen as the first metaldielectric system for study. Xenon is chemically nonreactive and is known to form an ordered monolayer phase on Ag(111). ${ }^{138,139}$ The work function and bandgap energy of bulk, solid xenon, important parameters in a study such as this are well documented. ${ }^{140,149}$ Another advantage was that its effect upon the work function of $\mathrm{Ag}$ (111) has been examined previously. ${ }^{142}$ When the empirical xenon-xenon interaction potentials have been fit to the Lennard-Jones form, values for the collision parameter $\sigma$ between $4.06 \AA$ and $4.22 \AA$ have resulted. ${ }^{143}$ 
In Figure 28, the effect of varying coverages of xenon upon the $n=1$ image potential state feature is shown. The experiment was performed at a temperature of $25 \mathrm{~K}$. The image potential state on clean Ag(111) was accompanied by a smaller feature attributed to the nonresonant excitation of the occupied I-gap surface state. This appears as a shoulder to the right of the large peak. It was quenched as adsorbate coverage increased. As this occurred, intensity in the $n=1$ image potential state at the position appropriate to the clean surface decreased, and intensity accumulated in a new feature at lower kinetic energy. The new feature was assigned to the $\mathrm{n}=1$ image potential state existing within the boundaries of one-layer-deep islands of physisorbed xenon. Since the new feature is narrow, all the patches contributing to it must provide equivalent environments for the image potential states. In particular, the density of xenon atoms within the patches must be fixed, independent of the mean surface coverage. The relative intensities of the two peaks are taken as an indication of the fraction of total surface area covered by xenon atoms.

This apparent observation of xenon layer growth proceeding by the development of isolated islands is significant, if not surprising. The opposite end of the spectrum of possible growth mechanisms would entail adsorbed xenon atoms remaining very close to the location of their 
Caption for Figure 28:

Langmuir dosing of xenon on silver (111) at 25 Kelvin. Intensity in the $n=1$ state is observed to trade between two distinct positions as xenon coverage increases. The applied bias between the sample and the detector remains unchanged throughout this series of scans. The offset between the peaks may be due in principle to both adsorbate-induced work function lowering and adsorbate-induced changes in the binding energy of the image potential state. 


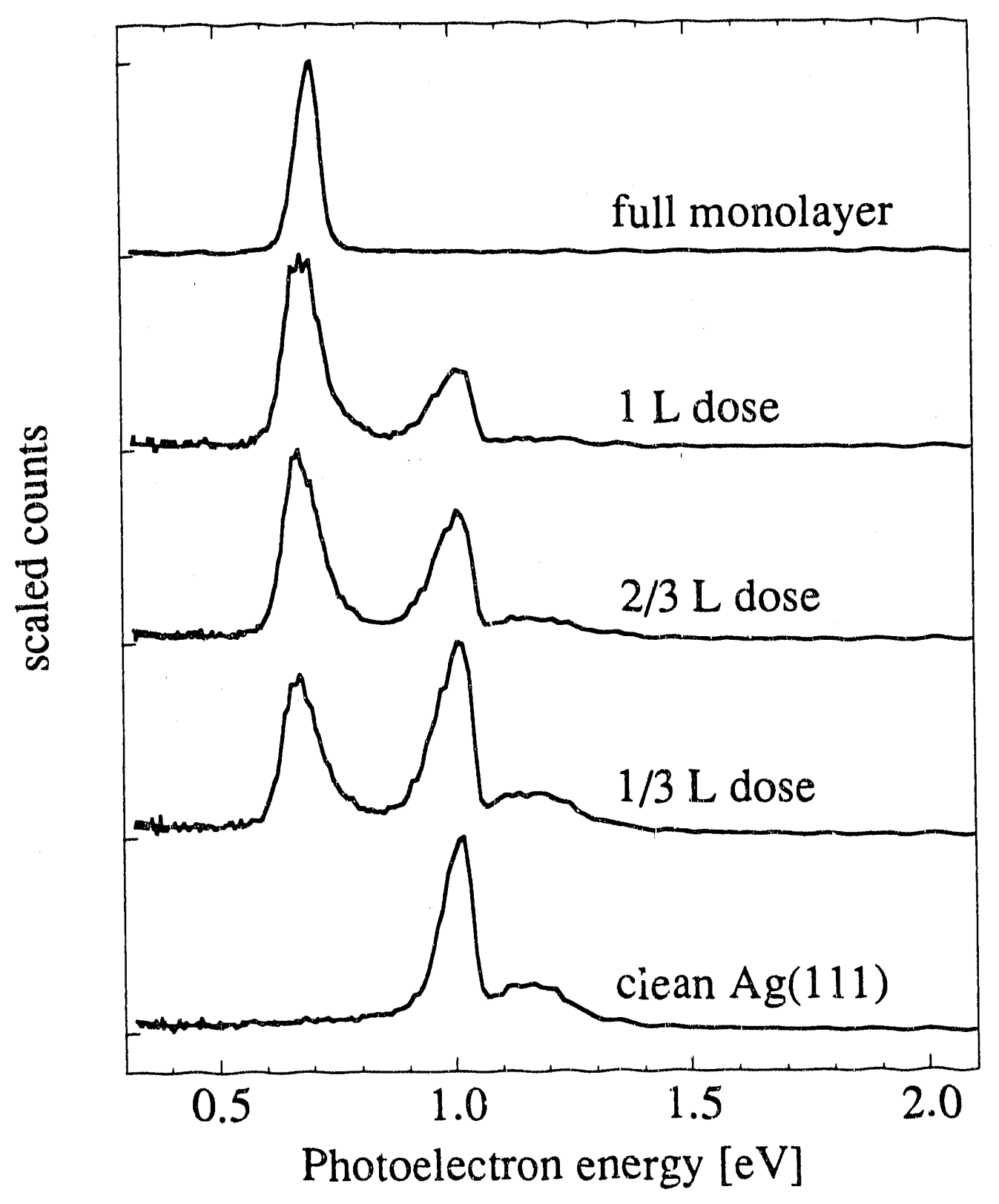

Figure 28 
original impact upon the surface. This limit of sluggish diffusion across the surface is not what one would expect, since the xenon-silver physisorption bond is quite weak, and the (111) surface of the substrate is relatively flat. Free lateral mobility of the xenon atoms, leading to nucleation of xenon islands bound by van der waals forces is the predictable behavior.

Another conclusion one is lead to upon noting the narrowness of the new feature concerns the relative spatial scales of the xenon islands and the in-plane part of the image potential state wave function. The extent of the wave function must be less than the characteristic size associated with the islands prepared in this experiment. If it were not, the image potential state would average over both clean and adsorbate-covered regions, and a broadening of the energy level should result.

In addition to any possible effects of xenon islands upon the image potential states bound to the surface, islands may also have an indirect effect upon the departing photoelectrons. Because the work function of xenon-covered regions of the surface differs from that of clean areas, contact potential differences exist between the two. The electric fields thus engendered in the microscopic region of space immediately outside the surface can conceivably be quite large due to the small length scales involved. Fields are expected to be strongest near the perimeter of each island. 
They may also be expected to scatter outgoing photoelectrons, altering both the direction and kinetic energy of the photoelectrons. If the islands were very large, the fraction of the total surface subject to the influence of these localized fields would be expected to be relatively small. Then the majority of the photoelectrons should leave the sample without suffering much scattering. If, however, the islancs were small, a large fraction of the total surface area would consist of sites adjacent to an island edge, so that the probability of a photoelectron being scattered would be relatively high, and broadening and attenuation of the sharp $n=1$ peak would result. In fact, some broadening of the peaks at intermediate coverages is apparent, so the scattering process described here may not be wholly absent. In the first approximation, though, the situation seems much closer to the large-island limit.

The difference in energy of the two peaks is potentially due to two effects. First, interaction of xenon with the surface dipole field of the metal results in a partial screening of this field due to polarization of the adsorbed atoms. This effect leads to a reduction in the work function of the sample. The consequences are diagrammed in Figure 29. This figure should be compared to figure 19, which treats contact potential effects in the adsorbate-free system. second, the xeron adlayer may shift the energy of the image potential state relative to the vacuum level. Fortunately, 
Caption for Figure 29:

In (a), the contact potential between the time-of-flight detector and the clean silver(111) crystal has been nullea by a DC bias $\left(v_{\text {init }}\right)$. In (b), physisorbed atoms or molecules have partly screened the surface dipole field of the sample. since the relative position of the bulk Fermi levels must remain fixed, but the work function of the sample is changed (by $\Delta \phi$ ), the vacuum energy in the vicinity of the sample is reduced. Accordingly, surface charges accumulate, and an electrostatic field appears between the bodies. In (C), the applied DC bias (V) has been readjusted by the amount needed to offset the work function lowering $\Delta \phi$. The electrostatic field in the vacuum is again zero. 
a)

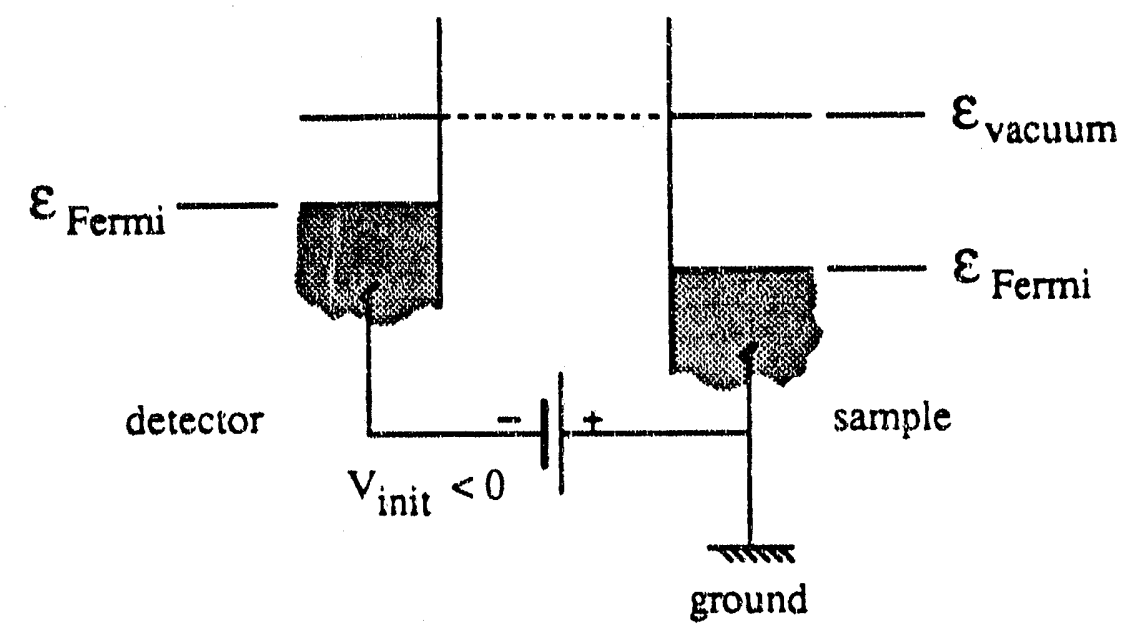

b)

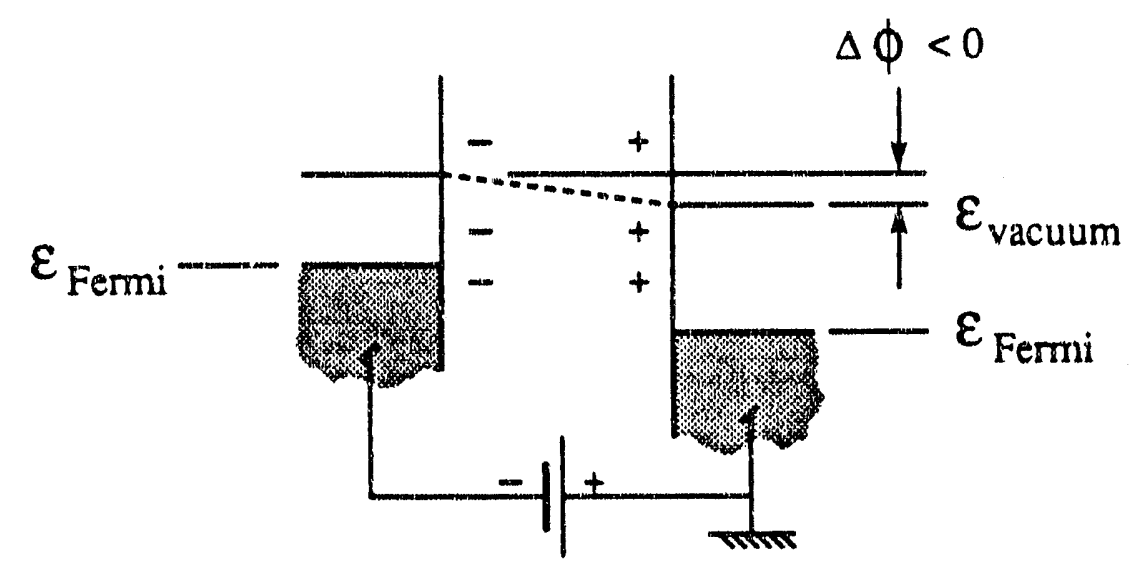

c)

Figure 29

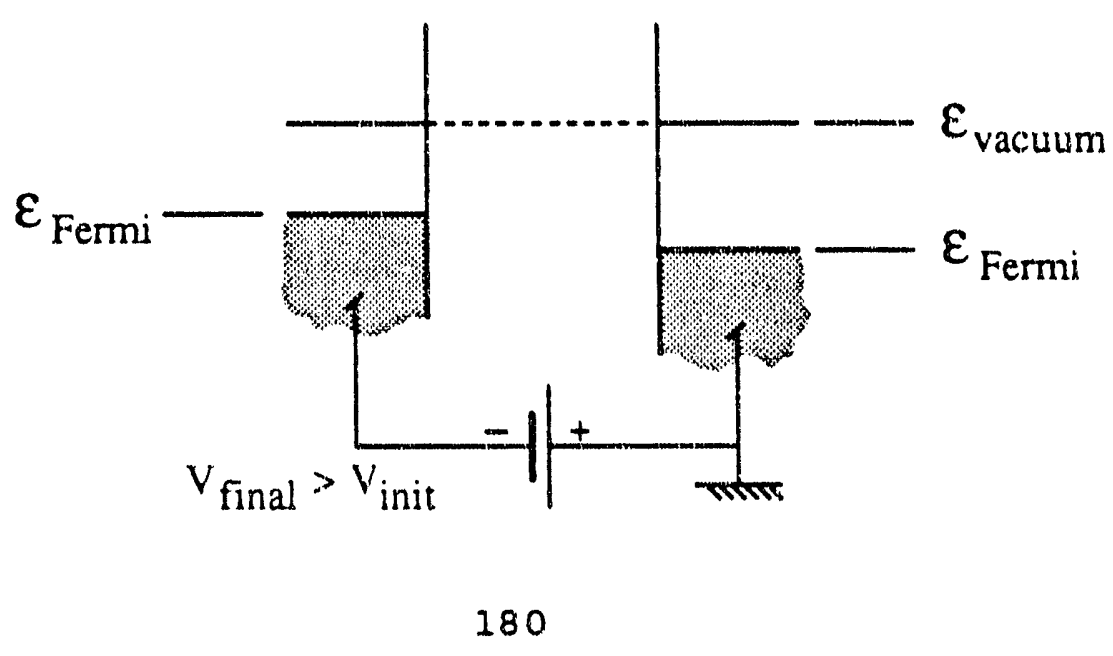


measurements may be made to separate the two effects.

since the flight time of photoelectrons is used to measure their kinetic energy, the magnitude of the adsorbateinduced work function lowering cannot accurately be read off the data in Figure 28. Instead, the work function lowering was measured independently by threshold photoemission and then the potential difference between the sample and the detector was changed to compensate for the effect. At 1 monolayer coverage, the sample's work function was found to be decreased by $0.450 \pm 0.050 \mathrm{ev}$, so the bias of the detector relative to the sample was made 0.450 volts more positive. The measured work function lowering agrees quite well with the value of $0.470 \mathrm{eV}$ reported by Behm, Brundle and wandelt. ${ }^{142}$ The adsorptioninduced change in the electrostatic field in the flight region was thus cancelled and accurate measurements of the $n=1$ image potential state binding energy in the presence of the xenon monolayer could be made.

Figuxe 30 shows the binding energy of the image potential state measured for both clean Ag(111) and the same surface covered with 1 monolayer of xenon. In the latter case, the sample temperature was $75 \mathrm{~K}$ and the ambient pressure of $\mathrm{xe}$ in the chamber was approximately $3 \times 10^{-6}$ torr (uncorrected for ion gauge response). According to the measured phase diagram of xenon on $A g(111), 138,139$ this combination of temperature and pressure results in a stable, ordered monolayer of adsorbed xenon. The significance of the data in Figure 30 is 
Caption for Figure 30 :

The dashed peak is the $n=1$ image potential state on silver (111) covered by one monolayer of xenon. The applied bias between the sample and the detector was adjusted to compensate for the adsorbate-induced change in the work function of the sample. The measured binding energy is thus accurate to within the uncertainty in the measurement of work function changes. The solidline peak, which shows the binding energy of the $\mathrm{n}=1$ image potential state on clean silver (111), is overlaid for comparison. 


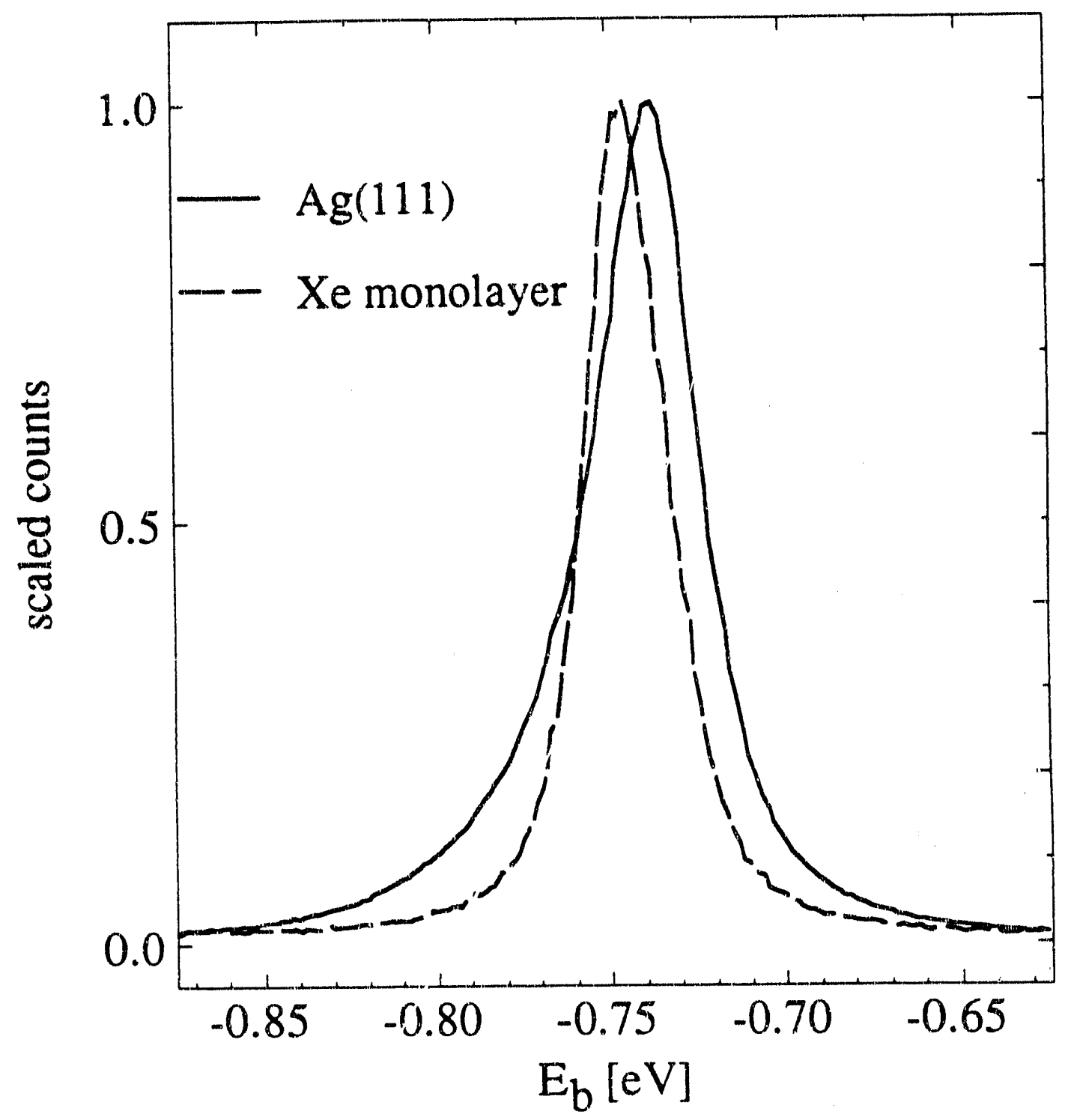

Figure 30 
principally that the binding energy of the image potential state is unchanged by the xenon monolayer within the $50 \mathrm{meV}$ uncertainty of the threshold photoemission measurements.

The apparent insensitivity of the $n=1$ binding energy to the monolayer of adsorbed xenon leads one to a pair of observations. First, dielectric screening of the image potential by the xenon layer seems to be weak or nonexistent. The dielectric constant of bulk, solid xenon has been measured as $2.09 .{ }^{144}$ The expectation value of position of the $n=1$ image potential state computed, for example, by the hydrogenic model, is on the same order as the van der waals diameter of xenon. Thus, the electronic wave function is expected to overlap spatially with the adsorbed layer. It is suggested that the effective static dielectric constant of xenon adsorbed in a monolayer on the metal surface is much closer to that of vacuum than to that of bulk xenon.

The second observation one is led to by the data in Figure 30 concerns stabilization of the electron in the image potential state by the electron affinity of of the adsorbate layer. The conduction band of bulk xenon is known tc. lie 0.5 eV below the vacuum energy. ${ }^{140,161}$ Thus, when adsorbed on $\mathrm{Ag}(111)$, it may be expected to contribute $0.5 \mathrm{eV}$ stabilization energy to the image potential state over the region of space occupied by the layer. No such additional binding energy was observed, however. Since it seems likely that the properties of xenon having to do with the outer electronic orbitals are 
quite different in the monolayer than in the bulk solid, one could suggest that the electron affinity is altered by adsorption and may be close to zero.

A third interaction distinct from the dielectric and electron affinity effects may be suggested. The layer of polarized xenon atoms which provides an electric field opposing the surface dipole and thus lowers the work function may, in the same way, present an electric field to the electron in the image potential state which would attract it to the surface. A crude estimation of the strength of this polarization field demonstrates that its effect upon the electron immediately outside the metal surface may be considerable. The induced dipole moment of 0.2 Debye per xenon atom which was calculated from the observed work function lowering by Behm, Brundie and Wandel.t 142 may be used. This dipole moment is $6.7 \times 10^{-31} \mathrm{c} \cdot \mathrm{m}$ in SI units. It is equivalent to a charge separation of +0.01 e and -0.01 e by a distance of $4 \AA$, approximately the diameter of a xenon atom. Modeling the dipole layer as a parallel plate capacitor and using a density of dipoles in the plane ${ }^{142}$ equal to that of the xenon atoms $\left(6 \times 10^{18} / \mathrm{m}^{2}\right)$, an electric field of about $10^{9} \mathrm{~V} / \mathrm{m}$ may be calculated. A field this strong should be capable of some perturbative effect upon the image potential state.

Finally, a fortuitous cancellation between dielectric screening, the electron affinity and the field due to polarization of the adsorbate cannot be ruled out. The first 
could conceivably reduce the binding energy. If the second and third were to increase it by the same amount, no net change in the measured binding energy of the image potential state would result.

Multiple reflection theory may be used to predict the energy of the image potential state after the adsorbateinduced work function shift has lowered the vacuum energy relative to the projected bulk band structure. The projected blik band gap is referenced to the Fermi energy, but the image potential state is referenced to the vacuum energy. A reduction in work function moves the image potential state down and produces a change in the coupling of the image potential state to the crystal.

The multiple reflection formalism was used to calculate the energies of the $n=1$ and $n=0$ states after allowing a drop of the vacuum energy by $0.450 \mathrm{ev}$. Otherwise, the same parameters were used as stown in Figure 26, and the same calculations were used as in the section accompanying this figure. Plots of the phase shifts resulting from the calculation are shown in Figure 31. The eigenvalues obtained are -0.690 relative to the vacuum energy for $n=1$ and $-4.274 \mathrm{eV}$ relative to the vacuum energy for $n=0$. The fact that the experimentally measured binding energy of the $n=1$ state remains unchanged within the $50 \mathrm{meV}$ uncertainty of the threshold photoemission measurement, while multiple reflection theory predicts a change of $-60 \mathrm{meV}$ is, unfortunately, barely 


\begin{abstract}
Caption for Figure 31:
Plots of crystal-induced phase shift, barrierinduced phase shift and total phase shift used in multiple reflection theory calculation for Ag(111) whose work function has been decreased by 0.450 volts. One obvious consequence of xenon adsorption on silver (111) is thus incorporated into the mutiple reflection treatment of the image potential state.
\end{abstract}



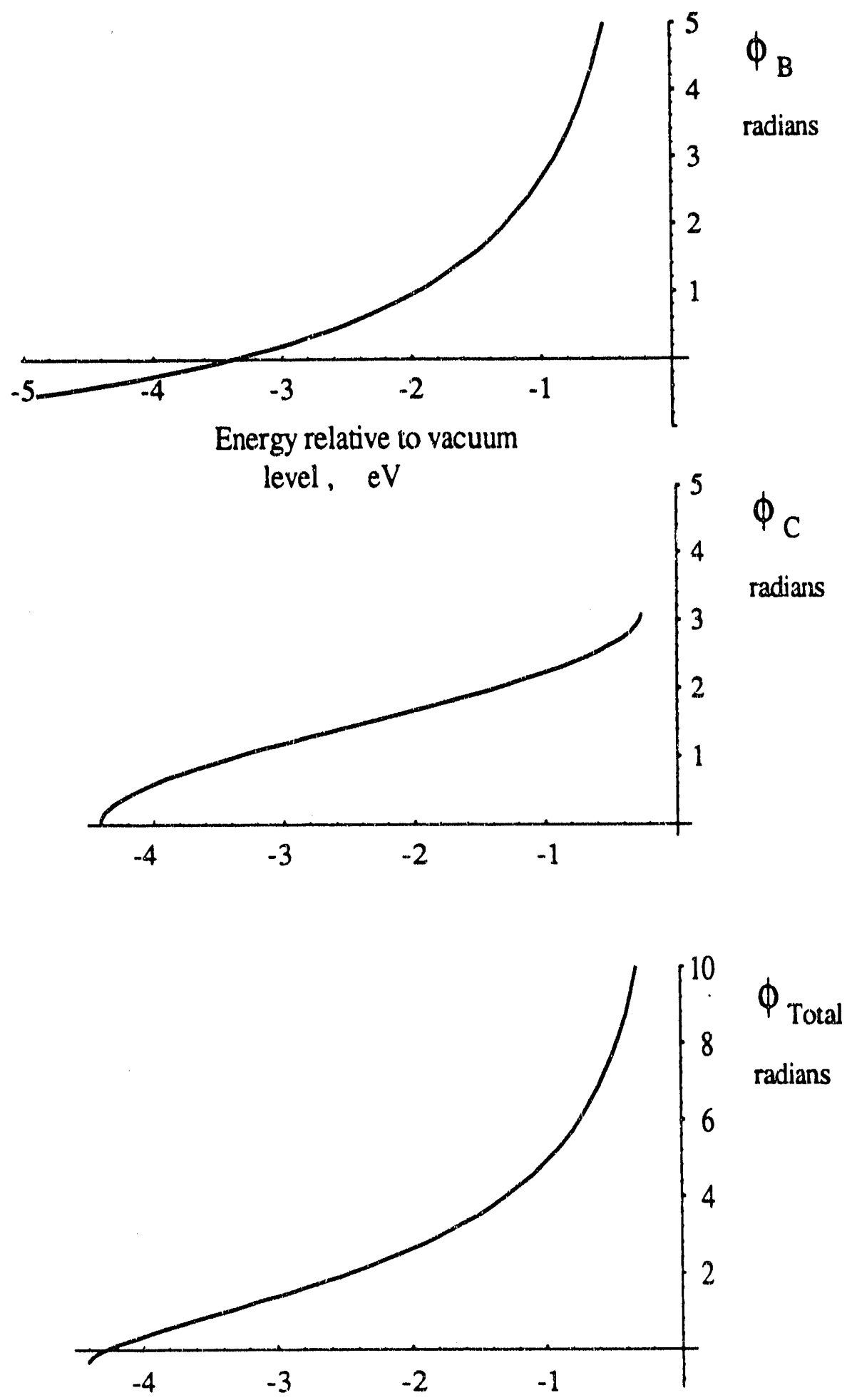

Figure 31 
conclusive. It may perhaps be taken as further evidence that fortuitous cancellation of more than one effect may be at work in this system.

It is worth noting that the peaks in Figure 30 resemble a Lorentzian form more closely than they resemble a Gaussian. Thus, it is suggested that lifetime broadening is more important than coupling to thermalized excitations of the substrate in determining the width of the measured image potential state encrgy levels. Accordingly, one may feel justified in proceeding to relate differences in peak widths directly to differences in lifetimes.

A comparison may be made between the widths of the two features in Figure 30. The widths are independent of error in the threshold photoemission measurement and are marginally greater then the energy resolution of the time-of-flight instrument around $1.2 \mathrm{eV}$ kinetic energy. Narrowing of the $n=1$ peak from $40 \mathrm{meV}$ to $25 \mathrm{meV}$ (at half height) upon deposition of the xenon monolayer is evident. This adsorption-induced narrowing appears to be the same phenomenon observed by Fischer et al. in their study of $\mathrm{Ni}(111) .96$ In that work, it was pointed out that the adsorbate may quench the surface state lying below the image potential state, and that the removal of this state as a possible decay pathway for the image potential state could increase the lifetime of the image potential state. This could produce the observed narrowing of the two-photon photoemission feature. 
Another possible explanation for the adsorption-induced narrowing involves a modification $2 f$ the image potential stare's coupling to the bulk due to the work function change associated with the adsorption of the adlayer. In this view, the work function change would drive the energy of the $n=1$ level closer to the center of the L-gap. The imaginary part of the complex wave vector describing penetration of the $\therefore$ ive function into the bulk lattice would move toward its extremal value (see Figure 6), and the penetration of the wave function tail into the lattice would decrease. Amplitude of the image potential state wave function would thus shift from the region inside the crystal to the region outside the surface. In this way, the lifetime of the state would be increased, since overlap of the wavefunction with final states associated with the crystal. would be reduced.

Figure 32 shows the experimentally detemined dispersion of the $n=1$ image potential state in the presence of an ordered xenon monolayer. The sample temperature was $75 \mathrm{k}$ and the pressure of xenon was $3 \times 10^{\circ 6}$ torr (uncorrected for jon gauge response). A parabolic dispersion relation was fit to the data, and from its slope an effective mass of $(1.00 \pm 0.05) \mathrm{m}_{\mathrm{c}}$ was obtained. This was less than the value of $(1.40 \pm 0.10) \mathrm{m}_{\mathrm{e}}$ obtained on the clean surface. One conclusion that may be drawn from this result is that the xenon layer does not show a propensity to localize the electron in the image potential state. If it did, the dispersion would become much flatter. 
Caption for Figure 32:

Image potential $(n=1)$ state energy measured relative to its value at the zone center is plotted against wave vector in the plane of the surface. sample is silver (111) covered by one monolayer of xenon. Circle symbols are used in the plot. points are obtained from a series of angle-resolved scans. The best fit parabolic dispersion relation is shown. For comparison, data points for clean sijver (111.) are shown as "+" symbols. 


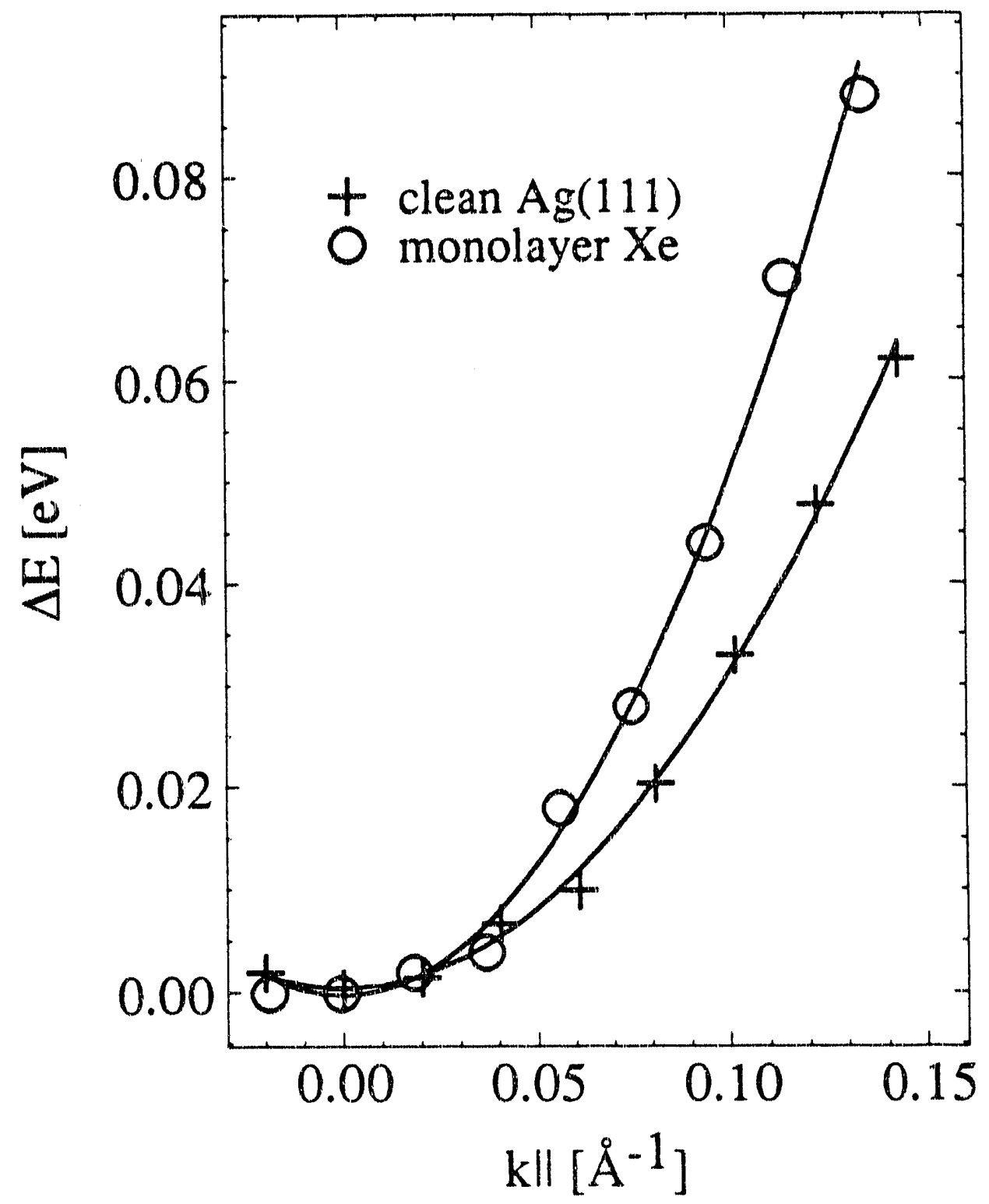

Figure 32 
It is possible that the reduction in effective mass accompanying adsorption of xenon is due to the shift in the image potential state relative to the projected bulk band gap, and particularly the upper band edge. The change in effective mass may be calculated by the method of Giesen, et al.50 Referring to the data in Table III, the energy of the upper band edge is now taken to be $-0.150 \mathrm{eV}$ relative to the vacuum energy. This change simply reflects the work function drop of $0.450 \mathrm{ev}$. For purposes of calculating the effective mass of the $n=1$ state, the lower band edge is ignored. Using equation (40), an effective mass of $1.16 \mathrm{~m}$ esults. For the sake of comparison with the calculation of Giesen, et al., the bulk band edge on the clean surface may be taken at $-0.710 \mathrm{eV}$ relative to the vacuum energy. Then, $-0.260 \mathrm{eV}$ replaces $-0.150 \mathrm{eV}$ as the energy of the upper band edge in the presence of xenon. The resulting effective mass becomes $1.20 \mathrm{~m}_{e}$. In either case, this method of estimating effective masses based on multiple reflection theory accounts for about half of the change observed experimentally. This conclusion still leaves largely open the question of the effect of the layex upon the effective mass of the image potential state. The xenon must have a more active role in determining the properties of the image potential state than merely the one mediated by the work function change due to adsorption.

3.3 Eyclohexane-covered silver (121) 
The investigation of image potential states was extencied to filins composed of molecular species. The saturated hydrocarbon cyclohexane was chosen as a representative molecule. It physisorbs on Ag(111), and the formation of ordered monolayer films on this surface has been characterized. ${ }^{145}$ The crystal structure of cyclohexane in the bulk has been determined. ${ }^{146}$

In Figure 33, the two-photon photoelectron spectrum resulting from excitation of image potential states on the monolayer-covered surface is shown. The work function shift due to adsorption was $-0.39 \mathrm{ev}$, as measured by threshold photoemission. Assuming an $n=1$ binding energy of $0.77 \mathrm{ev}$, the binding energy of the $n=2$ state is $0.30 \mathrm{eV}$. Three distinguishable peaks are at appropriate energies to be assigned as the $n=1,2$ and 3 members of the image potential series. A diffuse, small feature at slightly higher energy appears to be composed of overlapping, higher members of the series. This result constitutes the first observation of members of the image potential series higher than $n=2$ on any metal surface. It is not clear why this dramatic result was obtained when cyclohexane was adsorbed, but not when xenon was adsorbed. The work function of clean Ag(111) is too high for the lasex to efficiently excite the entire series in the absence of a work-function-lowering adsorbate.

Shown in Figure 34 are the dispersions of the $n=1$ and 2 states. The effective masses of both the $n=1$ and $n=2$ states 
Caption for Figure 33:

Kinetic energy distribution of two-photon photoelectrons emitted normal to silver (111) surface when covered by one monolayer of cyclohexane. At least three members of the image potential series are resolvable and are labeled. 


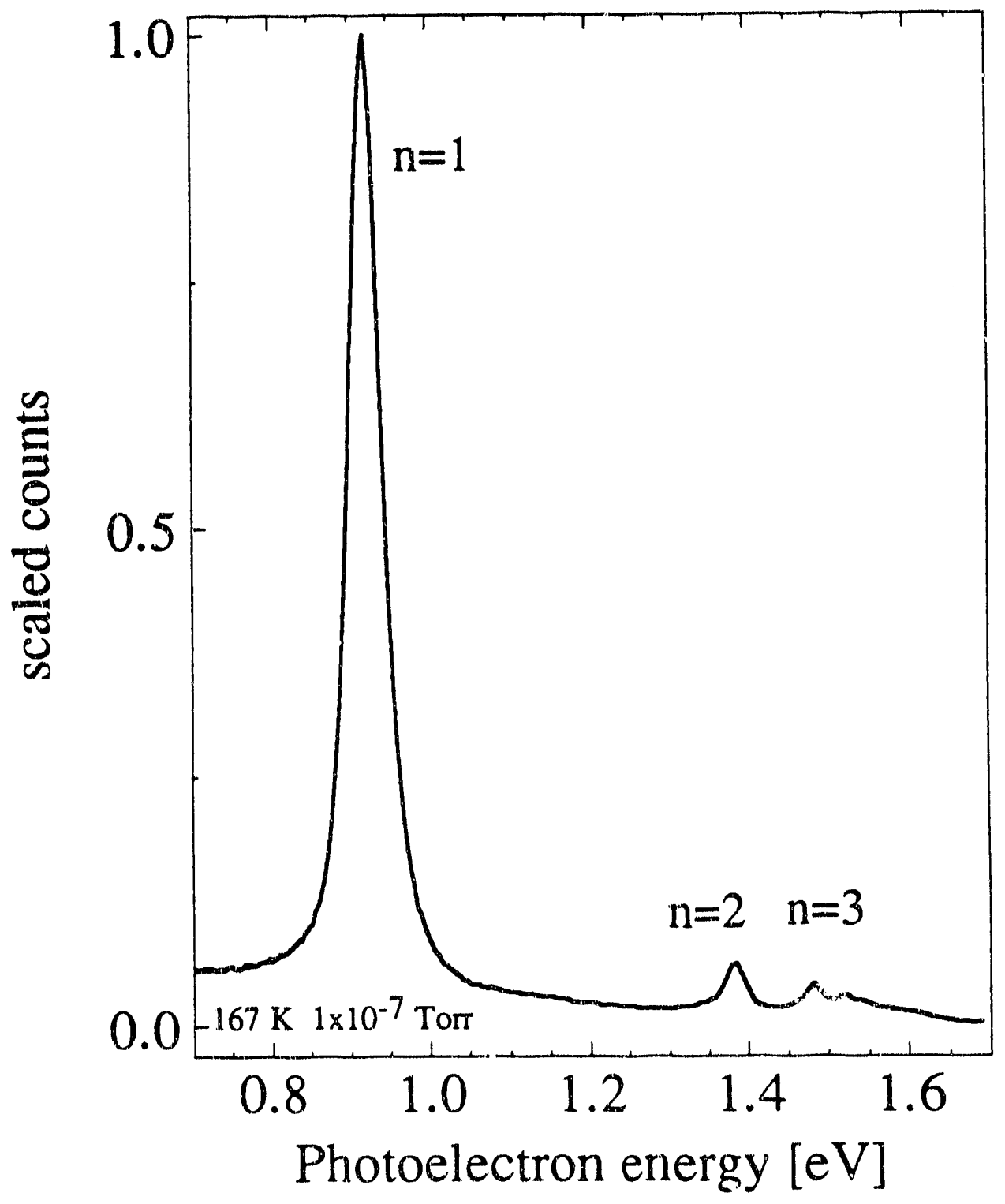

Figure 33 
Caption for Figure 34 :

At the top, the experimentally determined dispersion relation of the $n=1$ image potential state on silver(III) covered by a monolayer of cyclohexane is shown. At the bottom, the dispersion relation of the $n=2$ image potential state under the same conditions is shown. The parabola best fitting the data points is indicated, along with the corresponding effective mass. 


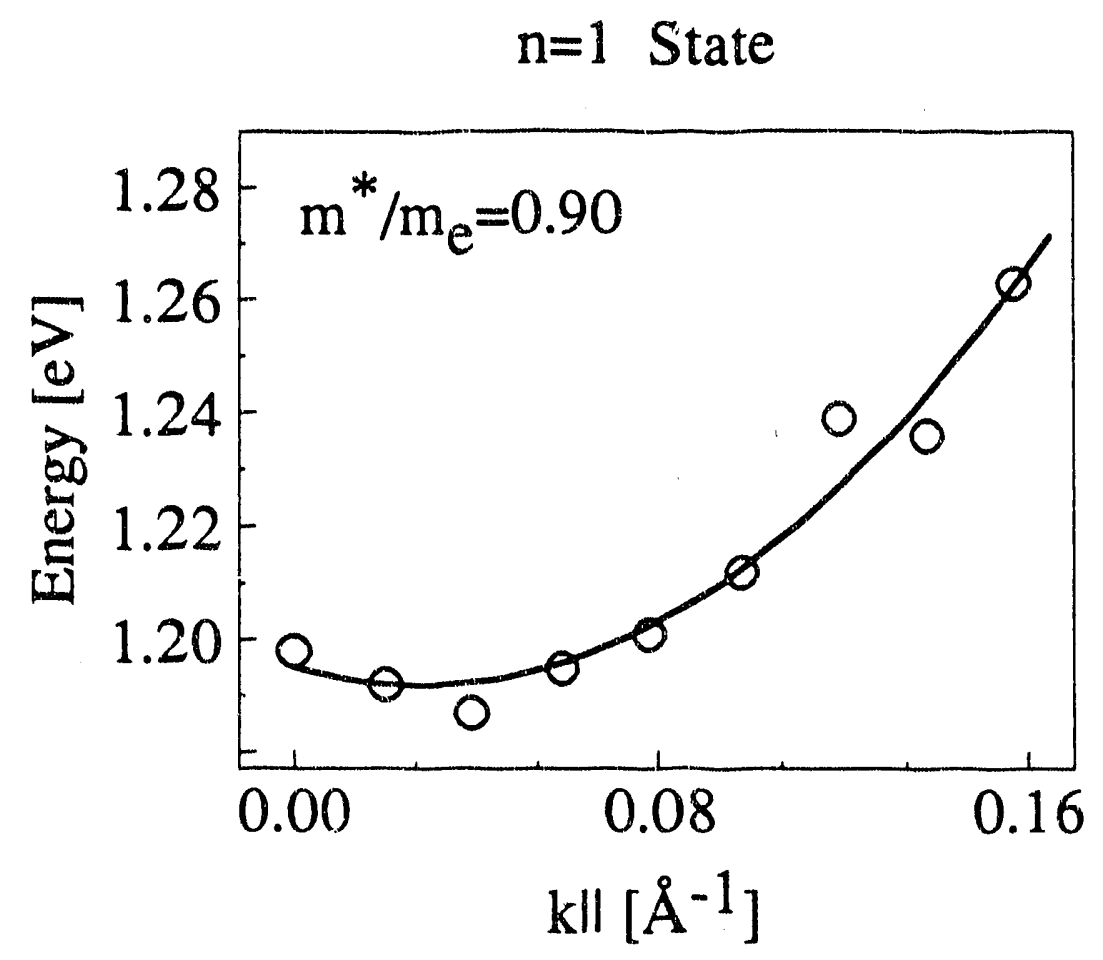

$n=2$ State

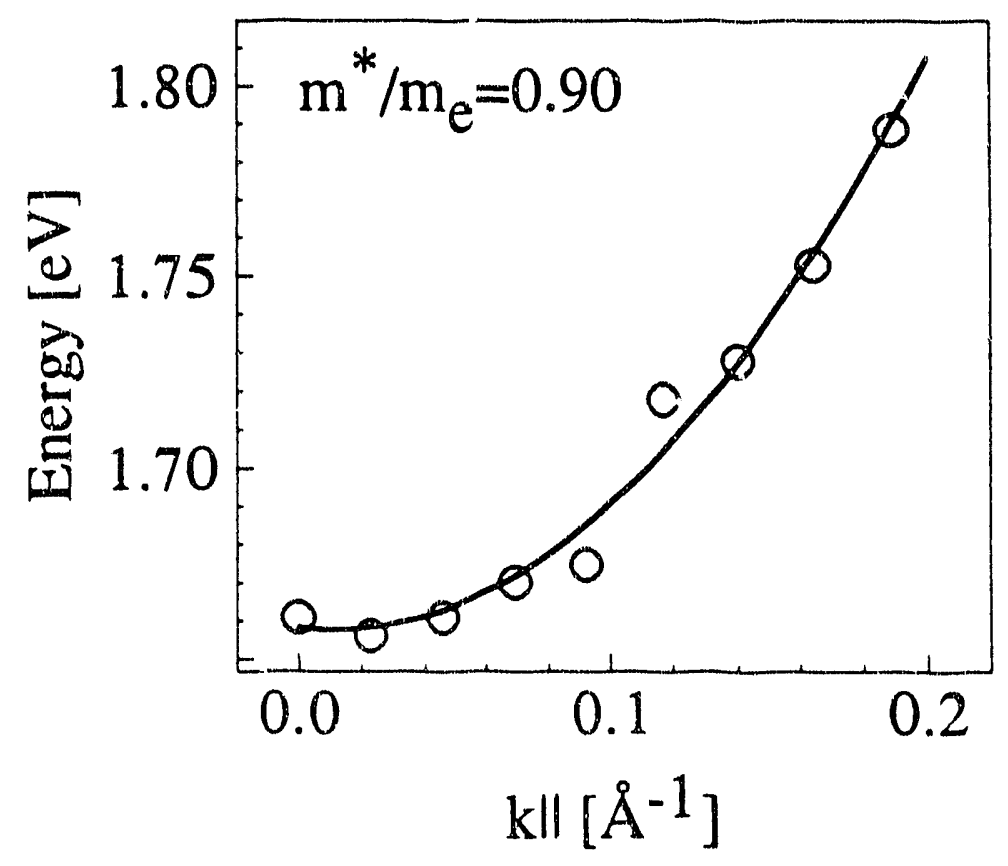

Figure 34 
are $(0.90 \pm 0.10) \cdot m_{e}$. As was the case with xenon, much of the reduction in the effective mass can be attributed to the modified coupling of the image potential state with the upper projected bulk bant edge. No evidence is apparent for localization of the image potential state by adsorbate molecules, but other interactions with the adsorbate cannot necessarily be ruled out.

Figure 35 contains dramatic evidence of the image potential state's sensitivity to the progressive growth of cyclohexane multilayers. At zexo coverage, the $n=1$ image potential state is prominent and a small feature due to nonresonant two-photon-photoemission from the occupied surface state appears. Adsorption of the first layer of cyclohexane quenches the occupied surface state and shifts the $n=1$ feature to lower energy. Based on the thresiold photoemission measurements, all of this shift in the kinetic energy of the photoelectrons can be attributed to the adsorption-induced drop in work function. As was the case for xenon, no evidence is present for dielectric screening or electron affinity stabilization in the first monolayer. The work function change saturated with the completion of the first monolayer, however, and as subsequent layers were grown, pronounced changes in the $n=1$ state appeared. As Figure 35 shows, the peak zvolves through five distinct energies in a stepwise fashion. Intensity in the peaks trades toward lower binding energies as the mean thickness of the adsorbate film 
Caption for Figure 35:

Langmuir dosing of cyclohexane on silver (111). Features attributable to $n=1$ image potential state are prominent. A small peak due to nonresonant excitation of the occupied surface state is apparent at higher energy when the surface is nearly clean. The complicated evolution of the $\mathrm{n}=1$ feature with increasing adlayer coverage is evident. 


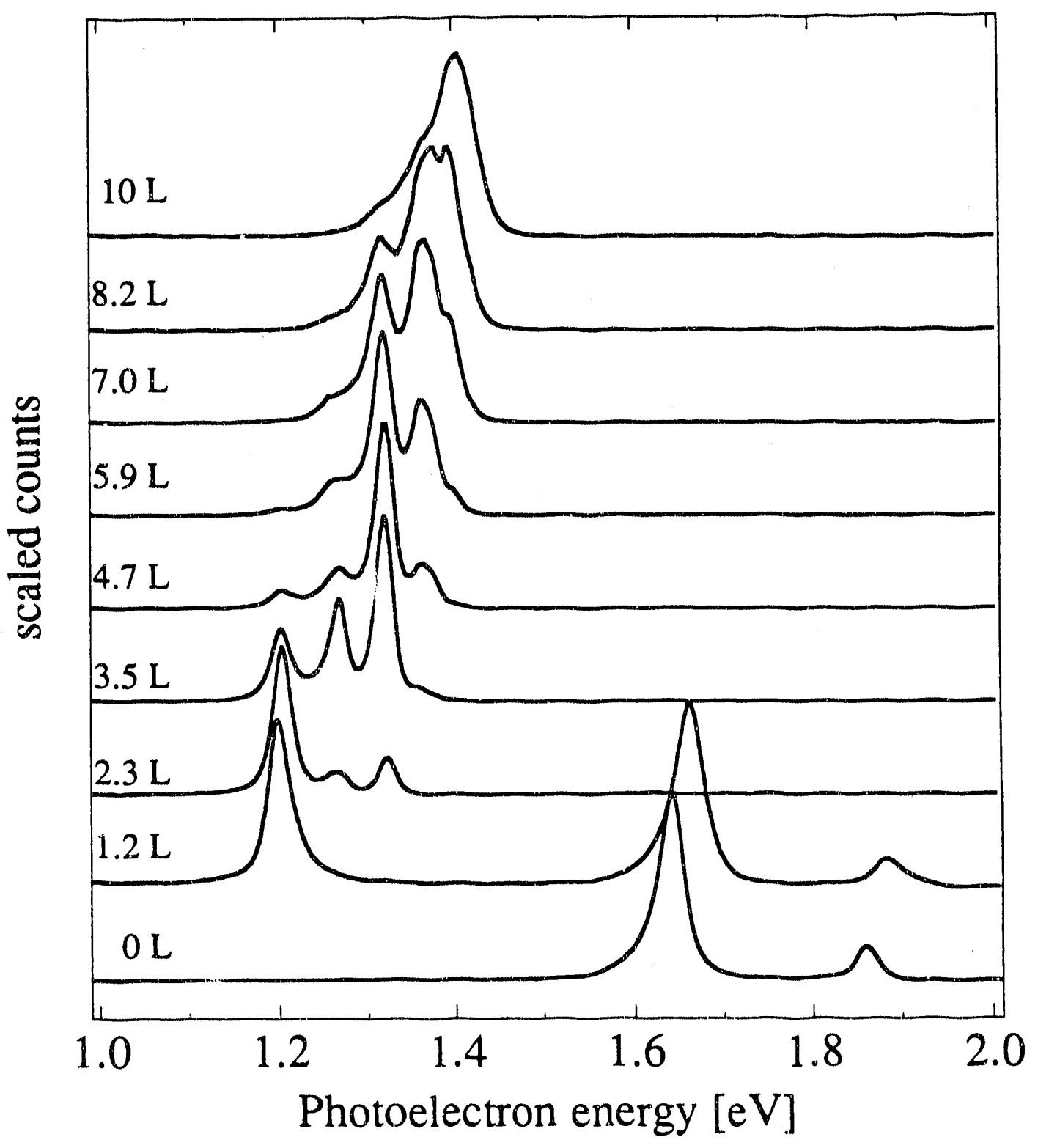

Figure 35 
increases. The binding energy of the $n=1$ state is determined by the thickness of the adsorbate layer it samples, and, since the layer thickness increases in discrete intervals determined by the molecular dimensions, the observed set of distinct binding eivergies is generated.

The thickness increment added by each successive layer may be inferred rrom the existing experimental information on the structure of cyclohexane multilayers on $\mathrm{Ag}(111)$ and the crystal structure of bulk cyclohexane. In an $x$-ray diffraction study, Kahn, Fourme, Andre and Renaud ${ }^{146}$ have determined that, below about $180 \mathrm{~K}$, cyclohexane grows a monoclinic unit cell with dimensions $a=11.23 \AA$, $b=6.44 \AA$, $C=8.20 \AA$ and $\beta=108.83^{\circ}$. The number of molecules per unit cell is four. If, for the moment, one were to regard the molecules (crudely) as discs, they would be stacked parallel in the crystal with their flat surfaces nearly normal to the c axis. One could then say that, in the bulk, each layer of cyclohexane molecules is about $4 \mathrm{~A}$ thick, based on the lattice constant $c$.

For multilayers of cyclohexane on Ag(111), Firment and Somorjai ${ }^{145}$ observed a $(9 \times 9)$ surface structure using LEED. cyclohexane molecules formed a hexagonal surface mesh which was essentially identical to the unreconstructed $a-b$ plane in the bulk cycloherane crystal. While the LEED experiment cannot be expected to to give the spacing between adsorbate layers, it seems plausible that this spacing must be close to 
4 A based on the close similarity between the surface structure of the adsorbed multilayer and the arrangement of molecules in the bulk crystal.

The principal question to be answered concerning the evolution of the $n=1$ state with successive growth of cyclohexane layers is whether the physical mechanism for the shift is dielectric screening or an electron affinity interaction with the film. A clue may be taken from consideration of the spatial scales involved. The expectation value for the $n=1$ state's position is about $3 \AA$ outside the surface. This is also approximately the thickness of a single layer of cyclohexane. The image potential state would be effectively buried after completion of the second layer, and, if only dielectric interactions were at work, the eigenvalue would be little affected by the growth of subsequent layers.

on the other hand, an electron affinity interaction may predominate. The electron affinity of cyclohexane in the gas phase has been measured by electron transmission spectroscopy as $-4.11 \mathrm{eV} \cdot{ }^{147}$ In the condensed phase, polarization interactions should make the electron affinity somewhat less negative, but it is still likely that an electron forced to exist in bulk cyclohexane would lie at an energy higher than the vacuum level. The cyclohexane film could thus modify the image potential by adding to it a repulsive barrier whose width would be the thickness of the film. Accordingly, the eigenvalue would shift to higher energies as the layer grew, 
which is just the result observed experimentally. While this effect should saturate as the layer thickness becomes large, the saturation may occur more slowly than for the dielectric interaction, since the probability density of the image potential state would not be buried at the early stages of film growth, but would be forced out from the surface as the film grew.

Magnification of the third scan in Figure 35 allows the relatively small structure around $1.6 \mathrm{eV}$ to be examined. This appeurs to be composed of 3 or 4 resolved peaks which are assigned as nigher members of the image potential state series. These are shown in Figure 36. They are the same fentures evident in Figure 33. It is significant that these peaks are unaffected by the growth of cyclohexane multilayers, while the $n=1$ peak clearly is split into 3 features assignable to 1-, 2- and 3-layer-deep patches.

At this point, firm conclusions cannot be drawn on the exact physical mechanism behind the observed difference in the influence of multilayer growth lipon the $n=1$ and $n=2$ image potential states. It may be suggested that a difference in the degree of dielectric screening of the probability density of the $n=1$ and $n=2$ states is the dominant effect. The $n=2$ state, with an expectation value of position equal to about 13 $A$, is further from the surface, so this state may be less sensitive to growth of the first few molecular layers. on the other hand, if the electron affinity of the adlayer were 
Caption for Figure 36:

Enlargement of the two-photon photoelectron kinetic energy distribution obtained with approximately 2.3 Langmuirs cyclohexane exposure. Higher members of the image potential series are small but distinguishable. 


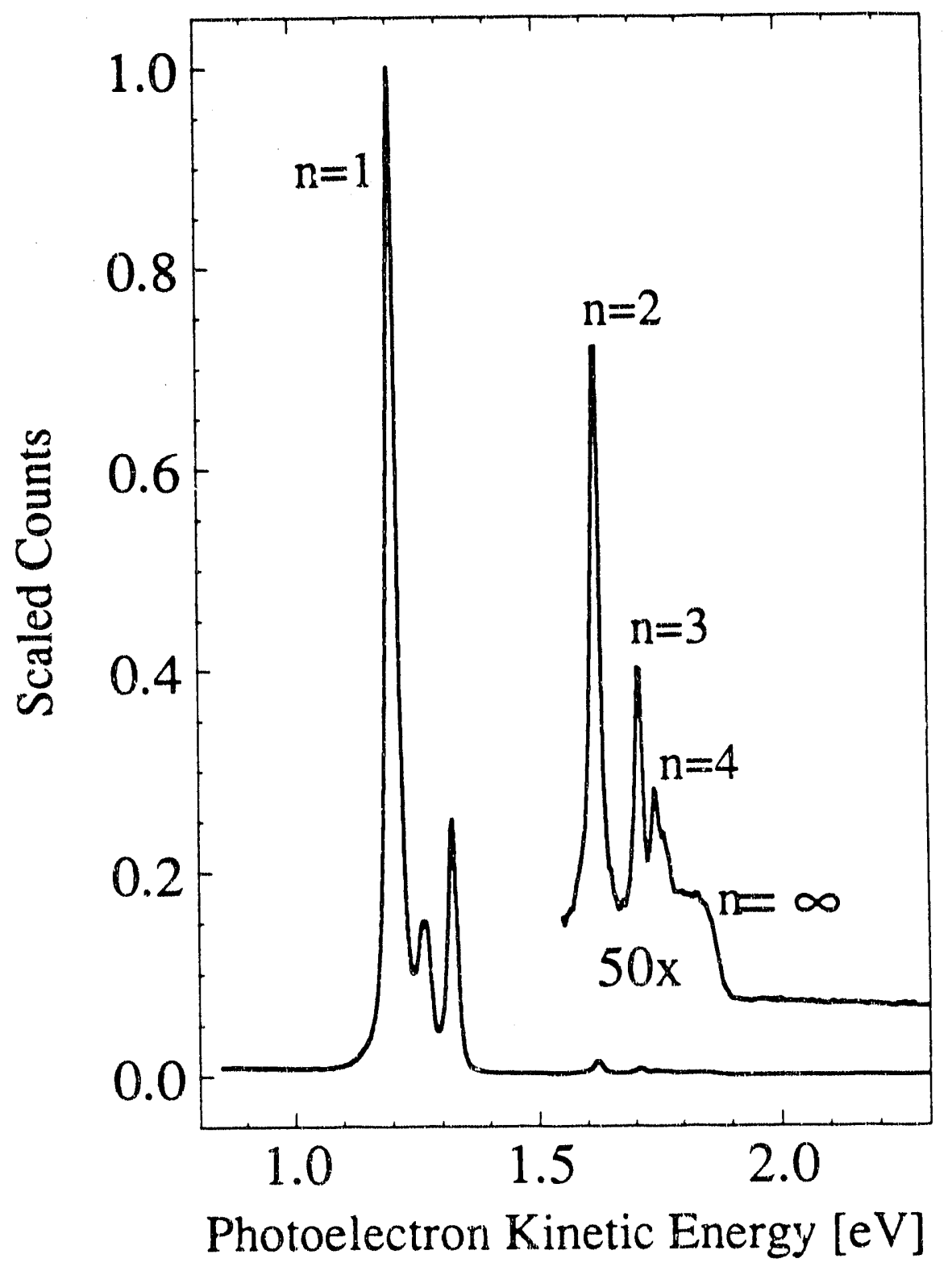

Figure 36 
negative and significantly different from zero, the repulsive barrier it would provide could shift the $n=1$ and $n=2$ eigenvalues by different amounts. Partial exclusion of amplitude from the 2- and 3-layer-deep patches by a negative electron affinity might not affect the eigenvalue strongly, however, since much of the image potential state's amplitude is already outside this region. In essence, exclusion of the relatively small fraction of the $n=2$ wave function which lies in the region of space overrun by the bilayer and trilayer should alter the observable characteristics of the $n=2$ state, particularly the eigenvalue, only weakly.

Finally, the polarization field existing in the first monolayer of adsorbed molecules is likely to have a significant effect on the overall form of the electronic potential existing outside the surface. Quantitative calculations exploring the relative impact of all three of these effects upon the wave functions and eigenvalues are clearly necessary to reach a comprehensive understanding of the physics at work in this system. 
In this work, image potential states have been shown to probe certain properties of metal-dielectric interfaces with great sensitivity. Their response to the range of microenvironments provided by patches of molecular adsorbate layers with varying thickness is particularly remarkable. In light of this result, the apparent insensitivity of image potential states to the deposition of the first layer of adsorbate species on the surface is surprising and mysterious. It is apparent that the direct proximity of the metal surface strongly modifies either the energy level stxucture of the adsorbate, and thus its electron affinity, or the dielectric properties of the adsorbate, or perhaps both.

The versatility of simple multiple reflection theory in predicting the properties of image potential ststes on metal surfaces was demonstrated in this work. Merely by incorporating adsorption-induced work function shifts into the calculations, qualitatively correct results were obtained for both binding energies and effective masses of the inage potential states in the metal-dielectric system. The lack of more exact numerical agreement of the data with the calculated results does imply, however, that perhaps not all of the relevant physics is captured by this model. The particular strength of multiple reflection theory is the simple, yet somewhat elegant way in which it takes account of the effect 
of the lattice upon the image potential state. This advantage is especially important on surfaces besides Ag(111), where the crystal-induced phase shift may not be so conveniently close to $\pi$, and models of the hydrogenic type may not provide such a serendipitously accurate description of the image potential state.

The generalization of multiple reflection theory to treat image potential states in the presence of adsorbed layers is a worthwhile goal for the future. Incorporation of both dielectric screening and electron affinity interactions should be possible, although this would greatly complicate the evaluation of the barrier-induced phase shift. Numerical integration to determine the phase shift would be a versatile and pragmatic approach to the problem.

In further modeling of adlayer effects upon the image potential state, modification of the electronic properties of the adsorbate by the metal substrate should not be overlooked. The observed insensitivity of image potential states to one monolayer of adsorbate confirms this point. Dielectriz and electron affinity interactions of the electron with the adsorbate are only worth discussing if the orbital structure of the adsorbed species is well defined.

In the immediate future, the experimental work on physisorbed materials could be extended profitably by focussing on other noble gases and multilayers of noble gases. A survey of the effects of monolayers of various noble gases 
upon the image potential state is appealing because these are the simplest and perhaps the best characterized of all physisorbed systems. The phase diagrams and structures of the layers are known, and the atoms do not have troublesome optical absorptions in the near UV. Multilayers of xenon and other noble gases would provide valuable information on the effects of dielectric screening and electron affinity of the growing adlayer upon the image potential states. In particular, the electron affinity of noble gases in the

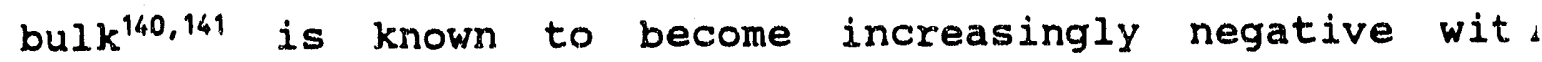
decreasing atomic weight. This trend in electron affinity may be manifested in the dependence of image potential state binding energy upon the thickness of the noble gas layer. Angle-resolved two-photon photoemission studies of multilayers of argon or krypton pose a truly formidable technical challenge, since they would require the sample to be cooled to about $20 \mathrm{~K}$. Nonetheless, a wide range of obviously feasible experiments would become possible upon successfully solving this problem.

synchronously pumped lasers and time-of-flight photoelectron energy analys is have been proven to complement one another admirably in two-photon photoelectron spectroscopy. In this work, the combination of superior energy resolution, negligible space charge broadening and high average countrate was nearly optimal. It is hoped that this experimental technique may also be extended profitably to two- 
photon photoemission studies of chemisorbed systems, adsorbate negative ions, metal-semiconductor interfaces, and perhaps also vibrationally inelastic two-photon photoemission of image potential states in systems incorporating molecular adsorbates. 


\section{References}

1. D. J. Griffiths, Introduction to Electrodynamics, (Prentice-Hall, 1981).

2. M. W. Cole and M. H. Cohen, Phys. Rev. Lett., 23, 1238, (1969).

3. M. Karplus and R. N. Porter, Atoms and Molecules, (Benjamin/Cummings, 1970).

4. P. W. Atkins, Molecular Quantum Mechanics, (Oxford, 1983).

5. A. Zangwill, Physics at surfaces, (Cambridge, 1988).

6. E. G. McRae, Sur. Sci., 25, 491, (1971).

7. E. G. McRae, J. Vac. Sci. Tech., 16(2), 654, (1979).

8. E. G. MCRae, Rev. Mod. Phys., 51, 541, (1979).

9. E. G. MCRae and M. L. Kane, Sur. Sci., 108, 435, (1981).

10. P. M. Echenique and J. B. Pendry, J. Phys. C: Sol. St. Phys., 11, 2065, (1978).

11. D. Straub and F. J. Himpsel, Phys. Rev. Lett., 52, 1922, (1984).

12. B. Reihl and R. R. Schlittler, Phys. Rev. B, 29, 2267, (1984).

13. P. J. Feibelman and D. E. Eastman, Phys. Rev. B, 10, 4932, (1974).

14. B. Reihl, K. H. Frank and R. R. Schlittler, Phys. Rev. B, 30, 7328, (1984).

15. V. Dose, W. Altmann, A. Goldmann, U. Kolac and J. Rogozik, Phys. Rev. Lett., 52, 1919, (1984).

16. P. D. Johnson and N. V. Smith, Phys. Rev. B, 27, 2527, (1983).

17. D. P. Woodruff, N. V. Smith, P. D. Johnson and W. A. Royer, Phys. Rev. B, 26, 2943, (1982).

18. G. Denninger, V. Dose and H. Scheidt, Appl. Phys., 18, 375, (1979). 
19. D. A. Wesner, P. D. Johnson and N. V. Smith, Phys. Rev. B, 30, 503, (1984).

20. N. V. Smith, Phys. Rev. B, 32, 3549, (1985).

21. E. W. Plummer and W. Eberhardt, Adv. Chem. Phys., 49, $533,(1982)$.

22. R. F. Garrett, N. v. Smith, Phys. Rev. B, 33, 3740, (1986).

23. C. T. Chen, N. V. Smith, Phys. Rev. B, 35, 5407, (1987).

24. S. Papadia, M. Persson and L. A. Salmi, Phys. Rev. B, 1, 10237, (1990).

25. N. V. Smith, Rep. Prog. Phys., 51, 1227, (1988).

26. P. M. Echenique and J. B. Pendry, Prog. in Sur. Sci., 32, 111, (1.990).

27. S. L. Hulbert, P. D. Johnson, N. G. Stoffel and N. V. Smith, Phys. Rev. B, 32, 3451, (1985).

28. S. L. Hulbert, P. D. Johnson, N. G. Stoffel, W. A. Royer and N. V. Smith, Phys. Rev. B, 31, 6815, (1985).

29. D. P. Woodruff, S. L. Hulbert, P. D. Johnson and N. V. Smith, Phys. Rev. B, 31, 4046, (1985).

30. R. A. Bartynski, T. Gustafsson and P. Soven, Phys. Rev. B, 31, 4745, (1985).

31. A. Goldmann, V. Dose and G. Borstel, Phys. Rev. B, 32, 1971 , (1985).

32. N. Memmel, G.' Rangelov, E. Bertel and V. Dose, Phys. Rev. B, 43,6938, (1991).

33. S. A. Iindgren and I. Wallden, Phys. Rev. Lett., 59, $3003,(1987)$.

34. S. A. Lindgen and L. Wallden, Phys. Rev. B, 38, 3060, (1988).

35. K. Giesen, F. Hage, F. J. Himpsel, H. J. Riess and W. Steinmann, Phys. Rev. B, 35, 971, (1987).

36. S. Schlupper, N. Fischer, w. Steinmann, R. Schneider and E. Bertel, Phys. Rev. B, 12, 9403, (1990). 
37. N. Fischer, S. Schuppler, R. Fischer, T. Fauster and $W$. steinmann, Phys. Rev,. B, 43, 14722, (1991).

38. G. D. Kubiak, J. Vac. Sci. Tech. A, 5(4), 731, (1987).

39. G. D. Kubiak, Sur. Sci., 201, L475, (1988).

40. A. V. Hamza and G. D. Kubiak, J. Vac. Sci. Tech. A, 8(3), 2687 , (1990).

41. A. -W. Maue, Z. Phys., 94, 717, (1935).

42. E. T. Goodwin, Proc. Camb. Phil. Soc., 35, 205, (1939).

43. B.Feuerbacher, ed., Photoemission and the Electronic Properties of surfaces, (Wiley, 1978), p.193.

44. F. Forstmann, $z$, phys., 235, 69, (1970).

45. N. W. Ashcroft and N. D. Mermin, solid state Physics, (Saunders Coljoge, 1976).

46. V. Heine, Sur. Sci., 2, 1, (1964).

47. M. H. Cohen and V. Heine, Adv. Phys., 7, 395, (1958).

48. N. Garcia and J. Solana, Sur. Sci., 36, 262, (1973).

49. Z. Lenac, M. Sunjic, H. Conrad and M. E. Kordesch, Phys. Rev. B, 36, 9500, (1987).

50. K. Giesen, F. Hage, F. J. Himpsel, H. J. Riess, W. steinmann and N. V. Smith, Phys. Rev. B, 35, 975, (1987).

51. F. M. Echenique, J. Phys. C: Sol. St. Phys., 18, L1133, (1985).

52. P. M. Echenique, F. Flores and F. Sols, Phys. Rev. Lett, $55,2348,(1985)$.

53. J. Baussells and P. M. Echenique, Phys. Rev. B, 33, 1471, (1986).

54. P. I. de Andres, P. M. Echenique and F. Flores, Phys. Rev. B, 39, 10356, (1989)。

55. P. L. de Andres, P. M. Echenique and F. Flores, Phys. Rev. B, 35, 4529, (1987).

56. X. Y. Zheng, R. H. Ritchie and J. R. Manson, Phys. Rev. B, 39, 13510, (1989). 
57. X. Y. zheng and R. H. Ritchie, Phys. Rev. B, 43, 4002, (1991).

58. S. L. Hulbert, P. D. Johnson, M. Weinert and R. F. Gacrett, Phys. Rev. B, 33, 760, (1986).

59. P. Gies, J. Phys. C: Sol. St. Phys., 19, L209, (1986).

60. S. Ossicini and C. M. Bertoni, J. Vac. Sci. Tech. A, $5(4), 727,(1987)$.

61. A. Kiejna, Phys. Rev. B, 43, 14695, (1991).

62. M. Weinert, S. L. Hulbert and P. D. Johnson, Phys. Rev. Lett, 55, 2055, (1985).

63. C. dew. van Siclen, Phys. Rev. B, 41, 8175, (1990).

64. N. Garcia, B. Reihl, K. H. Frank and A.R. Williams, Phys. Rev. Lett, 54, 591, (1985).

65. K. Giesen, F. Hage, F. J. Himpsel, H. J. Riess and W. Steinmann, Phys. Rev. B, 33, 5241, (1986).

66. D. Straub and F. J. Himpsel, Phys. Rev B, 33, 2256, (1986).

67. R. Shakeshaft and L. Spruch, Phys. Rev. A, 31, 1535, (1985).

68. A. A. Grinberg, Phys. Rev. B, 32, 8187, (1985).

69. G. R. Freeman, Kinetics of Nonhomogeneous Processes, (Wiley, 1987).

70. M. W. Cole, Phys. Rev. B, 3, 4418, (1971)

71. J. Unguris, L. W. Bruch, E. R. Moog and M. B. Webb, Sur. Sci., 87, 415, (1979).

72. V. Dose, J. Phys. Chem., 88, 1681, (1984).

73. V. Dose, Sur. Sci. Rep., 5, 337, (1985).

74. W. Altmann, V. Dose and A. Goldmann, Z. Phys. B., 65, $171,(1986)$.

75. A. Otto, K. H. Frank and B. Reihl, Sur. Sci., 163, 140, (1985).

76. A. Otto and B. Reihl, Sur. Sci,, 178, 635, (1986). 
77. K. H. Frank, F. Yannoulis, R. Dudde and E. E. Koch, J. Chem. Phys., 89, 7569, (1988).

78. S. Yang, K. Garrison and. R. A. Bartynski, Phys. Rev. B, 43, 2025, (1991).

79. K. H. Frank, R. Dudde and E.E. Koch, Phem. Phys. Lett, $132,83,(1986)$.

80. A. Otto, K. H. Frank and B. Reihl, Sur. Sci, 162, 891, (1985).

81. B. Reihl, R. R. Schlittler and H. Neff, Phy. Rev. Lett, 52, 1826, (1984).

82. B. Reihl, Sur. Sci., 162, 1, (1985).

83. W. Jacob., E. Bertel and V. Dose, Phys. Rev. B, 35, 5910, (1987).

84. G. Borstel, G. Thorner, M. Donath, V. Dose and A. Goldmann, Sol. St. Comm., 55, 469, (1985).

85. F. J. Himpsel, Adv. Phys., 32, 1, (1983).

86. K. Giesen, F. Hage, H. J. Riess, W. Steinmann, R. Haight, R. Beigang, R. Dreyfus, Ph. Avouris and F. J. Himpsel, Physica Scripta, 35, 578, (1987).

87. W. Steinmann, Appl. Phys. A, 49, 365, (1989).

88. J. Bokor and R. Haight, Angle-Resolved Photoemission, (S. Kevan, ed., to be published).

89. J. Bokor, Science, 246, 1130, (1989).

90. K. Giesen, F. Hage, F. J. Himpsel, H. J. Riess and W. Steinmann, Phys. Rev. Lett., 55, 300, (1985).

91. R. W. Schoenlein, J. G. Fujimoto, G. L. Eesley and T. W. Capehart, Phys. Rev. B, 43, 4688, (1991).

92. H. W. Rudolf, W. Steinmann, Sur. Sci., 93, 107, (1980).

93. D. Rieger, T. Wegehaupt and w. Steinmann, Phys. Rev. Lett. , 58, 1135, (1987).

94. N. Fischer, S. Schuppler, Th. Fauster and W. Steinmann, Phys. Rev. B, 42, 9717, (1990).

95. H. W. Rudolf, D. Rieger and w. Steinmann, sol. st. Comm., $34,427,(1980)$. 
96. T. Wegehaupt, D. Rieger and W. steinmann, Phys. Rev. B, $37,10086,(1988)$.

97. R. W. Schoenlein, J. G. Fujimoto, G. L. Eesley and W. Capehart, Ultrafast Phenomena 6, (Springer Verlag, 1988).

98. R. W. Schoenlein, J. G. Fujimoto, G. I. Eesley and T. W. Capehart, Phys. Rev. Lett., 61, 2596, (1988).

99. R. T. Williams, T. R. Royt, J. C. Rife, J. P. Long and M. N. Kabler, J. Vac. Sci. Tech., 21(2), 509, (1982).

100. R. Haight, J. Bokor, J. Stark, R. H. Storz, R. R. Freeman and P. H. Bucksbaum, Phys. Rev. Lett., 54, 1302, (1985).

101. J. Bokor, R. Haight, R. H. Storz, J. Stark, R. R. Freeman and P. H. Bucksbaum, Phys. Rev. B, 32, 3669, (1985).

102. R. Haight, J. Bokor, Phys. Rev. Lett., 56, 2846, (1986).

103. R. Haight, J. A. Silberman, Phys. Rev. Lett., 62, 815, (1989).

104. J. Bokor, R. Storz, R. R. Freeman and P H. Bucksbaum, Phys. Rev. Lett, 57, 881, (1986).

105. N. J. Halas, J. Bokor, Phys. Rev. Lett, 62, 1679, (1989).

106. G. D. Kubiak, K. W. Kolasinski, J. Vac. Sci. A, 6, 814, (1988).

107. H. Eckhardt, L. Frische, J. Noffke, J. Phys. F: Met. Phys., 14, 97, (1984),

108. K. M. Ho, C. M. Fu, S. H. Liu, D. M. Kolb and G. Piazza, J. Electroanal. Chem, 150, 235, (1983).

109. P. O. Gartland and B. J. Slagsvold, Phys. Rev. B, 12, 4047, (1975).

110. S. D. Kevan and R. H. Gaylord, Phys. Rev. B, 36, 5809, (1987).

112. P.T. Coleridge and I. M. Templeton, Phys. Rev. B, 25, 7818 , (1982).

112. A. B. Chen and B. Segall, Sol. St. Com., 18, 149, (1976).

113. N.E. Christensen, phys. stat. sol. (b), 54, 551, (1972).

114. O. Jepsen, D. Glotzel and A. R. Mackintosh, Phys. Rev. B, 23, 2684, (1981). 
115. D. Liebowitz and N. J. Shevchik, Phys. Rev. B, 17, 3825, (1978).

126. P. S. Wehner, R. S. Williams, S. D. Kevan, D. Denley and D. A. Shirley, Phys. Rev. B, 19, 6164, (1979).

117. D. P. Spears, R. Melander, L. G. Petersson and S. B. M. Hagstrom, Phys. Rev. B, 21, 1462, (1980).

118. R. Courths, H. Wern, U. Hau, V. Bachelier and S. Hufner, J. Phys. F: Met. Phys., 14, 1559, (1984).

119. H. Ehrenreich and H. R. Phillipp, Phys. Rev., 128, 1622, (1.962).

1.20. B. Visscher and L.M. Falicov, phys. stat. sol. (b), 54, 9, (1972).

121. A. Gallo, PhD. Thesis, University of California, Berkeley, (1979).

122. K. Besocke and $s$. Berger, Rev. Sci. Inst., 47, 840, $(1976)$.

123. P. M. Whitmore, Iaboratory notebook entry, Feb. 20, 1981.

124. H. A. Engelhardt, D. Menzel, Sur. Sci, 57, 591, (1976).

125. B. Tsai, T. Baer and M. Horovitz, Rev. Sci. Inst., 45, $494,(1974)$.

126. R. 2. Bachrach, F. C. Brown and S. B. M. Hagstrom, J. Vac. Sci. Tech., 12, 309, (1975).

127. M. G. White, R. A. Rosenberg, G. Gabor, E. D. Poliakoff, G. Thornton, S. H. Southworth and D. A. Shirley, Rev. sci. Inst, 50, 1268, (1979).

128. J. Bokor, private commumication.

129. W. S. Fann, R. Storz and J. Bokor, Phys. Rev. B, 44, 10980, (1991).

130. R. Haight man, J. A. Silberman and M. I. Lilie, Rev. Sci. Inst., 59, 1941, (1988).

131. R. Haight and J. A. Silberman, Appl. Phys. Lett, 57, $1548,(1990)$

232, D. P. Shoemaker, C. W. Garland, J. I. Steinfeld, and J. W. Nibler, Experiments in Physical Chemistry, (McGraw Hill, 1981), p. 46. 
1.33. R. H. Fowler, Phys. Rev., 38, 45, (1931).

134. D. E. Eastman, J. J. Donelon, N. C. Hien and F. J. Himpsel, Nucl. Inst. and Meth., 172, 327, (1980)

135. N. V. Smith and S. D. Kevan, Nucl. Inst. and Meth., 195, 309 , (1982).

136. E. W. Plummer, Nucl. Inst. and Meth., 177, 179, (1980)

137. D. R. Lloyd, C. M. Quinn and N. V. Richardson, Sur. Sci., $689,547,(1977)$.

138. J. Unguris, L.W. Bruch, E. R. Moog and M. W. Webb, Sur. Sci., 87, 415, (1979).

139. J. Unguris, I.W. Bruch and M. W. Webb, Sur. Sci., 114, 219, (1982).

140. N. Schwentner, M. Skibowski and W. Steinmann, Phys. Rev. B, 8, 2965, (1973).

141. N. Schwentner, F. J. Himpsel, V. Saile, M. Skibowski, W. steinmann and E. E. Koch, Phys. Rev. Lett., 34, 528, (1975).

142. R. J. Behm, C. R. Brundle and K. Wandelt, J. Chem. Phys., 85, 1061, (1986).

143. A. J. Leadbetter and H. E.Thomas, Trans. Far. Soc., 61, 10, (1964).

144. J. E. Marcoux, J. Opt. Sci. Am., 59, 998, (1969).

145. I. E. Firment, G. A. Somorjai, J. Chem. Phys., 69, 3940, $(1978)$.

146. R. Kahn, R. Fourme, D. Andre and M. Renaud, Acta. Cryst., B29, 131, (1973).

147. A. E. Howard, S. W. Staley, Amer. Chem. Soc. Symp. Series, 263, 183, (1984). 

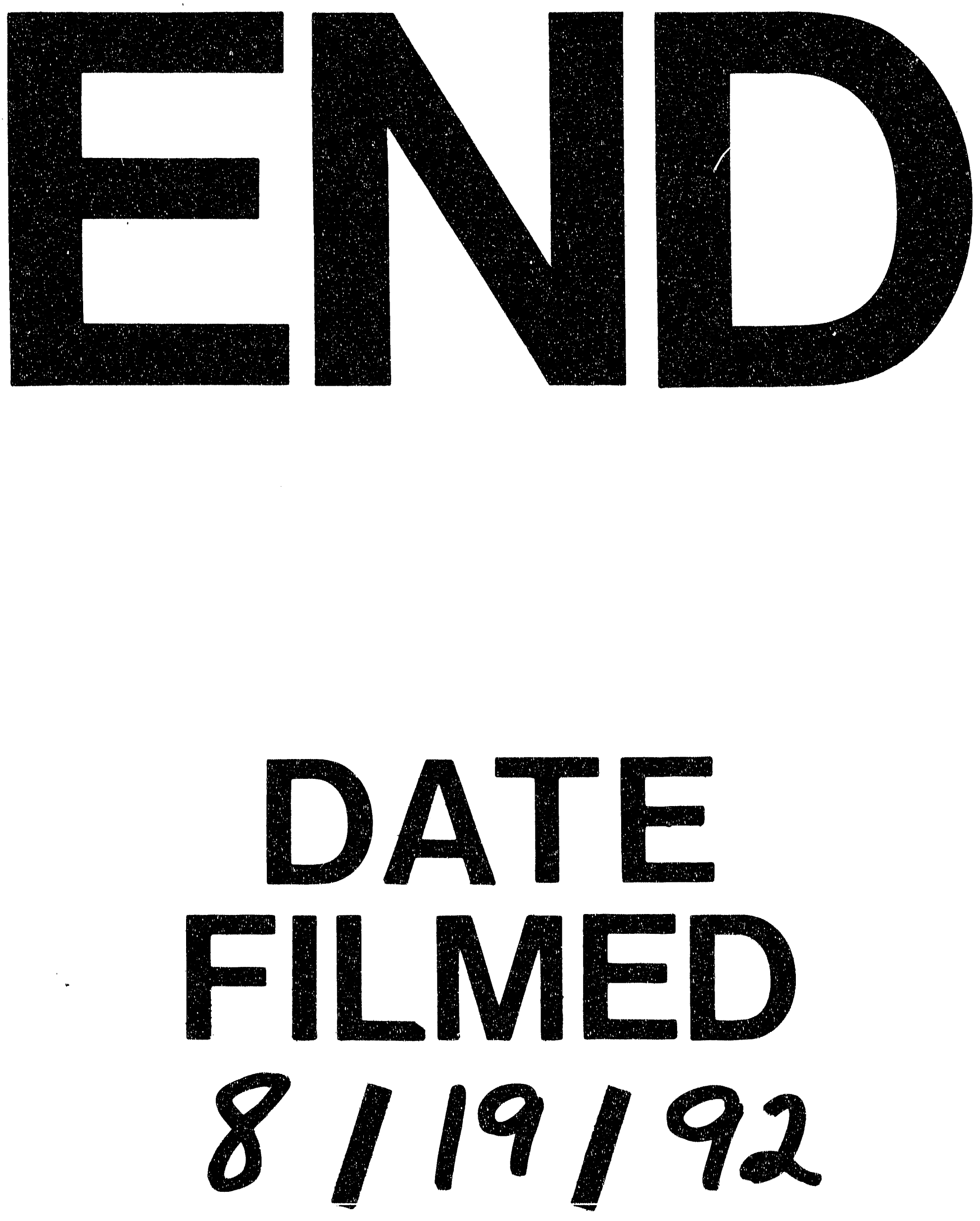


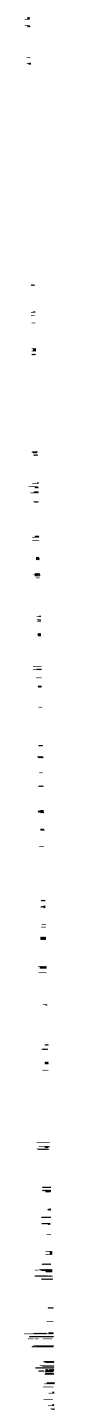

